\title{
THE TRACIAL ROKHLIN PROPERTY FOR ACTIONS OF FINITE GROUPS ON C*-ALGEBRAS
}

\author{
N. CHRISTOPHER PHILLIPS
}

\begin{abstract}
We define "tracial" analogs of the Rokhlin property for actions of finite groups, approximate representability of actions of finite abelian groups, and of approximate innerness. We prove the following four analogs of related "nontracial" results.

- The crossed product of an infinite dimensional simple separable unital $\mathrm{C}^{*}$-algebra with tracial rank zero by an action of a finite group with the tracial Rokhlin property again has tracial rank zero.

- An outer action of a finite abelian group on an infinite dimensional simple separable unital $\mathrm{C}^{*}$-algebra has the tracial Rokhlin property if and only if its dual is tracially approximately representable, and is tracially approximately representable if and only if its dual has the tracial Rokhlin property.

- If a strongly tracially approximately inner action of a finite cyclic group on an infinite dimensional simple separable unital $\mathrm{C}^{*}$-algebra has the tracial Rokhlin property, then it is tracially approximately representable.

- An automorphism of an infinite dimensional simple separable unital $\mathrm{C}^{*}$ algebra $A$ with tracial rank zero is tracially approximately inner if and only if it is the identity on $K_{0}(A)$ mod infinitesimals.
\end{abstract}

\section{INTRODUCTION}

Tracially $\mathrm{AF} \mathrm{C}^{*}$-algebras, now known as $\mathrm{C}^{*}$-algebras with tracial rank zero, were introduced in [14]. Roughly speaking, a $\mathrm{C}^{*}$-algebra has tracial rank zero if the local approximation characterization of AF algebras holds after cutting out a "small" approximately central projection. The term "tracial" comes from the fact that, in good cases, a projection $p$ is "small" if $\tau(p)<\varepsilon$ for every tracial state $\tau$ on $A$. The classification [17] of simple separable nuclear $\mathrm{C}^{*}$-algebras with tracial rank zero and satisfying the Universal Coefficient Theorem can be regarded as a vast generalization of the classification of $\mathrm{AF}$ algebras. This success suggests that one consider "tracial" versions of other $\mathrm{C}^{*}$-algebra concepts.

In this paper, motivated by applications to particular crossed products (see 23. and [7), we formulate and prove "tracial" versions of the following theorems:

- The crossed product of an AF algebra by an action of a finite group with the Rokhlin property is again AF (Theorem 2.2).

- An action of a finite abelian group on a unital $\mathrm{C}^{*}$-algebra has the Rokhlin property if and only if its dual is approximately representable, and is approximately representable if and only if its dual has the Rokhlin property. (Lemma 3.8 of [10]).

Date: 25 August 2006.

2000 Mathematics Subject Classification. Primary 46L55; Secondary 46L40.

Research partially supported by NSF grants DMS 0070776 and DMS 0302401. 
- If an approximately inner action of a finite cyclic group on a unital $\mathrm{C}^{*}$ algebra has the Rokhlin property, then it is approximately representable (Proposition 4.11).

- An automorphism of an AF algebra $A$ is approximately inner if and only if it is the identity on $K_{0}(A)$ (part of Theorem 3.1 of [2]).

Our results are:

- The crossed product of an infinite dimensional simple separable unital $\mathrm{C}^{*}$ algebra with tracial rank zero by an action of a finite group with the tracial Rokhlin property again has tracial rank zero (Theorem 2.6).

- An outer action of a finite abelian group on an infinite dimensional simple separable unital $\mathrm{C}^{*}$-algebra has the tracial Rokhlin property if and only if its dual is tracially approximately representable, and is tracially approximately representable if and only if its dual has the tracial Rokhlin property (Theorem 3.11).

- If a strongly tracially approximately inner action of a finite cyclic group on an infinite dimensional simple separable unital $\mathrm{C}^{*}$-algebra has the tracial Rokhlin property, then it is tracially approximately representable (Theorem 4.6).

- An automorphism of an infinite dimensional simple separable unital $\mathrm{C}^{*}$ algebra $A$ with tracial rank zero is tracially approximately inner if and only if it is the identity on $K_{0}(A)$ mod infinitesimals (Theorem 6.4).

The first three of these results were chosen because they are used in the proof 23. that every simple higher dimensional noncommutative torus is an AT algebra. (We have not found the "nontracial" versions of the first and third results in the literature. Therefore they are also proved in this paper.) The last is related to our effort to find the "right" definition of a tracially approximately inner automorphism. Annoyingly, the strongly tracially approximately inner automorphisms (as in the third result) probably don't form a group. On the other hand, an automorphism of an infinite dimensional simple separable unital $\mathrm{C}^{*}$-algebra with tracial rank zero which is tracially approximately inner and has finite order must in fact be strongly tracially approximately inner. (Combine Proposition 6.2 and Theorem 6.6])

In retrospect, the following motivation is perhaps better. In [10] and [1], Izumi has started an intensive study of finite group actions with the Rokhlin property, which, to minimize confusion, we call here the strict Rokhlin property. The strict Rokhlin property imposes severe restrictions on the relation between the K-theory of the original algebra, the action of the group on this K-theory, and the K-theory of the crossed product. See especially Section 3 of [11. Since actions with the strict Rokhlin property are so rare, a less restrictive version of the Rokhlin property is needed. We give some examples; in them, we write $\mathbb{Z}_{n}$ for $\mathbb{Z} / n \mathbb{Z}$.

- The flip action of $\mathbb{Z}_{2}$ on $A \otimes A$, for an infinite dimensional simple separable unital $\mathrm{C}^{*}$-algebra $A$, often has the tracial Rokhlin property, but probably almost never has the strict Rokhlin property. See [21].

- Let $A$ be a simple higher dimensional noncommutative torus, with standard unitary generators $u_{1}, u_{2}, \ldots, u_{d}$. Consider the automorphism which sends $u_{k}$ to $\exp (2 \pi i / n) u_{k}$, and fixes $u_{j}$ for $j \neq k$. This automorphism generates an action of $\mathbb{Z}_{n}$ which has the tracial Rokhlin property, but for $n>1$ never has strict Rokhlin property. The fact that this action has the tracial 
Rokhlin property plays a key role in the classification [23] of simple higher dimensional noncommutative toruses.

- Again let $A$ be a simple higher dimensional noncommutative torus, with standard unitary generators $u_{1}, u_{2}, \ldots, u_{d}$. The flip automorphism $u_{k} \mapsto u_{k}^{*}$ generates an action of $\mathbb{Z}_{2}$ which has the tracial Rokhlin property, but never has the strict Rokhlin property. See [7], where this fact is used to prove that the crossed product by the flip action is always AF.

- The standard actions of $\mathbb{Z}_{3}, \mathbb{Z}_{4}$, and $\mathbb{Z}_{6}$ on an irrational rotation algebra all have the tracial Rokhlin property, but never have the strict Rokhlin property. In [7, this is used to prove that the crossed products are always $\mathrm{AF}$ algebras.

Of course, one can't expect classification results for actions of the kind found in [10] and [11.

This paper is devoted to the general theory. In 24, we give several useful criteria for the tracial Rokhlin property, and we give a number of examples of actions of $\mathbb{Z}_{2}$ on $\mathrm{C}^{*}$-algebras with tracial rank zero (mostly AF algebras) which do and do not have the tracial Rokhlin property, and are or are not tracially approximately representable. Further examples, and results for $\mathrm{C}^{*}$-algebras with finite but nonzero tracial rank, will appear in [21]. The main applications, already mentioned above, are in [23] and [7. We also point out that the tracial Rokhlin property has a generalization to integer actions, considered in [19] and with applications given in [20]. Presumably there is a useful generalization to other countable amenable groups.

This paper replaces Sections 1 through 4 and Section 11 of the unpublished long preprint [22]. The material of Sections 5 through 7 there will appear in 23, a greatly improved version of Sections 8 through 10 will appear in [7, and an improved and expanded version of the material in Sections 12 and 13 will appear in 24. We give the theory for actions of finite groups, or of finite abelian groups, as appropriate; in 22, only finite cyclic groups were considered. The definition of the tracial Rokhlin property given here differs slightly from that in [22]; see Remark 1.3 and the following discussion for details. There is no difference for actions on simple unital $\mathrm{C}^{*}$-algebras with tracial rank zero. The definition of tracial approximate innerness (Definition 5.1) has been greatly improved; more automorphisms satisfy the condition than satisfied the condition in [22], and with the new definition the tracially approximately inner automorphisms form a group. (See Theorem [5.8) A variant of the original definition appears in Definition 4.2 here, where the condition is called strong tracial approximate innerness. The material in Section 3 on tracial approximate representability is mostly new, although it was motivated by one of the key results in [22] and its parallel with Lemma 3.8 of [10].

This paper is organized as follows. In Section 1 we introduce the tracial Rokhlin property and prove some basic properties. Some of the lemmas will be used repeatedly in connection with the tracial versions of other properties. Section 2 contains the proofs that the crossed product of an AF algebra by an action of a finite group with the Rokhlin property is again AF, and that the crossed product of an infinite dimensional simple separable unital $\mathrm{C}^{*}$-algebra with tracial rank zero by an action of a finite group with the tracial Rokhlin property again has tracial rank zero. In Section 3 we treat tracial approximate representability for actions of finite abelian groups, and prove the duality between this property and the tracial 
Rokhlin property, corresponding to Lemma 3.8 of [10]. In Section 4 we introduce strongly tracially approximately inner automorphism, and prove that if a strongly tracially approximately inner action of a finite cyclic group on an infinite dimensional simple separable unital $\mathrm{C}^{*}$-algebra has the tracial Rokhlin property, then it is tracially approximately representable. We also prove the "nontracial" analog of this result. Sections [5 and 6] treat tracially approximately inner automorphisms. We show that they form a group. We prove that they act trivially on the tracial state space and on $K_{0}$ mod infinitesimals, and give a converse when the algebra is simple with tracial rank zero. We also prove that if the the algebra is simple with tracial rank zero, then a tracially approximately inner automorphism of finite order is necessarily strongly tracially approximately inner.

We use the following notation. We write $p \precsim q$ to mean that the projection $p$ is Murray-von Neumann equivalent to a subprojection of $q$, and $p \sim q$ to mean that $p$ is Murray-von Neumann equivalent to $q$. Also, $[a, b]$ denotes the additive commutator $a b-b a$. If $A$ is a $\mathrm{C}^{*}$-algebra and $\alpha: G \rightarrow \operatorname{Aut}(A)$ is a group action, we write $A^{\alpha}$ for the fixed point algebra.

In most arguments dealing with an arbitrary finite subset $F$ of a $\mathrm{C}^{*}$-algebra, we will normalize and assume that all elements of $F$ have norm at most 1.

We are grateful to Hanfeng Li for valuable comments, and to Hiroyuki Osaka for a careful reading and suggesting improvements to several of the proofs. We are also grateful to Dawn Ashley for catching a number of misprints and minor mistakes.

\section{The TRACIAL RokHLin PROPERTY}

In this section we introduce the tracial Rokhlin property. We observe several elementary relations and consequences, and we prove several useful equivalent formulations. Some of the technical lemmas will be repeatedly used in connection with other "tracial" properties.

We begin with Izumi's definition of the Rokhlin property. To emphasize the difference, we call it the strict Rokhlin property here.

Definition 1.1. Let $A$ be a separable unital $\mathrm{C}^{*}$-algebra, and let $\alpha: G \rightarrow \operatorname{Aut}(A)$ be an action of a finite group $G$ on $A$. We say that $\alpha$ has the strict Rokhlin property if for every finite set $F \subset A$, and every $\varepsilon>0$, there are mutually orthogonal projections $e_{g} \in A$ for $g \in G$ such that:

(1) $\left\|\alpha_{g}\left(e_{h}\right)-e_{g h}\right\|<\varepsilon$ for all $g, h \in G$.

(2) $\left\|e_{g} a-a e_{g}\right\|<\varepsilon$ for all $g \in G$ and all $a \in F$.

(3) $\sum_{g \in G} e_{g}=1$.

Izumi's definition (Definition 3.1 of [10]) is actually in terms of central sequences. Thus, it yields not mutually orthogonal projections but elements $b_{g} \in A$ such that $\left\|b_{g} b_{h}-\delta_{g, h} b_{g}\right\|<\varepsilon$ for $g, h \in G$, such that $\left\|b_{g}^{*}-b_{g}\right\|<\varepsilon$ for $g \in G$, and such that $\left\|1-\sum_{g \in G} b_{g}\right\|<\varepsilon$. However, with $n=\operatorname{card}(G)$, using semiprojectivity of $\mathbb{C}^{n}$ (see Lemma 14.1.5, Theorem 14.2.1, Theorem 14.1.4, and Definition 14.1.1 of [18]) and a suitably smaller choice of $\varepsilon$, one easily sees that the definition above is equivalent to Definition 3.1 of [10.

If $\alpha$ is approximately inner, requiring $\sum_{g \in G} e_{g}=1$ forces $\left[1_{A}\right] \in K_{0}(A)$ to be divisible by the order of $G$, and therefore rules out many $\mathrm{C}^{*}$-algebras of interest. In fact, the strict Rokhlin property imposes much more stringent conditions on the 
K-theory. Theorem 3.3 and Lemma 3.2(1) of [1] show that if a nontrivial finite group $G$ acts on a simple unital $\mathrm{C}^{*}$-algebra $A$ in such a way that the induced action on $K_{*}(A)$ is trivial, and if one of $K_{0}(A)$ and $K_{1}(A)$ is a nonzero free abelian group, then $\alpha$ does not have the strict Rokhlin property. Theorem 3.3 and the discussion preceding Theorem 3.4 of [11] show that if in addition $G$ is cyclic of order $n$, then the strict Rokhlin property implies that $K_{*}(A)$ is uniquely $n$-divisible. It follows that the actions considered in our main applications (to simple higher dimensional noncommutative toruses [23] and irrational rotation algebras [7) never have the strict Rokhlin property.

We now give the definition of the tracial Rokhlin property. The difference is that we do not require that $\sum_{g \in G} e_{g}=1$, only that $1-\sum_{g \in G} e_{g}$ be "small" in a tracial sense. Of course, $\sum_{g \in G} e_{g}=1$ is allowed, in which case Conditions (36) and (4) in the definition are vacuous.

Definition 1.2. Let $A$ be an infinite dimensional simple separable unital $\mathrm{C}^{*}$ algebra, and let $\alpha: G \rightarrow \operatorname{Aut}(A)$ be an action of a finite group $G$ on $A$. We say that $\alpha$ has the tracial Rokhlin property if for every finite set $F \subset A$, every $\varepsilon>0$, and every positive element $x \in A$ with $\|x\|=1$, there are mutually orthogonal projections $e_{g} \in A$ for $g \in G$ such that:

(1) $\left\|\alpha_{g}\left(e_{h}\right)-e_{g h}\right\|<\varepsilon$ for all $g, h \in G$.

(2) $\left\|e_{g} a-a e_{g}\right\|<\varepsilon$ for all $g \in G$ and all $a \in F$.

(3) With $e=\sum_{g \in G} e_{g}$, the projection $1-e$ is Murray-von Neumann equivalent to a projection in the hereditary subalgebra of $A$ generated by $x$.

(4) With $e$ as in (3), we have $\|$ exe $\|>1-\varepsilon$.

Remark 1.3. Our original definition, in 22], in addition specified a positive integer $N$, and required the following condition instead of (4):

$\left(4^{\prime}\right)$ For every $g \in G$, there are $N$ mutually orthogonal projections $f_{1}, f_{2}, \ldots, f_{N} \leq$ $e_{j}$, each of which is Murray-von Neumann equivalent to the projection $1-e$ of (3).

As we will see in Lemma 1.16 below, when $A$ is finite, Condition (4) in Definition 1.2 is unnecessary. When $A$ has finite tracial topological rank in the sense of [15], it is not hard to see that Definition 1.2 implies the definition in Remark 1.3. In general, the situation is less clear, and it might be necessary to use both Condition (4) and Condition $\left(4^{\prime}\right)$, especially for nonsimple $\mathrm{C}^{*}$-algebras. We postpone further discussion to Section 4 of [24].

Remark 1.4. If an action of a finite group $G$ on an infinite dimensional simple separable unital $\mathrm{C}^{*}$-algebra $A$ has the strict Rokhlin property, then it has the tracial Rokhlin property.

Lemma 1.5. Let $A$ be an infinite dimensional simple separable unital $\mathrm{C}^{*}$-algebra, and let $\alpha: G \rightarrow \operatorname{Aut}(A)$ be an action of a finite group $G$ on $A$ which has the tracial Rokhlin property. Then $\alpha_{g}$ is outer for every $g \in G \backslash\{1\}$.

Proof. Let $g \in G \backslash\{1\}$ and let $u \in A$ be unitary. We prove that $\alpha_{g} \neq \operatorname{Ad}(u)$. Apply Definition 1.2 with $F=\{u\}$, with $\varepsilon=\frac{1}{2}$, and with $x=1$. Then $e_{1}$ and $e_{g}$ are orthogonal nonzero projections, so

$$
\left\|\alpha_{g}\left(e_{1}\right)-u e_{1} u^{*}\right\| \geq\left\|e_{g}-e_{1}\right\|-\left\|\alpha_{g}\left(e_{1}\right)-e_{g}\right\|-\left\|u e_{1} u^{*}-e_{1}\right\|>0 .
$$

Therefore $\alpha_{g} \neq \operatorname{Ad}(u)$. 
Corollary 1.6. Let $A$ be an infinite dimensional simple separable unital $\mathrm{C}^{*}$ algebra, and let $\alpha: G \rightarrow \operatorname{Aut}(A)$ be an action of a finite group $G$ on $A$ which has the tracial Rokhlin property. Then $C^{*}(G, A, \alpha)$ is simple.

Proof. In view of Lemma 1.5 this follows from Theorem 3.1 of [13].

For the tracial Rokhlin property to be likely to hold, the $\mathrm{C}^{*}$-algebra must have a reasonable number of projections. For reference, we recall here the definition of the property that seems most relevant.

Definition 1.7. Let $A$ be a $\mathrm{C}^{*}$-algebra. We say that $A$ has Property $(S P)$ if every nonzero hereditary subalgebra in $A$ contains a nonzero projection.

We state here some results about simple $\mathrm{C}^{*}$-algebras with Property (SP) that will be used repeatedly in this paper.

Lemma 1.8. Let $A$ be a $\mathrm{C}^{*}$-algebra, and let $c \in A$. Then for any projection $p \in \overline{c A c^{*}}$, there exists a projection $q \in \overline{c^{*} A c}$ such that $p \sim q$.

Proof. This is essentially in Section 1 of [5]. The details can be found in the proof of Lemma 4.1 of [19].

The following lemma is essentially Lemma 3.1 of [14, but no proof is given there.

Lemma 1.9. Let $A$ be a simple $\mathrm{C}^{*}$-algebra with Property (SP). Let $B \subset A$ be a nonzero hereditary subalgebra, and let $p \in A$ be a nonzero projection. Then there is a nonzero projection $q \in B$ such that $q \precsim p$.

Proof. Choose a nonzero positive element $a \in B$. Since $A$ is simple, there exists $x \in A$ such that $c=\operatorname{axp}$ is nonzero. Choose a nonzero projection $q \in \overline{c A c^{*}}$. Then $q \in B$, and Lemma 4.1 of [19] (or Lemma [1.8) provides a projection $e \leq p$ such that $q \sim e$.

Lemma 1.10. Let $A$ be an infinite dimensional simple unital $\mathrm{C}^{*}$-algebra with Property (SP). Let $B \subset A$ be a nonzero hereditary subalgebra, and let $n \in \mathbb{N}$. Then there exist nonzero Murray-von Neumann equivalent mutually orthogonal projections $p_{1}, p_{2}, \ldots, p_{n} \in B$.

Proof. Since $A$ is unital and infinite dimensional, it is not isomorphic to the algebra of compact operators on any Hilbert space. The lemma is then immediate from Lemma 3.2 of 14. (The result from [1] is on page 61 of that reference. See above for the proof of Lemma 3.1 of [14.)

Lemma 1.11. Let $A$ be an infinite dimensional simple unital $\mathrm{C}^{*}$-algebra, and let $n \in \mathbb{N}$. Then $A$ has Property (SP) if and only if $M_{n} \otimes A$ has Property (SP). Moreover, in this case, for every nonzero hereditary subalgebra $B \subset M_{n} \otimes A$, there exists a nonzero projection $p \in A$ such that $1 \otimes p$ is Murray-von Neumann equivalent to a projection in $B$.

Proof. Since $A$ is isomorphic to a hereditary subalgebra in $M_{n} \otimes A$, it is obvious that if $M_{n} \otimes A$ has Property (SP), then so does $A$.

For the converse and the last statement, choose a nonzero element $x \in B$. Let $\left(e_{j, k}\right)_{1 \leq j, k \leq n}$ be a system of matrix units for $M_{n}$. Choose $j$ such that $\left(e_{j, j} \otimes 1\right) x \neq 0$. Then $C=\left(e_{j, j} \otimes 1\right) x\left(M_{n} \otimes A\right) x^{*}\left(e_{j, j} \otimes 1\right)$ is a nonzero hereditary subalgebra in $\left(e_{j, j} \otimes 1\right)\left(M_{n} \otimes A\right)\left(e_{j, j} \otimes 1\right) \cong A$. Because $A$ has Property (SP), there exists a nonzero 
projection $f \in A$ such that $e_{j, j} \otimes f \in C$. By Lemma 1.10 there exist nonzero Murray-von Neumann equivalent mutually orthogonal projections $f_{1}, f_{2}, \ldots, f_{n} \in$ $A$ with $f_{j} \leq f$ for all $k$. Then

$$
1 \otimes f_{1}=\sum_{k=1}^{n} e_{k, k} \otimes f_{1} \sim \sum_{k=1}^{n} e_{j, j} \otimes f_{k} \leq e_{j, j} \otimes f
$$

and $e_{j, j} \otimes f$ is Murray-von Neumann equivalent to a projection in $B$ by Lemma 1.8

The following result implies, in particular, that if $A$ is an infinite dimensional simple unital $\mathrm{C}^{*}$-algebra with Property (SP), and if $\alpha: G \rightarrow \operatorname{Aut}(A)$ is an action of a finite group $G$ on $A$ such that the crossed product is also simple, then the crossed product has Property (SP).

Proposition 1.12. Let $A$ be an infinite dimensional simple unital $\mathrm{C}^{*}$-algebra with Property (SP), and let $\alpha: G \rightarrow \operatorname{Aut}(A)$ be an action of a finite group $G$ on $A$ such that $C^{*}(G, A, \alpha)$ is also simple. Let $B \subset C^{*}(G, A, \alpha)$ be a nonzero hereditary subalgebra. Then there exists a nonzero projection $p \in A$ which is Murray-von Neumann equivalent in $C^{*}(G, A, \alpha)$ to a projection in $B$.

Proof. Set $N=\left\{g \in G: \alpha_{g}\right.$ is inner $\}$. Theorem 4.2 of [12] provides a projection $q \in C^{*}\left(N, A,\left.\alpha\right|_{N}\right) \subset C^{*}(G, A, \alpha)$ which is Murray-von Neumann equivalent in $C^{*}(G, A, \alpha)$ to a projection in $B$. (There is a reference missing in its proof: one uses the discussion after Proposition 2.8 of 25] for the claim, at the top of page 295 of [12], that $C^{*}\left(N, A,\left.\alpha\right|_{N}\right)$ is a direct sum of matrix algebras over $A$.) Let $G$ act on $C^{*}\left(N, A,\left.\alpha\right|_{N}\right)$ by conjugation by the standard unitaries $u_{g} \in C^{*}(G, A, \alpha)$, as in the discussion before Proposition 2.4 of [25]. (Since $A$ is simple and unital, an automorphism is partly inner in the sense of [25] if and only if it is inner.) Following the discussion after Proposition 2.8 of $\left[25\right.$, there is a finite dimensional $\mathrm{C}^{*}$-algebra $C$ with an action $\gamma$ of $G$ such that $C^{*}\left(N, A,\left.\alpha\right|_{N}\right)$ is equivariantly isomorphic to $A \otimes C$ with the action $g \mapsto \alpha_{g} \otimes \gamma_{g}$, in such a way that the inclusion of $A$ in $C^{*}\left(N, A,\left.\alpha\right|_{N}\right)$ becomes $a \mapsto a \otimes 1$. Moreover, $C$ is $G$-simple by Propositions 2.5 and 2.10 of [25]. Let $e_{1}, e_{2}, \ldots, e_{n}$ be the minimal central projections of $C$. There is some $k$ such that the projection $\left(1 \otimes e_{k}\right) q$ is nonzero. Lemma1.11 provides a nonzero projection $f \in A$ such that $f \otimes e_{k} \precsim\left(1 \otimes e_{k}\right) q$. By Lemma1.10 there exist nonzero Murray-von Neumann equivalent mutually orthogonal projections $f_{1}, f_{2}, \ldots, f_{n} \in A$ with $f_{j} \leq f$ for all $j$. Since $C$ is $G$-simple, there exist $g_{1}, g_{2}, \ldots, g_{n} \in G$ such that $\gamma_{g_{j}}\left(e_{j}\right)=e_{k}$ for $1 \leq j \leq n$. Use Lemma 1.9 repeatedly to find a projection $p \in A$ such that $p \precsim \alpha_{g_{j}}^{-1}\left(f_{j}\right)$ for all $j$. Then, with the first relation holding in $C^{*}(G, A, \alpha)$ and the second in $C^{*}\left(N, A,\left.\alpha\right|_{N}\right)=A \otimes C$, we have

$$
p \otimes e_{j} \sim \alpha_{g_{j}}(p) \otimes e_{k} \precsim f_{j} \otimes e_{k} .
$$

It follows that, in $C^{*}(G, A, \alpha)$, we have

$$
p \otimes 1 \precsim \sum_{j=1}^{n} f_{j} \otimes e_{k} \leq f \otimes e_{k} \precsim\left(1 \otimes e_{k}\right) q \leq q .
$$

Since $q$ is Murray-von Neumann equivalent in $C^{*}(G, A, \alpha)$ to a projection in $B$, the proof is complete.

We now return to the main development. 
Lemma 1.13. Let $A$ be an infinite dimensional simple separable unital $\mathrm{C}^{*}$-algebra, and let $\alpha: G \rightarrow \operatorname{Aut}(A)$ be an action of a finite group $G$ on $A$ which has the tracial Rokhlin property. Then $A$ has Property (SP) or $\alpha$ has the strict Rokhlin property.

Proof. If $A$ does not have Property (SP), then there is a nonzero positive element $x \in A$ which generates a hereditary subalgebra which contains no nonzero projection.

The following two lemmas will be important for dealing with Condition (4) of Definition 1.2 and with similar conditions in other definitions in this paper. The first comes from an argument that goes back to Cuntz, in the proof of Lemma 1.7 of [6].

Lemma 1.14. Let $A$ be a $\mathrm{C}^{*}$-algebra with Property (SP), let $x \in A$ be a positive element with $\|x\|=1$, and let $\varepsilon>0$. Then there exists a nonzero projection $p \in \overline{x A x}$ such that, whenever $q \leq p$ is a nonzero projection, then

$$
\|q x q-q\|<\varepsilon, \quad\|q x-x q\|<\varepsilon, \quad \text { and } \quad\|q x q\|>1-\varepsilon .
$$

Proof. Choose continuous functions $h_{1}, h_{2}:[0,1] \rightarrow[0,1]$ such that $h_{1}(0)=0$, $h_{1}(t)=1$ for $t \geq 1-\frac{1}{4} \varepsilon$, and $\left|h_{1}(t)-t\right| \leq \frac{1}{4} \varepsilon$ for all $t$, and such that $h_{2}(1)=1$ and $h_{1} h_{2}=h_{2}$. Set $y=h_{1}(x)$ and $z=h_{2}(x)$. Then $\|x-y\| \leq \frac{1}{4} \varepsilon$ and $y z=z$. Furthermore, $z \neq 0$ because $1 \in \operatorname{sp}(x)$. By Property (SP), there is a nonzero projection $p \in \overline{z A z}$. Let $q \leq p$ be a nonzero projection. Then $q \in \overline{z A z}$, so $y q=$ $q y=q$. Therefore

$$
\|q x-x q\| \leq 2\|x-y\| \leq \frac{1}{2} \varepsilon<\varepsilon .
$$

Furthermore,

$$
\|q x q-q\|=\|q x q-q y q\| \leq\|x-y\| \leq \frac{1}{4} \varepsilon<\varepsilon,
$$

whence also $\|q x q\|>1-\varepsilon$.

Lemma 1.15. Let $A$ be an infinite dimensional finite unital $\mathrm{C}^{*}$-algebra with Property (SP), let $x \in A$ be a positive element with $\|x\|=1$, and let $\varepsilon>0$. Then there exists a nonzero projection $q \in \overline{x A x}$ such that, whenever $e \in A$ is a projection such that $1-e \precsim q$, then $\|$ exe $\|>1-\varepsilon$.

Proof. Apply Lemma 1.14 with $x^{1 / 2}$ in place of $x$ and with $\frac{1}{5} \varepsilon$ in place of $\varepsilon$, obtaining a nonzero projection $p \in \overline{x^{1 / 2} A x^{1 / 2}}$ such that, whenever $q \leq p$ is a nonzero projection, then (combining two of the estimates there) $\left\|q x^{1 / 2}-q\right\|<\frac{2}{5} \varepsilon$. Note that $\overline{x^{1 / 2} A x^{1 / 2}}=\overline{x A x}$. By Lemma 1.10 there is a nonzero projection $q \leq p$ such that $p-q \neq 0$. Now suppose $e \in A$ is a projection such that $1-e \precsim q$ and $\|e x e\| \leq 1-\varepsilon$. Then, repeatedly using $\left\|a^{*} a\right\|=\left\|a a^{*}\right\|$, we get

$$
\| \text { epe }\|=\| p e p\|<\| p x^{1 / 2} e x^{1 / 2} p\left\|+\frac{4}{5} \varepsilon \leq\right\| x^{1 / 2} e x^{1 / 2}\left\|+\frac{4}{5} \varepsilon=\right\| e x e \|+\frac{4}{5} \varepsilon<1-\frac{1}{5} \varepsilon .
$$

Therefore

$$
\|e-e(1-p)\|=\|e p\|=\|e p e\|^{1 / 2}<\left(1-\frac{1}{5} \varepsilon\right)^{1 / 2}<1 .
$$

It follows from Lemma 2.5.2 of [16] that $e \precsim 1-p$. Since, by assumption, we have $1-e \precsim q$, this gives $1 \precsim 1-(p-q)$, contradicting finiteness of $A$.

When $A$ is finite, we do not need Condition (4) of Definition 1.2 
Lemma 1.16. Let $A$ be an infinite dimensional finite simple separable unital $\mathrm{C}^{*}$ algebra, and let $\alpha: G \rightarrow \operatorname{Aut}(A)$ be an action of a finite group $G$ on $A$. Then $\alpha$ has the tracial Rokhlin property if and only if for every finite set $F \subset A$, every $\varepsilon>0$, and every nonzero positive element $x \in A$, there are mutually orthogonal projections $e_{g} \in A$ for $g \in G$ such that:

(1) $\left\|\alpha_{g}\left(e_{h}\right)-e_{g h}\right\|<\varepsilon$ for all $g, h \in G$.

(2) $\left\|e_{g} a-a e_{g}\right\|<\varepsilon$ for all $g \in G$ and all $a \in F$.

(3) With $e=\sum_{g \in G} e_{g}$, the projection $1-e$ is Murray-von Neumann equivalent to a projection in the hereditary subalgebra of $A$ generated by $x$.

Proof. It is immediate that the tracial Rokhlin property implies the condition in the lemma. So assume the condition in the lemma holds.

If $A$ does not have Property (SP), then $\alpha$ has the strict Rokhlin property, by the same proof as for Lemma 1.13 Accordingly, we may assume that $A$ has Property (SP).

Let $F \subset A$ be finite, let $\varepsilon>0$, and let $x \in A$ be a positive element with $\|x\|=1$. Apply Lemma 1.15 obtaining a nonzero projection $q \in \overline{x A x}$ such that, whenever $e \in A$ is a projection such that $1-e \precsim q$, then $\|e x e\|>1-\varepsilon$. Apply the hypothesis, with $F$ and $\varepsilon$ as given, and with $q$ in place of $x$, obtaining projections $e_{g} \in A$ for $g \in G$. Set $e=\sum_{g \in G} e_{g}$. We need only prove that $\|$ exe $\|>1-\varepsilon$. But this is immediate from the choice of $q$ and the relation $1-e \precsim q$.

It is convenient to have a formally stronger version of the tracial Rokhlin property, in which the defect projection is $\alpha$-invariant.

Lemma 1.17. Let $A$ be an infinite dimensional simple separable unital $\mathrm{C}^{*}$-algebra, and let $\alpha: G \rightarrow \operatorname{Aut}(A)$ be an action of a finite group $G$ on $A$ which has the tracial Rokhlin property. Let $F \subset A$ be finite, let $\varepsilon>0$, and let $x \in A$ be a positive element with $\|x\|=1$. Then there are mutually orthogonal projections $e_{g} \in A$ for $g \in G$ such that:

(1) $\left\|\alpha_{g}\left(e_{h}\right)-e_{g h}\right\|<\varepsilon$ for all $g, h \in G$.

(2) $\left\|e_{g} a-a e_{g}\right\|<\varepsilon$ for all $g \in G$ and all $a \in F$.

(3) The projection $e=\sum_{g \in G} e_{g}$ is $\alpha$-invariant.

(4) With $e$ as in (3), the projection $1-e$ is Murray-von Neumann equivalent to a projection in the hereditary subalgebra of $A$ generated by $x$.

(5) With $e$ as in (3), we have $\|$ exe $\|>1-\varepsilon$.

Proof. Without loss of generality $\|a\| \leq 1$ for all $a \in F$. Set $\varepsilon_{0}=\min \left(\frac{1}{5} \varepsilon, \frac{1}{2}\right)$. Choose $\delta>0$ so small that whenever $B$ is a unital $\mathrm{C}^{*}$-algebra, $b \in B$ is selfadjoint, and $p \in B$ is a projection such that $\|b-p\|<\operatorname{card}(G) \delta$, then the functional calculus $e=\chi_{(1 / 2, \infty)}(b)$ is defined and moreover $\|e-p\|$ is small enough that there exists a unitary $v \in B$ such that $v p v^{*}=e$ and $\|v-1\|<\varepsilon_{0}$. We also require $\delta \leq \varepsilon_{0}$.

Apply Definition 1.2 to $\alpha$, with $F$ and $x$ as given, and with $\delta$ in place of $\varepsilon$. obtaining projections $e_{g} \in A$ for $g \in G$. Let $\left(p_{g}\right)_{g \in G}$ be the resulting family of projections. Define $p=\sum_{h \in G} p_{h}$. For $g \in G$ we have

$$
\left\|\alpha_{g}(p)-p\right\| \leq \sum_{h \in G}\left\|\alpha_{g}\left(p_{h}\right)-p_{g h}\right\|<\operatorname{card}(G) \delta .
$$

Set

$$
b=\frac{1}{\operatorname{card}(G)} \sum_{g \in G} \alpha_{g}(p) .
$$


Then $b \in A^{\alpha}$ and $\|b-p\|<\operatorname{card}(G) \delta$.

By the choice of $\delta$, there exists a projection $e \in A^{\alpha}$ and a unitary $v \in A$ such that $v p v^{*}=e$ and $\|v-1\|<\varepsilon_{0}$. Now define $e_{g}=v p_{g} v^{*}$ for $g \in G$. Clearly $\left\|e_{g}-p_{g}\right\|<2 \varepsilon_{0}$. So, for $g, h \in G$,

$$
\left\|\alpha_{g}\left(e_{h}\right)-e_{g h}\right\| \leq\left\|e_{h}-p_{h}\right\|+\left\|e_{g h}-p_{g h}\right\|+\left\|\alpha_{g}\left(p_{h}\right)-p_{g h}\right\|<5 \varepsilon_{0} \leq \varepsilon .
$$

For $g \in G$ and $a \in F$, we similarly get $\left\|e_{g} a-a e_{g}\right\|<5 \varepsilon_{0} \leq \varepsilon$. We have

$$
\|(1-e)-(1-p)\|<2 \varepsilon_{0} \leq 1
$$

so $1-e \sim 1-p$, and is hence Murray-von Neumann equivalent to a projection in the hereditary subalgebra of $A$ generated by $x$. Finally,

$$
\|\operatorname{exe}\| \geq\|p x p\|-2\|e-p\|>1-\delta-2 \varepsilon_{0} \geq 1-\varepsilon .
$$

This completes the proof.

\section{Crossed products by aCtions on $\mathrm{C}^{*}$-Algebras with tracial Rank ZERO}

The main result of this section is that the crossed product of an infinite dimensional simple separable unital $\mathrm{C}^{*}$-algebra with tracial rank zero by an action with the tracial Rokhlin property again has tracial rank zero. We begin by proving the analogous result in which the word "tracial" is omitted everywhere: the crossed product of a unital AF -algebra by an action with the strict Rokhlin property is again AF. To our surprise, we have been unable to find this result in the literature. Both proofs have been considerably simplified from original versions, following a suggestion of Osaka. The main estimates in the proof for actions with the strict Rokhlin property will be referred to in the proof for actions with the tracial Rokhlin property. Example 3.1 of 24] shows that the hypotheses can't be weakened to require only the tracial Rokhlin property.

We need a lemma.

Lemma 2.1. Let $n \in \mathbb{N}$. For every $\varepsilon>0$ there is $\delta>0$ such that, whenever $\left(e_{j, k}\right)_{1 \leq j, k \leq n}$ is a system of matrix units for $M_{n}$, whenever $B$ is a unital $\mathrm{C}^{*}$-algebra, and whenever $w_{j, k}$, for $1 \leq j, k \leq n$, are elements of $B$ such that $\left\|w_{j, k}^{*}-w_{k, j}\right\|<\delta$ for $1 \leq j, k \leq n$, such that $\left\|w_{j_{1}, k_{1}} w_{j_{2}, k_{2}}-\delta_{j_{2}, k_{1}} w_{j_{1}, k_{2}}\right\|<\delta$ for $1 \leq j_{1}, j_{2}, k_{1}, k_{2} \leq n$, and such that the $w_{j, j}$ are orthogonal projections with $\sum_{j=1}^{n} w_{j, j}=1$, then there exists a unital homomorphism $\varphi: M_{n} \rightarrow B$ such that $\varphi\left(e_{j, j}\right)=w_{j, j}$ for $1 \leq j \leq n$ and $\left\|\varphi\left(e_{j, k}\right)-w_{j, k}\right\|<\varepsilon$ for $1 \leq j, k \leq n$.

Proof. This follows from semiprojectivity of $M_{n}$ (see Lemma 14.1.5, Theorem 14.2.2, Theorem 14.1.4, and Definition 14.1.1 of [18]), and the fact that if two families of $n$ orthogonal projections summing to 1 are close then there is a unitary close to 1 which conjugates one family to the other (Lemma 2.5.7 of [16]).

Theorem 2.2. Let $A$ be a unital AF algebra. Let $\alpha: G \rightarrow \operatorname{Aut}(A)$ be an action of a finite group $G$ on $A$ which has the strict Rokhlin property. Then $C^{*}(G, A, \alpha)$ is an AF algebra.

Proof. We prove that for every finite set $S \subset C^{*}(G, A, \alpha)$ and every $\varepsilon>0$, there is an AF subalgebra $D \subset C^{*}(G, A, \alpha)$ such that every element of $S$ is within $\varepsilon$ of an element of $D$. It is then easy to use Theorem 2.2 of 4 to show that $C^{*}(G, A, \alpha)$ is AF. It suffices to consider a finite set of the form $S=F \cup\left\{u_{g}: g \in G\right\}$, where $F$ 
is a finite subset of the unit ball of $A$ and $u_{g} \in C^{*}(G, A, \alpha)$ is the canonical unitary implementing the automorphism $\alpha_{g}$. So let $F \subset A$ be a finite subset with $\|a\| \leq 1$ for all $a \in F$ and let $\varepsilon>0$.

Set $n=\operatorname{card}(G)$, and set $\varepsilon_{0}=\varepsilon /(4 n)$. Choose $\delta>0$ according to Lemma 2.1 for $n$ as given and for $\varepsilon_{0}$ in place of $\varepsilon$. Also require $\delta \leq \varepsilon /[2 n(n+1)]$. Apply the strict Rokhlin property to $\alpha$ with $F$ as given and with $\delta$ in place of $\varepsilon$, obtaining projections $e_{g} \in A$ for $g \in G$. Define $w_{g, h}=u_{g^{-1}} e_{h}$ for $g, h \in G$.

We claim that the $w_{g, h}$ form a $\delta$-approximate system of $n \times n$ matrix units in $C^{*}(G, A, \alpha)$. We estimate:

$$
\left\|w_{g, h}^{*}-w_{h, g}\right\|=\left\|e_{h} u_{g h^{-1}}^{*}-u_{h g^{-1}} e_{g}\right\|=\left\|u_{g h^{-1}} e_{h} u_{g h^{-1}}^{*}-e_{g}\right\|=\left\|\alpha_{g h^{-1}}\left(e_{h}\right)-e_{g}\right\|<\delta .
$$

Also, using $e_{g} e_{h}=\delta_{g, h} e_{h}$ at the second step,

$$
\begin{aligned}
\left\|w_{g_{1}, h_{1}} w_{g_{2}, h_{2}}-\delta_{g_{2}, h_{1}} w_{g_{1}, h_{2}}\right\| & =\left\|u_{g_{1} h_{1}^{-1}} e_{h_{1}} u_{g_{2} h_{2}^{-1}} e_{h_{2}}-\delta_{g_{2}, h_{1}} u_{g_{1} h_{2}^{-1}} e_{h_{2}}\right\| \\
& =\left\|u_{g_{1} h_{1}^{-1}} e_{h_{1}} u_{g_{2} h_{2}^{-1}} e_{h_{2}}-u_{g_{1} h_{1}^{-1} g_{2} h_{2}^{-1}} e_{h_{2} g_{2}^{-1} h_{1}} e_{h_{2}}\right\| \\
& =\left\|u_{g_{1} h_{1}^{-1} g_{2} h_{2}^{-1}}\left(u_{g_{2} h_{2}^{-1}}^{*} e_{h_{1}} u_{g_{2} h_{2}^{-1}}-e_{h_{2} g_{2}^{-1} h_{1}}\right) e_{h_{2}}\right\|<\delta .
\end{aligned}
$$

Finally, $\sum_{g \in G} w_{g, g}=\sum_{g \in G} e_{g}=1$. This proves the claim.

Let $\left(v_{g, h}\right)_{g, h \in G}$ be a system of matrix units for $M_{n}$. By the choice of $\delta$, there exists a unital homomorphism $\varphi_{0}: M_{n} \rightarrow C^{*}(G, A, \alpha)$ such that $\left\|\varphi_{0}\left(v_{g, h}\right)-w_{g, h}\right\|<\varepsilon_{0}$ for all $g, h \in G$, and $\varphi_{0}\left(v_{g, g}\right)=e_{g}$ for all $g \in G$. Now define a unital homomorphism $\varphi: M_{n} \otimes e_{1} A e_{1} \rightarrow C^{*}(G, A, \alpha)$ by $\varphi\left(v_{g, h} \otimes a\right)=\varphi_{0}\left(v_{g, 1}\right) a \varphi_{0}\left(v_{1, h}\right)$ for $g, h \in G$ and $a \in e_{1} A e_{1}$. It is well known that a corner of an $\mathrm{AF}$ algebra is $\mathrm{AF}$, and $\varphi$ is injective, so $D=\varphi\left(M_{n} \otimes e_{1} A e_{1}\right)$ is an AF subalgebra of $C^{*}(G, A, \alpha)$. We complete the proof by showing that every element of $S$ is within $\varepsilon$ of an element of $D$.

For $g \in G$ we have $\sum_{h \in G} \varphi_{0}\left(v_{g h, h}\right) \in D$ and

$$
\begin{aligned}
\left\|u_{g}-\sum_{h \in G} \varphi_{0}\left(v_{g h, h}\right)\right\| & \leq \sum_{h \in G}\left\|u_{g} e_{h}-\varphi_{0}\left(v_{g h, h}\right)\right\| \\
& =\sum_{h \in G}\left\|w_{g h, h}-\varphi_{0}\left(v_{g h, h}\right)\right\|<n \varepsilon_{0} \leq \varepsilon .
\end{aligned}
$$

Now let $a \in F$. Set

$$
b=\sum_{g \in G} v_{g, g} \otimes e_{1} \alpha_{g}^{-1}(a) e_{1} \in M_{n} \otimes e_{1} A e_{1} .
$$

Using $\left\|e_{g} a e_{h}\right\| \leq\left\|\left[e_{g}, a\right]\right\|+\left\|a e_{g} e_{h}\right\|$, we get

$$
\left\|a-\sum_{g \in G} e_{g} a e_{g}\right\| \leq \sum_{g \neq h}\left\|e_{g} a e_{h}\right\|<n(n-1) \delta .
$$

We use this, and the inequalities

$$
\left\|\varphi_{0}\left(v_{g, 1}\right) e_{1}-u_{g} e_{1}\right\|<\varepsilon_{0} \quad \text { and } \quad\left\|e_{1} \alpha_{g}^{-1}(a) e_{1}-\alpha_{g}^{-1}\left(e_{g} a e_{g}\right)\right\|<2 \delta,
$$


to get

$$
\begin{aligned}
\|a-\varphi(b)\| & =\left\|a-\sum_{g \in G} \varphi_{0}\left(v_{g, 1}\right) e_{1} \alpha_{g}^{-1}(a) e_{1} \varphi_{0}\left(v_{1, g}\right)\right\| \\
& <2 n \varepsilon_{0}+\left\|a-\sum_{g \in G} u_{g} e_{1} \alpha_{g}^{-1}(a) e_{1} u_{g}^{*}\right\| \\
& <2 n \varepsilon_{0}+2 n \delta+\left\|a-\sum_{g \in G} u_{g} \alpha_{g}^{-1}\left(e_{g} a e_{g}\right) u_{g}^{*}\right\| \\
& <2 n \varepsilon_{0}+2 n \delta+n(n-1) \delta \leq \varepsilon .
\end{aligned}
$$

This completes the proof.

The following result gives the criterion we use for a simple separable unital $\mathrm{C}^{*}$ algebra to have tracial rank zero. Note that, by Theorem 7.1(a) of [15], tracial rank zero is the same as tracially AF in the sense of Definition 2.1 of [14].

Proposition 2.3. Let $A$ be a simple separable unital $\mathrm{C}^{*}$-algebra. Then $A$ has tracial rank zero in the sense of Definition 3.1 of [15] if and only if the following holds.

For every finite set $F \subset A$, every $\varepsilon>0$, and every nonzero positive element $x \in A$, there is a projection $p \in A$ and a finite dimensional unital subalgebra $E \subset p A p$ (that is, $p$ is the identity of $E$ ) such that:

(1) $\|p a-a p\|<\varepsilon$ for all $a \in F$.

(2) For every $a \in F$ there exists $b \in E$ such that $\|p a p-b\|<\varepsilon$.

(3) $1-p$ is Murray-von Neumann equivalent to a projection in $\overline{x A x}$.

Proof. Theorem 6.13 and Definition 3.4 of [15] give this result, except with unitary equivalence instead of Murray-von Neumann equivalence in (3). However, according to Remark 6.12 and Theorem 6.9 of [15], with (3) as it stands, the resulting condition implies that $A$ has stable rank one.

The condition of Proposition 2.3 is given as the definition in [16. See Definition 3.6.2 there.

For convenient reference, we recall some properties of simple unital $\mathrm{C}^{*}$-algebras with tracial rank zero. (Most of them will not be needed until later.)

Definition 2.4. Let $A$ be a unital $\mathrm{C}^{*}$-algebra. We say that the order on projections over $A$ is determined by traces if whenever $n \in \mathbb{N}$ and $p, q \in M_{n}(A)$ are projections such that $\tau(p)<\tau(q)$ for all tracial states $\tau$ on $A$, then $p \precsim q$.

This is just Blackadar's Second Fundamental Comparability Question for all matrix algebras over $A$. See 1.3.1 in 3 .

Theorem 2.5. (H. Lin.) Let $A$ be a simple separable unital $\mathrm{C}^{*}$-algebra with tracial rank zero. Then $A$ has real rank zero and stable rank one. Moreover, the order on projections over $A$ is determined by traces (Definition [2.4).

Proof. In view of Theorem 7.1(a) of [15], real rank zero and stable rank one are Theorem 3.4 of [14]. That the order is determined by traces is Corollary 5.7 and Theorems 5.8 and 6.8 of [15].

Theorem 2.6. Let $A$ be an infinite dimensional simple separable unital $\mathrm{C}^{*}$-algebra with tracial rank zero. Let $\alpha: G \rightarrow \operatorname{Aut}(A)$ be an action of a finite group $G$ on $A$ which has the tracial Rokhlin property. Then $C^{*}(G, A, \alpha)$ has tracial rank zero. 
Proof. The proof is a modification of that of Theorem 2.2. It suffices to verify the condition of Proposition 2.3 for a finite set $S$ of the form $S=F \cup\left\{u_{g}: g \in G\right\}$, where $F$ is a finite subset of the unit ball of $A$ and $u_{g} \in C^{*}(G, A, \alpha)$ is the canonical unitary implementing the automorphism $\alpha_{g}$. So let $F \subset A$ be a finite subset with $\|a\| \leq 1$ for all $a \in F$, let $\varepsilon>0$, and let $x \in C^{*}(G, A, \alpha)$ be a nonzero positive element.

The $\mathrm{C}^{*}$-algebra $A$ has Property (SP) by Theorem 2.5 So Proposition 1.12 and Corollary [1.6 provide a nonzero projection $q \in A$ which is Murray-von Neumann equivalent in $C^{*}(G, A, \alpha)$ to a projection in $\overline{x C^{*}(G, A, \alpha) x}$. By Lemma 1.10 there are orthogonal nonzero projections $q_{1}, q_{2} \in A$ such that $q_{1}, q_{2} \leq q$.

Set $n=\operatorname{card}(G)$, and set $\varepsilon_{0}=\varepsilon /(16 n)$. Choose $\delta>0$ according to Lemma 2.1 for $n$ as given and for $\varepsilon_{0}$ in place of $\varepsilon$. Also require $\delta \leq \varepsilon /[8 n(n+1)]$. Apply Lemma 1.17 to $\alpha$, with $F$ as given, with $\delta$ in place of $\varepsilon$, and with $q_{1}$ in place of $x$, obtaining projections $e_{g} \in A$ for $g \in G$. Set $e=\sum_{g \in G} e_{g}$. By construction, $u_{g} e u_{g}^{*}=\alpha_{g}(e)=e$ for every $g \in G$. Also, for $a \in F$ we have $\|e a-a e\| \leq \sum_{g \in G}\left\|e_{g} a-a e_{g}\right\|<n \varepsilon_{0}$.

Define $w_{g, h}=u_{g h^{-1}} e_{h}$ for $g, h \in G$. Using the same estimates as in the proof of Theorem 2.2 we find an injective unital homomorphism $\varphi: M_{n} \otimes e_{1} A e_{1} \rightarrow$ $e C^{*}(G, A, \alpha) e$ and a finite set $T$ in the closed unit ball of $M_{n} \otimes e_{1} A e_{1}$ such that for every $a \in S=F \cup\left\{u_{g}: g \in G\right\}$, there is $b \in T$ such that $\|\varphi(b)-e a e\|<\frac{1}{4} \varepsilon$. Let $e_{1,1} \in M_{n}$ denote the usual $(1,1)$ matrix unit. Then $\varphi$ furthermore has the property that if $a \in e_{1} A e_{1}$ then $\varphi\left(e_{1,1} \otimes a\right)=a$. Use Lemma 1.9 to choose equivalent nonzero projections $f_{1}, f_{2} \in A$ such that $f_{1} \leq e_{1}$ and $f_{2} \leq q_{2}$. It follows from Theorems 3.10 and 3.12(1) of 14] that $M_{n} \otimes e_{1} A e_{1}$ has tracial rank zero, so there is a projection $p_{0} \in M_{n} \otimes e_{1} A e_{1}$ and a finite dimensional unital subalgebra $E_{0} \subset p_{0}\left(M_{n} \otimes e_{1} A e_{1}\right) p_{0}$ such that $\left\|p_{0} b-b p_{0}\right\|<\frac{1}{4} \varepsilon$ for all $b \in T$, such that for every $b \in T$ there exists $c \in E_{0}$ with $\left\|p_{0} b p_{0}-c\right\|<\frac{1}{4} \varepsilon$, and such that $1-p_{0} \precsim e_{1,1} \otimes f_{1}$ in $M_{n} \otimes e_{1} A e_{1}$. Set $p=\varphi\left(p_{0}\right)$, and set $E=\varphi\left(E_{0}\right)$, which is a finite dimensional unital subalgebra of $p C^{*}(G, A, \alpha) p$.

Let $a \in S$. Choose $b \in T$ such that $\|\varphi(b)-e a e\|<\frac{1}{4} \varepsilon$. Then, using $p e=e p=p$,

$$
\begin{aligned}
\|p a-a p\| & \leq 2\|e a-a e\|+\|p e a e-e a e p\| \\
& \leq 2\|e a-a e\|+2\|e a e-\varphi(b)\|+\left\|p_{0} b-b p_{0}\right\|<2 n \varepsilon_{0}+2\left(\frac{1}{4} \varepsilon\right)+\frac{1}{4} \varepsilon \leq \varepsilon .
\end{aligned}
$$

Further, choosing $c \in E_{0}$ such $\left\|p_{0} b p_{0}-c\right\|<\frac{1}{4} \varepsilon$, the element $\varphi(c)$ is in $E$ and satisfies

$$
\|p a p-\varphi(c)\| \leq\|e a e-\varphi(b)\|+\left\|p_{0} b p_{0}-c\right\|<\frac{1}{4} \varepsilon+\frac{1}{4} \varepsilon \leq \varepsilon .
$$

Finally, in $C^{*}(G, A, \alpha)$ we have

$$
1-p=(1-e)+(e-p) \precsim q_{1}+f_{2} \leq q,
$$

and $q$ is Murray-von Neumann equivalent to a projection in $\overline{x C^{*}(G, A, \alpha) x}$.

\section{TRACIALly APPROXIMATELY REPRESENTABLE ACTIONS AND DUALITY}

In this section, we give the tracial analog of approximate representability of an action, Definition 3.6(2) of [10. As there, we restrict to abelian groups; see Remark 3.7 of [10]. After several elementary properties and reformulations, we generalize Lemma 3.8 of [10], showing that an action is tracially approximately representable if and only if the dual action has the tracial Rokhlin property, and similarly with the action and its dual exchanged. 
Definition 3.6(2) of [10] is formulated in terms of central sequences, so we give a reformulation with direct estimates.

Lemma 3.1. Let $A$ be a separable unital $\mathrm{C}^{*}$-algebra, and let $\alpha: G \rightarrow \operatorname{Aut}(A)$ be an action of a finite abelian group $G$ on $A$. Then $\alpha$ is approximately representable (Definition 3.6(2) of [10]) if and only if for every finite set $F \subset A$ and every $\varepsilon>0$, there are unitaries $w_{g} \in A$ such that:

(1) $\left\|\alpha_{g}(a)-w_{g} a w_{g}^{*}\right\|<\varepsilon$ for all $a \in F$ and all $g \in G$.

(2) $\left\|w_{g} w_{h}-w_{g h}\right\|<\varepsilon$ for all $g, h \in G$.

(3) $\left\|\alpha_{g}\left(w_{h}\right)-w_{h}\right\|<\varepsilon$ for all $g, h \in G$.

Proof. It is immediate that the condition of the lemma implies approximate representability.

Now assume $\alpha$ is approximately representable, and let $F \subset A$ be finite and let $\varepsilon>0$. Without loss of generality $\|a\| \leq 1$ for all $a \in F$. Set $n=\operatorname{card}(G)$. Using semiprojectivity of $C^{*}(G) \cong \mathbb{C}^{n}$ (see Lemma 14.1.5, Theorem 14.2.1, Theorem 14.1.4, and Definition 14.1.1 of [18]), choose $\delta>0$ such that whenever $A$ is a unital C*-algebra and elements $x_{g} \in A$, for $g \in G$, satisfy $\left\|x_{g} x_{h}-x_{g h}\right\|<\delta$ for all $g, h \in G$ and $\left\|x_{g}^{*} x_{g}-1\right\|,\left\|x_{g} x_{g}^{*}-1\right\|<\delta$ for all $g \in G$, then there exist unitaries $w_{g} \in A$ such that $\left\|w_{g} w_{h}-w_{g h}\right\|<\varepsilon$ for all $g, h \in G$ and $\left\|w_{g}-x_{g}\right\|<\frac{1}{3} \varepsilon$ for all $g \in G$. From Definition 3.6(2) of [10], we get elements $x_{g} \in A$ such that the conditions above are satisfied, and also $\left\|\alpha_{g}(a)-x_{g} a x_{g}^{*}\right\|<\frac{1}{3} \varepsilon$ for all $a \in F$ and all $g \in G$ and $\left\|\alpha_{g}\left(x_{h}\right)-x_{h}\right\|<\frac{1}{3} \varepsilon$ for all $g, h \in G$. Moreover, we may clearly require $\left\|x_{g}\right\| \leq 1$ for all $g \in G$. It is now easy to check that Conditions (1) through (3) hold.

We now give the tracial analog.

Definition 3.2. Let $A$ be an infinite dimensional simple separable unital $\mathrm{C}^{*}$ algebra, and let $\alpha: G \rightarrow \operatorname{Aut}(A)$ be an action of a finite abelian group $G$ on $A$. We say that $\alpha$ is tracially approximately representable if for every finite set $F \subset A$, every $\varepsilon>0$, and every positive element $x \in A$ with $\|x\|=1$, there are a projection $e \in A$ and unitaries $w_{g} \in e A e$ such that:

(1) $\|e a-a e\|<\varepsilon$ for all $a \in F$.

(2) $\left\|\alpha_{g}(e a e)-w_{g} e a e w_{g}^{*}\right\|<\varepsilon$ for all $a \in F$ and all $g \in G$.

(3) $\left\|w_{g} w_{h}-w_{g h}\right\|<\varepsilon$ for all $g, h \in G$.

(4) $\left\|\alpha_{g}\left(w_{h}\right)-w_{h}\right\|<\varepsilon$ for all $g, h \in G$.

(5) $1-e$ is Murray-von Neumann equivalent to a projection in the hereditary subalgebra of $A$ generated by $x$.

(6) $\|$ exe $\|>1-\varepsilon$.

We next give several elementary properties and reformulations. In the rest of this section, we generalize Lemma 3.8 of [10, showing that an action is tracially approximately representable if and only if the dual action has the tracial Rokhlin property, and similarly with the action and its dual exchanged.

Lemma 3.3. Let $A$ be an infinite dimensional simple separable unital $\mathrm{C}^{*}$-algebra, and let $\alpha: G \rightarrow \operatorname{Aut}(A)$ be an action of a finite abelian group $G$ on $A$. If $\alpha$ is approximately representable in the sense of Definition 3.6(2) of [10], than $\alpha$ is tracially approximately representable. If $\alpha$ is tracially approximately representable, then $A$ has Property (SP) or $\alpha$ is approximately representable. 
Proof. This is immediate from Lemma 3.1

When $A$ is finite, we do not need Condition (6) of Definition [3.2

Lemma 3.4. Let $A$ be an infinite dimensional finite simple separable unital $\mathrm{C}^{*}$ algebra, and let $\alpha: G \rightarrow \operatorname{Aut}(A)$ be an action of a finite abelian group $G$ on $A$. Then $\alpha$ is tracially approximately representable if and only if for every finite set $F \subset A$, every $\varepsilon>0$, and every positive element $x \in A$ with $\|x\|=1$, there are a projection $e \in A$ and unitaries $w_{g} \in e A e$ such that:

(1) $\|e a-a e\|<\varepsilon$ for all $a \in F$.

(2) $\left\|\alpha_{g}(e a e)-w_{g} e a e w_{g}^{*}\right\|<\varepsilon$ for all $a \in F$ and all $g \in G$.

(3) $\left\|w_{g} w_{h}-w_{g h}\right\|<\varepsilon$ for all $g, h \in G$.

(4) $\left\|\alpha_{g}\left(w_{h}\right)-w_{h}\right\|<\varepsilon$ for all $g, h \in G$.

(5) $1-e$ is Murray-von Neumann equivalent to a projection in the hereditary subalgebra of $A$ generated by $x$.

Proof. The proof is the same as for Lemma 1.16.

In the definition of tracial approximate representability, we can require invariance instead of approximate invariance, and we can require that $g \mapsto w_{g}$ be a homomorphism.

Lemma 3.5. Let $A$ be an infinite dimensional simple separable unital $\mathrm{C}^{*}$-algebra, and let $\alpha: G \rightarrow \operatorname{Aut}(A)$ be a tracially approximately representable action of a finite abelian group $G$ on $A$. Then for every finite set $F \subset A$, every $\varepsilon>0$, and every positive element $x \in A$ with $\|x\|=1$, there are an $\alpha$-invariant projection $e \in A$ and a homomorphism $g \mapsto w_{g}$ from $G$ to the unitary group of $e A e$ such that:

(1) $\|e a-a e\|<\varepsilon$ for all $a \in F$.

(2) $\left\|\alpha_{g}(e a e)-w_{g} e a e w_{g}^{*}\right\|<\varepsilon$ for all $a \in F$ and all $g \in G$.

(3) $w_{g} w_{h}=w_{g h}$ for all $g, h \in G$.

(4) $\alpha_{g}\left(w_{h}\right)=w_{h}$ for all $g, h \in G$.

(5) $1-e$ is Murray-von Neumann equivalent to a projection in the hereditary subalgebra of $A$ generated by $x$.

(6) $\|$ exe $\|>1-\varepsilon$.

Proof. Let $F, \varepsilon$, and $x$ be given. Without loss of generality $\|a\| \leq 1$ for all $a \in F$.

The group algebra $C^{*}(G)$ is finite dimensional, hence semiprojective. (See Lemma 14.1.5 and Theorems 14.2.1 and 14.2.2 of [18.) In particular, by Theorem 14.1.4 of [18], its standard generators $u_{g}$ for $g \in G$, and relations $\left(u_{g}\right.$ is unitary and $u_{g} u_{h}=u_{g h}$ for $\left.g, h \in G\right)$ are stable in the sense of Definition 14.1.1 of [18]. Accordingly, there exists $\varepsilon_{0}>0$ such that whenever $\left(x_{g}\right)_{g \in G}$ is a collection of elements of a unital $\mathrm{C}^{*}$-algebra $B$ such that for all $g, h \in G$ we have

$$
\left\|x_{g} x_{g}^{*}-1\right\|<10 \varepsilon_{0}, \quad\left\|x_{g}^{*} x_{g}-1\right\|<10 \varepsilon_{0}, \quad \text { and } \quad\left\|x_{g} x_{h}-x_{g h}\right\|<10 \varepsilon_{0},
$$

then there exists a unital homomorphism $\varphi: C^{*}(G) \rightarrow B$ such that $\left\|\varphi\left(u_{g}\right)-x_{g}\right\|<$ $\frac{1}{13} \varepsilon$ for all $g \in G$. We also require $\varepsilon_{0} \leq \min \left(\frac{1}{13} \varepsilon, 1\right)$.

Next, choose $\delta>0$ with $\delta \leq \varepsilon_{0}$ and so small that whenever $p$ is a projection in a $C^{*}$-algebra $B$, and $a \in B$ is selfadjoint and satisfies $\|a-p\|<3 \delta$, then the projection $q=\chi_{[1 / 2, \infty)}(a)$ is defined and satisfies $\|q-p\|<\varepsilon_{0}$.

Apply Definition 3.2 with $F$ as given, with $\delta$ in place of $\varepsilon$, and with $x$ as given, obtaining a projection $f$ and unitaries $y_{g} \in f A f$. Note that $\left\|y_{1}^{2}-y_{1}\right\|<\delta$, so 
$\left\|y_{1}-f\right\|<\delta$. Therefore $\left\|\alpha_{g}(f)-f\right\|<3 \delta$ for all $g \in G$. Consequently, the element

$$
b=\frac{1}{\operatorname{card}(G)} \sum_{h \in G} \alpha_{h}(f) \in A^{\alpha}
$$

satisfies $\|b-f\|<3 \delta$, so that there is a projection $e \in A^{\alpha}$ such that $\|e-f\|<\varepsilon_{0}$.

For every $g, h \in G$, we now have

$$
\left\|\alpha_{h}\left(e y_{g} e\right)-y_{g}\right\| \leq\left\|e y_{g} e-y_{g}\right\|+\left\|\alpha_{h}\left(y_{g}\right)-y_{g}\right\|<2\|e-f\|+\delta<3 \varepsilon_{0} .
$$

Therefore the elements

$$
x_{g}=\frac{1}{\operatorname{card}(G)} \sum_{h \in G} \alpha_{h}\left(e y_{g} e\right) \in(e A e)^{\alpha}
$$

satisfy $\left\|x_{g}-y_{g}\right\|<3 \varepsilon_{0}$. We now estimate:

$$
\left\|x_{g} x_{g}^{*}-f\right\| \leq 2\left\|x_{g}-y_{g}\right\|+\left\|y_{g} y_{g}^{*}-f\right\|<6 \varepsilon_{0}+0=6 \varepsilon_{0} ;
$$

similarly, $\left\|x_{g}^{*} x_{g}-1\right\|<6 \varepsilon_{0}$; and also

$\left\|x_{g} x_{h}-x_{g h}\right\| \leq\left\|x_{g}-y_{g}\right\|+\left\|x_{h}-y_{h}\right\|+\left\|x_{g h}-y_{g h}\right\|+\left\|y_{g} y_{h}-y_{g h}\right\|<9 \varepsilon_{0}+\delta \leq 10 \varepsilon_{0}$.

So there is a unital homomorphism $\varphi: C^{*}(G) \rightarrow(e A e)^{\alpha}$ such that $\left\|\varphi\left(u_{g}\right)-x_{g}\right\|<$ $\frac{1}{13} \varepsilon$ for all $g \in G$. Set $w_{g}=\varphi\left(u_{g}\right)$. Then $\left\|w_{g}-y_{g}\right\|<\frac{1}{13} \varepsilon+3 \varepsilon_{0} \leq \frac{4}{13} \varepsilon$.

We now have, for $a \in F$,

$$
\|e a-a e\| \leq 2\|e-f\|+\|f a-a f\|<2 \varepsilon_{0}+\delta<\varepsilon,
$$

and, for $a \in F$ and $g \in G$,

$$
\begin{aligned}
\left\|\alpha_{g}(e a e)-w_{g} e a e w_{g}^{*}\right\| & \leq 4\|e-f\|+2\left\|w_{g}-y_{g}\right\|+\| \alpha_{g}(\text { faf })-y_{g} f a f y_{g}^{*} \| \\
& <4 \varepsilon_{0}+\frac{8}{13} \varepsilon+\delta \leq \varepsilon .
\end{aligned}
$$

This gives Parts (1)-(4) of the conclusion.

For Part (5),

$$
\|e x e\| \geq\|f x f\|-2\|e-f\|>1-\delta-2 \varepsilon_{0}>1-\varepsilon .
$$

For Part ([6]), use $\|e-f\|<\varepsilon_{0} \leq 1$ to get $e \sim f$.

We now turn to the proof of the duality relations. We will use Takai duality to get one part from the other, and to do so we need to know that an action of a finite abelian group has the tracial Rokhlin property if and only if the second dual action has the tracial Rokhlin property. The next few lemmas contain the proof of this fact.

We must identify what happens to the inclusion map $A \rightarrow C^{*}\left(\widehat{G}, C^{*}(G, A, \alpha), \widehat{\alpha}\right)$ under Takai duality [27]. The formula in the next proposition is correct without simplicity, but assuming simplicity shortens the proof.

Proposition 3.6. Let $A$ be a simple unital $\mathrm{C}^{*}$-algebra, and let $\alpha: G \rightarrow \operatorname{Aut}(A)$ be an action of a finite abelian group $G$ on $A$. In $L\left(l^{2}(G)\right)$, let $\left(e_{g, h}\right)_{g, h \in G}$ be the family of matrix units determined by the requirement that $e_{g, h}$ send the standard basis vector $\delta_{h}$ to the standard basis vector $\delta_{g}$, and vanish on all other standard basis vectors. Let

$$
\iota: A \rightarrow C^{*}(G, A, \alpha) \text { and } \quad \mu: C^{*}(G, A, \alpha) \rightarrow C^{*}\left(\widehat{G}, C^{*}(G, A, \alpha), \widehat{\alpha}\right)
$$


be the inclusions. Then there exists an isomorphism $\varphi: C^{*}\left(\widehat{G}, C^{*}(G, A, \alpha), \widehat{\alpha}\right) \rightarrow$ $L\left(l^{2}(G)\right) \otimes A$ such that

$$
(\varphi \circ \mu \circ \iota)(a)=\sum_{k \in G} e_{k, k} \otimes \alpha_{k}^{-1}(a)
$$

for all $a \in A$.

Proof. For $g \in G$ let $u_{g} \in C^{*}(G, A, \alpha)$ be the standard implementing unitary, and for $\tau \in \widehat{G}$ let $v_{\tau} \in C^{*}\left(\widehat{G}, C^{*}(G, A, \alpha), \widehat{\alpha}\right)$ be the standard implementing unitary. Identify $A$ and $C^{*}(G, A, \alpha)$ with their images in $C^{*}\left(\widehat{G}, C^{*}(G, A, \alpha), \widehat{\alpha}\right)$ under $\mu \circ \iota$ and $\mu$. Then define $\varphi$ on the generators of $C^{*}\left(\widehat{G}, C^{*}(G, A, \alpha), \widehat{\alpha}\right)$ by

$\varphi(a)=\sum_{k \in G} e_{k, k} \otimes \alpha_{k}^{-1}(a), \quad \varphi\left(u_{g}\right)=\sum_{k \in G} e_{g k, k} \otimes 1, \quad$ and $\quad \varphi\left(v_{\tau}\right)=\sum_{k \in G} e_{k, k} \otimes \tau(k)$

for $a \in A, g \in G$, and $\tau \in \widehat{G}$. One checks that the appropriate relations are satisfied for this definition to extend to a homomorphism, and it is easily seen that the resulting homomorphism is surjective. It is injective because, by Takai duality [27, simplicity of $A$ implies simplicity of $C^{*}\left(\widehat{G}, C^{*}(G, A, \alpha), \widehat{\alpha}\right)$.

Lemma 3.7. Let $A$ be an infinite dimensional simple separable unital $\mathrm{C}^{*}$-algebra, and let $\alpha: G \rightarrow \operatorname{Aut}(A)$ be an action of a finite group $G$ on $A$ which has the tracial Rokhlin property. Let $p \in A$ be a $G$-invariant projection. Then the action $\left.g \mapsto \alpha_{g}\right|_{p A p}$ has the tracial Rokhlin property.

Proof. Let $F \subset p A p$ be finite, let $\varepsilon>0$, and let $x \in p A p$ be a positive element with $\|x\|=1$. Set $n=\operatorname{card}(G)$. Set

$$
\varepsilon_{0}=\min \left(\frac{1}{n}, \frac{\varepsilon}{4 n+1}\right) .
$$

Using semiprojectivity of $\mathbb{C}^{n}$, choose $\delta>0$ such that whenever $B$ is a unital $\mathrm{C}^{*}$ algebra, $q_{1}, \ldots, q_{n} \in B$ are mutually orthogonal projections, and $p \in B$ is a projection such that $\left\|p q_{j}-q_{j} p\right\|<\delta$ for $1 \leq j \leq n$, then there are mutually orthogonal projections $e_{j} \in p B p$ such that $\left\|e_{j}-p q_{j} p\right\|<\varepsilon_{0}$ for $1 \leq j \leq n$. We also require $\delta \leq \varepsilon_{0}$.

Apply Definition 1.2 to $\alpha$, with $F \cup\{p\}$ in place of $F$, with $\delta$ in place of $\varepsilon$, and with $x$ as given, obtaining projections $q_{g} \in A$ for $g \in G$. By the choice of $\delta$, there are mutually orthogonal projections $e_{g} \in p A p$ such that $\left\|e_{g}-p q_{g} p\right\|<\varepsilon_{0}$ for $g \in G$. We now estimate, using $\alpha_{g}(p)=p$,

$\left\|\alpha_{g}\left(e_{h}\right)-e_{g h}\right\| \leq\left\|e_{h}-p q_{h} p\right\|+\left\|e_{g h}-p q_{g h} p\right\|+\left\|p\left(\alpha_{g}\left(q_{h}\right)-q_{g h}\right) p\right\|<2 \varepsilon_{0}+\delta \leq \varepsilon$, and for $a \in F$, using $p a=a p=a$,

$$
\left\|e_{g} a-a e_{g}\right\| \leq 2\left\|e_{g}-p q_{g} p\right\|+\left\|p\left(q_{g} a-a q_{g}\right) p\right\|<2 \varepsilon_{0}+\delta \leq \varepsilon .
$$

Next, set $e=\sum_{g \in G} e_{g}$ and $q=\sum_{g \in G} q_{g}$. Then $\|e-p q p\|<n \varepsilon_{0} \leq 1$. So

$$
\begin{aligned}
\|(1-q)(p-e)-(p-e)\| & =\|q(p-e)\|=\|(p-e) q(p-e)\|^{1 / 2} \\
& =\|(1-e) p q p(1-e)\|^{1 / 2} \leq\|e-p q p\|^{1 / 2}<1 .
\end{aligned}
$$

It follows from Lemma 2.5.2 of [16] that $p-e \precsim 1-q$. Since $1-q$ is Murray-von Neumann equivalent to a projection in $\overline{x p A p x}=\overline{x A x}$, so is $p-e$. 
Finally, we estimate $\|e x e\|$. From $\left\|q_{g} p-p q_{g}\right\|<\delta \leq \varepsilon_{0}$ for all $g$, we get $\|q p-p q\|<$ $n \varepsilon_{0}$, whence $\|e-p q\|,\|e-q p\|<2 n \varepsilon_{0}$. Therefore

$$
\| \text { exe }\|>\| q p x p q\left\|-4 n \varepsilon_{0}=\right\| q x q \|-4 n \varepsilon_{0}>1-\delta-4 n \varepsilon_{0} \geq 1-\varepsilon .
$$

This completes the proof.

Lemma 3.8. Let $A$ be a $\mathrm{C}^{*}$-algebra, let $n \in \mathbb{N}$, and let $x=\left(x_{j, k}\right)_{1 \leq j, k \leq n} \in M_{n}(A)$ be positive. Then there exists $k$ such that $\left\|x_{k, k}\right\| \geq n^{-2}\|x\|$.

Proof. We first claim that if

$$
y=\left(\begin{array}{cc}
a & b \\
b^{*} & c
\end{array}\right) \in M_{2}(A)
$$

is positive, then $\|b\| \leq \frac{1}{2}(\|a\|+\|c\|)$. Without loss of generality $A \subset L(H)$ for some Hilbert space $H$, and we correspondingly take $M_{2}(A) \subset L\left(H^{2}\right)$. Let $\xi, \eta \in H$ satisfy $\|\xi\|=\|\eta\|=1$. Choose $t \in \mathbb{C}$ with $|t|=1$ such that $t\langle b \eta, \xi\rangle=-|\langle b \eta, \xi\rangle|$. Then one calculates that

$$
0 \leq\langle y(\xi, t \eta),(\xi, t \eta)\rangle=\langle a \xi, \xi\rangle+\langle c \eta, \eta\rangle-2|\langle b \eta, \xi\rangle| \leq\|a\|+\|c\|-2|\langle b \xi, \eta\rangle| .
$$

Therefore $|\langle b \eta, \xi\rangle| \leq \frac{1}{2}(\|a\|+\|c\|)$. The claim follows by taking the supremum over all $\xi, \eta \in H$ with $\|\xi\|=\|\eta\|=1$.

By considering $2 \times 2$ submatrices of $x$, the claim implies that $\left\|x_{j, k}\right\| \leq \frac{1}{2}\left(\left\|x_{j, j}\right\|+\right.$ $\left.\left\|x_{k, k}\right\|\right)$ for $1 \leq j, k \leq n$. Sum over all $j$ and $k$ to get

$$
\|x\| \leq \sum_{1 \leq j, k \leq n}\left\|x_{j, k}\right\| \leq n \sum_{k=1}^{n}\left\|x_{k, k}\right\| .
$$

The statement of the lemma follows.

Lemma 3.9. Let $A$ be an infinite dimensional simple separable unital $\mathrm{C}^{*}$-algebra, let $\alpha: G \rightarrow \operatorname{Aut}(A)$ be an action of a finite group $G$ on $A$, and let $g \mapsto v_{g}$ be a unitary representation of $G$ on $\mathbb{C}^{n}$. Then $\alpha$ has the tracial Rokhlin property if and only if the action $g \mapsto \operatorname{Ad}\left(v_{g}\right) \otimes \alpha_{g}$ of $G$ on $M_{n} \otimes A$ has the tracial Rokhlin property.

Proof. We first prove that if $\alpha$ has the tracial Rokhlin property, then so does $g \mapsto$ $\operatorname{Ad}\left(v_{g}\right) \otimes \alpha_{g}$. If $M_{n} \otimes A$ does not have Property (SP), then, by Lemma 1.11 neither does $A$, so $\alpha$ has the strict Rokhlin property by Lemma 1.13 Then we must show that $g \mapsto \operatorname{Ad}\left(v_{g}\right) \otimes \alpha_{g}$ has the strict Rokhlin property. The proof is similar to but easier than the other case, and is omitted.

So assume that $M_{n} \otimes A$ has Property (SP) and that $\alpha$ has the tracial Rokhlin property. Let $F \subset M_{n} \otimes A$ be finite, let $\varepsilon>0$, and let $x \in M_{n} \otimes A$ be a positive element with $\|x\|=1$. Let $\left(e_{j, k}\right)_{1 \leq j, k \leq n}$ be the standard system of matrix units for $M_{n}$. Without loss of generality we may assume that there is a finite set $F_{0} \subset A$ such that $\|a\| \leq 1$ for all $a \in F_{0}$ and such that

$$
F=\left\{e_{j, k} \otimes a: 1 \leq j, k \leq n \text { and } a \in F_{0}\right\} .
$$

Set $\varepsilon_{0}=\min \left(\varepsilon / 4,1 /\left(4 n^{2}\right)\right)$. Choose $\varepsilon_{1}>0$ with $\varepsilon_{1} \leq \varepsilon_{0}$ and so small that if $B$ is a $\mathrm{C}^{*}$-algebra and $e, q \in B$ are projections such that $\|e q-q e\|<\varepsilon_{1}$, then there is a projection $p \in B$ such that $\|e q e-p\|<\varepsilon_{0}$. Apply Lemma 1.14 obtaining a nonzero projection $p \in \overline{x\left(M_{n} \otimes A\right) x}$ such that $\left\|p_{0} x p_{0}-p_{0}\right\|<\varepsilon_{0}$ for every nonzero projection $p_{0} \leq p$. 
Write $p=\sum_{j, k=1}^{n} e_{j, k} \otimes p_{j, k}$. By Lemma 3.8, there is $k$ such that $\lambda=\left\|p_{k, k}\right\| \geq$ $n^{-2}$. Without loss of generality $k=1$. Lemma 1.11 provides a nonzero projection $d \in A$ such that $1 \otimes d \precsim p$. Then $q=e_{1,1} \otimes d$ is a nonzero projection in $\mathbb{C}_{1,1} \otimes$ $A$ such that $q \precsim p$. By Lemma 1.10 there exist nonzero Murray-von Neumann equivalent mutually orthogonal projections $q_{1}, q_{2}, \ldots, q_{n} \leq q$. Set $y=\lambda^{-1} p_{1,1}$. Apply Lemma 1.14 obtaining a nonzero projection $z \in \overline{y A y}$ such that $\left\|q_{0} y q_{0}-q_{0}\right\|<$ $\frac{1}{8}$ for every nonzero projection $q_{0} \leq z$. Using Property (SP) and Lemma 1.9] choose a nonzero projection $q_{0} \leq z$ such that $e_{1,1} \otimes q_{0} \precsim q_{1}$.

Set

$$
\delta=\min \left(\varepsilon, \frac{\varepsilon_{1}}{n^{2} \operatorname{card}(G)}, \frac{1}{4 n}, \frac{1}{8 \operatorname{card}(G)}\right) .
$$

Apply Definition 1.2 to $\alpha$, with $F_{0} \cup\left\{q_{0}\right\} \cup\left\{p_{j, k}: 1 \leq j, k \leq n\right\}$ in place of $F$, with $\delta$ in place of $\varepsilon$, and with $q_{0}$ in place of $x$. Let $f_{g}$, for $g \in G$, be the resulting projections. As usual, set $f=\sum_{g \in G} f_{g}$. Then define $e_{g}=1 \otimes f_{g}$. Since $\delta \leq \varepsilon$, it is immediate that $\left\|\left(\operatorname{Ad}\left(v_{g}\right) \otimes \alpha_{g}\right)\left(e_{h}\right)-e_{g h}\right\|<\varepsilon$ for all $g, h \in G$, and that $\left\|\left[e_{g},\left(e_{j, k} \otimes a\right)\right]\right\|<\varepsilon$ for all $g \in G$ and all $a \in F_{0}$. Moreover, with $e=\sum_{g \in G} e_{g}$, we have

$$
1-e=\sum_{k=1}^{n} e_{k, k} \otimes(1-f) \precsim \sum_{k=1}^{n} e_{k, k} \otimes q_{0} \precsim \sum_{k=1}^{n} q_{k} \leq q,
$$

which is Murray-von Neumann equivalent to a projection in $\overline{x\left(M_{n} \otimes A\right) x}$.

It remains only to show that $\|$ exe $\|>1-\varepsilon$. We have $\left\|\left[f, p_{j, k}\right]\right\|<\varepsilon_{1} / n^{2}$ for $1 \leq j, k \leq n$, so $\|[e, p]\|<\varepsilon_{1}$. Therefore there is a projection $r \in M_{n} \otimes A$ such that $\|r-e p e\|<\varepsilon_{0}$. Now, assuming $r \neq 0$ at the last step,

$$
\| \text { exe }\|\geq\| \text { pexep }\|\geq\| \text { epxpe }\left\|-2 \varepsilon_{1} \geq\right\| \text { epe }\left\|-2 \varepsilon_{1}-\varepsilon_{0}>\right\| r \|-2 \varepsilon_{1}-2 \varepsilon_{0} \geq 1-\varepsilon \text {. }
$$

So we need only show that $r \neq 0$. It suffices to show that $\|$ epe $\|>\varepsilon_{0}$.

Using $\left\|f q_{0} f\right\|>1-\delta$ at the seventh step, we have

$$
\begin{aligned}
\| \text { epe } \| & \geq\left\|f p_{1,1} f\right\|=\lambda\|f y f\| \geq \frac{1}{n^{2}}\|f y f\| \geq \frac{1}{n^{2}}\left\|q_{0} f y f q_{0}\right\| \\
& \geq \frac{1}{n^{2}}\left(\left\|f q_{0} y q_{0} f\right\|-2 \operatorname{card}(G) \delta\right)>\frac{1}{n^{2}}\left(\left\|f q_{0} f\right\|-\frac{1}{8}-\frac{1}{8}\right) \\
& >\frac{1}{n^{2}}\left(1-\delta-\frac{1}{4}\right) \geq \frac{1}{4 n^{2}} \geq \varepsilon_{0} .
\end{aligned}
$$

This completes the proof that $g \mapsto \operatorname{Ad}\left(v_{g}\right) \otimes \alpha_{g}$ has the tracial Rokhlin property.

Now suppose that $g \mapsto \operatorname{Ad}\left(v_{g}\right) \otimes \alpha_{g}$ has the tracial Rokhlin property. Let $g \mapsto w_{g}$ be the contragredient representation. By what we already did, $g \mapsto \operatorname{Ad}\left(w_{g} \otimes v_{g}\right) \otimes$ $\alpha_{g}$, which is an action of $G$ on $M_{n^{2}} \otimes A$, has the tracial Rokhlin property. The one dimensional trivial representation of $G$ is a subrepresentation of $g \mapsto w_{g} \otimes v_{g}$, so there is an invariant projection $p \in M_{n^{2}}$ such that the restriction of $\operatorname{Ad}\left(w_{g} \otimes v_{g}\right) \otimes \alpha_{g}$ to $(p \otimes 1)\left(M_{n^{2}} \otimes A\right)(p \otimes 1) \cong A$ can be identified with $\alpha$. Now Lemma 3.7 implies that $\alpha$ has the tracial Rokhlin property.

Lemma 3.10. Let $A$ be a simple $\mathrm{C}^{*}$-algebra with Property (SP), and let $\alpha: G \rightarrow$ $\operatorname{Aut}(A)$ be an action of a finite group $G$ on $A$. For each $g \in G$, let $p_{g} \in A$ be a nonzero projection. Then there exists a nonzero projection $q \in A$ such that $q \precsim \alpha_{g}\left(p_{g}\right)$ for all $g \in G$.

Proof. Write $G=\left\{g_{j}: 0 \leq j \leq n-1\right\}$, with $g_{0}=1$. Using Property (SP) and Lemma 1.9 find a nonzero projection $e_{1} \leq p_{1}$ such that $e_{1} \precsim \alpha_{g_{1}}\left(p_{g_{1}}\right)$. In the same way, find a nonzero projection $e_{2} \leq e_{1}$ such that $e_{2} \precsim \alpha_{g_{2}}\left(p_{g_{2}}\right)$. Proceed 
inductively. Set $q=e_{n-1}$. Then $q$ is a nonzero projection such that $q \precsim \alpha_{g_{j}}\left(p_{g_{j}}\right)$ for $0 \leq j \leq n-1$, whence $q \precsim \alpha_{g}\left(p_{g}\right)$ for all $g \in G$.

The next result is the analog of Lemma 3.8 of [10].

Theorem 3.11. Let $A$ be an infinite dimensional simple separable unital $\mathrm{C}^{*}$ algebra, and let $\alpha: G \rightarrow \operatorname{Aut}(A)$ be an action of a finite abelian group $G$ on $A$ such that $C^{*}(G, A, \alpha)$ is also simple. Then:

(1) $\alpha$ has the tracial Rokhlin property if and only if $\widehat{\alpha}$ is tracially approximately representable.

(2) $\alpha$ is tracially approximately representable if and only if $\widehat{\alpha}$ has the tracial Rokhlin property.

Proof. Using Proposition 1.12 Lemma1.11 and Takai duality [27, we see that that either both $A$ and $C^{*}(G, A, \alpha)$ have Property (SP) or neither does. If neither does, by Remark 1.4 and Lemma [1.13] the statement is equivalent to the corresponding statement for the strict Rokhlin property and approximate representability, and follows from Lemma 3.8 of [10. Thus, we assume both have Property (SP).

We prove (2). Statement (11) will then follow from (2) for $\widehat{\alpha}$, by combining Takai duality 27] with Lemma 3.9

For $g \in G$ let $u_{g} \in C^{*}(G, A, \alpha)$ be the standard unitary of the crossed product. Also set $n=\operatorname{card}(G)$.

Suppose that $\alpha$ is tracially approximately representable. Let $F \subset C^{*}(G, A, \alpha)$ be finite, let $\varepsilon>0$, and let $x \in C^{*}(G, A, \alpha)$ be a positive element with $\|x\|=1$. Without loss of generality $F=F_{0} \cup\left\{u_{g}: g \in G\right\}$ for some finite subset $F_{0} \subset A$, such that $\|a\| \leq 1$ for all $a \in F_{0}$.

Use Proposition 1.12 and Lemma 1.14 to find a nonzero projection $q_{0}$ in the hereditary subalgebra $\overline{x C^{*}(G, A, \alpha) x}$ such that whenever $p \leq q_{0}$ is a nonzero projection, then $\|p x p\| \geq 1-\frac{1}{8} \varepsilon$. By Proposition 1.12 again, there is a nonzero projection $q \in A$ and a partial isometry $c \in C^{*}(G, A, \alpha)$ such that $c c^{*}=q$ and $c^{*} c \leq q_{0}$. Write $c=\sum_{g \in G} c_{g} u_{g}$ with $c_{g} \in A$. Note that $\left\|c_{g}\right\| \leq\|c\|=1$.

Set $\varepsilon_{0}=\varepsilon /(8 n)$. Apply Lemma 3.5 with $F_{0} \cup\left\{c_{g}: g \in G\right\}$ in place of $F$, with $\varepsilon_{0}$ in place of $\varepsilon$, and with $q$ in place of $x$. Let $e \in A$ and $w_{g} \in e A e$ be the resulting projection and unitaries. For $\sigma \in \widehat{G}$, define $e_{\sigma} \in C^{*}(G, A, \alpha)$ by

$$
e_{\sigma}=\frac{1}{n} \sum_{g \in G} \sigma(g) u_{g} w_{g}^{*}
$$

We show that the $e_{\sigma}$ are projections which verify the definition of the tracial Rokhlin property.

The unitaries $u_{g} \in C^{*}(G, A, \alpha)$ and $w_{h} \in e A e$ commute for all $g, h \in G$, because $\alpha_{g}\left(w_{h}\right)=w_{h}$. It is now easy to check that $e_{\sigma}^{*}=e_{\sigma}$. Next, let $\sigma, \tau \in \widehat{G}$. Then, changing the variable $g$ to $g h^{-1}$ at the second step,

$$
e_{\sigma} e_{\tau}=\frac{1}{n^{2}} \sum_{g, h \in G} \sigma(g) \tau(h) u_{g h} w_{g h}^{*}=\frac{1}{n^{2}} \sum_{g \in G}\left(\sum_{h \in G}\left(\sigma^{-1} \tau\right)(h)\right) \sigma(g) u_{g} w_{g}^{*} .
$$

We have $\sum_{h \in G}\left(\sigma^{-1} \tau\right)(h)=n$ if $\sigma=\tau$, and the sum is zero otherwise, so the $e_{\sigma}$ are mutually orthogonal projections. Moreover,

$$
\sum_{\sigma \in \widehat{G}} e_{\sigma}=u_{1} w_{1}^{*}=e .
$$


For $\tau \in \widehat{G}$, we have $\widehat{\alpha}_{\tau}\left(w_{g}\right)=w_{g}$ and $\widehat{\alpha}_{\tau}\left(u_{g}\right)=\tau(g) u_{g}$. Therefore $\widehat{\alpha}_{\tau}\left(e_{\sigma}\right)=e_{\tau \sigma}$ for $\sigma, \tau \in \widehat{G}$.

Next, we check the approximate commutation relations. For $g \in G$, because $G$ is abelian and $u_{g}$ commutes with each $w_{h}$, we have $e_{\tau} u_{g}=u_{g} e_{\tau}$ for all $\tau \in \widehat{G}$. For $a \in F_{0}$ and $\tau \in \widehat{G}$, we use the relation $u_{g} e a e u_{g}^{*}=\alpha_{g}(e a e)$ and the estimate $\left\|\alpha_{g}(e a e)-w_{g} e a e w_{g}^{*}\right\|<\varepsilon_{0}$ to get

$$
\begin{aligned}
\left\|e_{\tau} a-a e_{\tau}\right\| & \leq 2\|e a-a e\|+\left\|e_{\tau} e a e-e^{a e e_{\tau}}\right\| \\
& \leq 2\|e a-a e\|+\frac{1}{n} \sum_{g \in G}\left\|\sigma(g) u_{g} w_{g}^{*} e a e-\operatorname{eae} \sigma(g) u_{g} w_{g}^{*}\right\| \\
& <2 \varepsilon_{0}+\varepsilon_{0} \leq \varepsilon .
\end{aligned}
$$

Next, we observe that $1-e \precsim q$, so $1-e$ is Murray-von Neumann equivalent to a projection in $\overline{x C^{*}(G, A, \alpha) x}$.

Finally, we estimate $\|$ exe $\|$. First, since $e$ is $\alpha$-invariant, we have $u_{g} e=e u_{g}$ for all $g \in G$. Therefore

$$
\|c e-e c\| \leq \sum_{g \in G}\left\|c_{g} e-e c_{g}\right\|<\frac{1}{8} \varepsilon,
$$

whence $\left\|c^{*} c e-e c^{*} c\right\|<\frac{1}{4} \varepsilon$. Using the fact that $c^{*} c$ is a projection and $c^{*} c \leq q_{0}$ at the fourth step, we get

$$
\begin{aligned}
\|e x e\| & \geq\left\|c^{*} \operatorname{cexec}^{*} c\right\| \geq\left\|e c^{*} c x c^{*} c e\right\|-\frac{1}{2} \varepsilon>\left\|e c^{*} c e\right\|-\frac{5}{8} \varepsilon=\left\|(c e)(c e)^{*}\right\|-\frac{5}{8} \varepsilon \\
& >\left\|e c c^{*} e\right\|-\frac{7}{8} \varepsilon=\|e q e\|-\frac{7}{8} \varepsilon>1-\varepsilon_{0}-\frac{7}{8} \varepsilon \geq 1-\varepsilon
\end{aligned}
$$

This completes the proof that $\widehat{\alpha}$ has the tracial Rokhlin property.

Now assume that $\widehat{\alpha}$ has the tracial Rokhlin property. Let $F \subset A$ be a finite set, let $\varepsilon>0$, and let $x \in A$ be a positive element with $\|x\|=1$. Without loss of generality $\|a\| \leq 1$ for all $a \in F$. Apply Lemma 1.14 obtaining a nonzero projection $q \in \overline{x A x}$ such that $\|r x r-r\|<\frac{1}{2} \varepsilon$ for every nonzero projection $r \leq q$. Use Lemma 1.10] to find a family $\left(q_{g}\right)_{g \in G}$ of nonzero mutually orthogonal projections in $\overline{q A q}$. By Lemma [3.10] there exists a nonzero projection $p \in A$ such that $p \precsim \alpha_{g}\left(q_{g}\right)$ for all $g \in G$. Therefore there are nonzero projections $p_{g} \in A$ with $p_{g} \leq q_{g}$ such that $p_{g} \sim \alpha_{g}\left(p_{1}\right)$ for all $g \in G$.

Choose $\varepsilon_{0}>0$ so small that if $B$ is a unital $\mathrm{C}^{*}$-algebra and $y \in B$ satisfies

$$
\left\|y y^{*}-1\right\|<5 \varepsilon_{0} n \text { and }\left\|y^{*} y-1\right\|<5 \varepsilon_{0} n,
$$

then there is a unitary $w \in B$ such that $\|w-y\|<\frac{1}{7} \varepsilon$. We also require that $9 n^{2} \varepsilon_{0} \leq \frac{1}{7} \varepsilon$.

Apply Lemma 1.17 to $\widehat{\alpha}$ with $F \cup\left\{p_{1}\right\} \cup\left\{u_{g}: g \in G\right\}$ in place of $F$, with $\varepsilon_{0}$ in place of $\varepsilon$, and with $p_{1}$ in place of $x$, obtaining projections $e_{\tau}$ for $\tau \in \widehat{G}$. Let $e=\sum_{\tau \in \widehat{G}} e_{\tau}$. Note that $e \in A$ because $e$ is $\widehat{\alpha}$-invariant.

Define

$$
x_{g}=\sum_{\sigma \in \widehat{G}} \sigma(g) e_{\sigma} u_{g} e_{\sigma}
$$

for $g \in G$. Note that $x_{g} \in e C^{*}(G, A, \alpha) e$. We estimate as follows. For $g \in G$ and $\tau \in \widehat{G}$,

$$
\left\|\widehat{\alpha}_{\tau}\left(x_{g}\right)-x_{g}\right\| \leq \sum_{\sigma \in \widehat{G}}\left\|\sigma(g) \tau(g) \widehat{\alpha}_{\tau}\left(e_{\sigma}\right) u_{g} \widehat{\alpha}_{\tau}\left(e_{\sigma}\right)-(\sigma \tau)(g) e_{\tau \sigma} u_{g} e_{\tau \sigma}\right\|<2 n \varepsilon_{0} .
$$


For $g \in G$,

$$
\begin{aligned}
\left\|x_{g} x_{g}^{*}-e\right\| & =\left\|\sum_{\sigma, \tau \in \widehat{G}} \sigma(g) \tau(g)^{-1} e_{\sigma} u_{g} e_{\sigma} e_{\tau} u_{g}^{*} e_{\tau}-\sum_{\sigma \in \widehat{G}} e_{\sigma}\right\| \\
& \leq \sum_{\sigma \in \widehat{G}}\left\|e_{\sigma} u_{g} e_{\sigma} u_{g}^{*} e_{\sigma}-e_{\sigma}\right\|<n \varepsilon_{0} .
\end{aligned}
$$

Similarly,

$$
\left\|x_{g}^{*} x_{g}-e\right\|<n \varepsilon_{0}
$$

For $a \in F \cup\left\{p_{1}\right\}$,

$$
\|e a-a e\| \leq \sum_{\tau \in \widehat{G}}\left\|e_{\tau} a-a e_{\tau}\right\|<n \varepsilon_{0} \leq \frac{1}{7} \varepsilon
$$

For $g \in G$ and $a \in F$,

$$
\begin{aligned}
\| u_{g} \text { eaeu }_{g}^{*}-x_{g} \text { eaex }_{g}^{*} \| & \leq \sum_{\sigma, \tau \in \widehat{G}}\left\|\sigma(g) \tau(g)^{-1} e_{\sigma} u_{g} e_{\sigma} a e_{\tau} u_{g}^{*} e_{\tau}-u_{g} e_{\sigma} a e_{\tau} u_{g}^{*}\right\| \\
& <9 n^{2} \varepsilon_{0}+\sum_{\sigma, \tau \in \widehat{G}}\left\|\sigma(g) \tau(g)^{-1} u_{g} a u_{g}^{*} e_{\sigma}^{2} e_{\tau}^{2}-u_{g} a u_{g}^{*} e_{\sigma} e_{\tau}\right\| \\
& =9 n^{2} \varepsilon_{0} \leq \frac{1}{7} \varepsilon
\end{aligned}
$$

For $g, h \in G$,

$$
\begin{aligned}
\left\|x_{g} x_{h}-x_{g h}\right\| & =\left\|\sum_{\sigma, \tau \in \widehat{G}} \sigma(g) \tau(h) e_{\sigma} u_{g} e_{\sigma} e_{\tau} u_{h} e_{\tau}-\sum_{\sigma \in \widehat{G}} \sigma(g h) e_{\sigma} u_{g} u_{h} e_{\sigma}\right\| \\
& \leq \sum_{\sigma \in \widehat{G}}\left\|u_{g} e_{\sigma}-e_{\sigma} u_{g}\right\|<\varepsilon_{0} n \leq \frac{1}{7} \varepsilon .
\end{aligned}
$$

For $g, h \in G$,

$$
\begin{aligned}
\left\|u_{g} x_{h} u_{g}^{*}-x_{h}\right\| & \leq \sum_{\sigma \in \widehat{G}}\left\|u_{g} e_{\sigma} u_{h} e_{\sigma} u_{g}^{*}-e_{\sigma} u_{h} e_{\sigma}\right\| \\
& \leq 2 n\left\|u_{g} e_{\sigma}-e_{\sigma} u_{g}\right\|<2 n \varepsilon_{0} \leq \frac{1}{7} \varepsilon .
\end{aligned}
$$

Now for $g \in G$ set

$$
y_{g}=\frac{1}{n} \sum_{\tau \in \widehat{G}} \widehat{\alpha}_{\tau}\left(x_{g}\right) .
$$

Then $y_{g} \in e C^{*}(G, A, \alpha) e$ and $y_{g}$ is $\widehat{\alpha}$-invariant, so $y_{g} \in e A e$. The first estimate in the previous paragraph implies that $\left\|y_{g}-x_{g}\right\|<2 n \varepsilon_{0}$. The next two then imply that

$$
\left\|y_{g} y_{g}^{*}-e\right\|<5 n \varepsilon_{0} \quad \text { and } \quad\left\|y_{g}^{*} y_{g}-e\right\|<5 n \varepsilon_{0} .
$$

Therefore there are unitaries $w_{g} \in e A e$ such that $\left\|w_{g}-y_{g}\right\|<\frac{1}{7} \varepsilon$. It follows that $\left\|w_{g}-x_{g}\right\|<\frac{1}{7} \varepsilon+2 n \varepsilon_{0} \leq \frac{2}{7} \varepsilon$. The remaining four estimates in the previous paragraph now imply, in order, Conditions (11) through (4) in Definition 3.2 For the first, this is immediate. For the second, for $g \in G$ and $a \in F$,

$$
\begin{aligned}
\left\|\alpha_{g}(e a e)-w_{g} e a e w_{g}^{*}\right\| & =\left\|u_{g} e a e u_{g}^{*}-w_{g} e a e w_{g}^{*}\right\| \\
& \leq 2\left\|w_{g}-x_{g}\right\|+\left\|u_{g} e a e u_{g}^{*}-x_{g} e a e x_{g}^{*}\right\|<\frac{5}{7} \varepsilon \leq \varepsilon .
\end{aligned}
$$

Similarly, the remaining two give $\left\|w_{g} w_{h}-w_{g h}\right\|<\varepsilon$ and $\left\|\alpha_{g}\left(w_{h}\right)-w_{h}\right\|<\frac{5}{7} \varepsilon \leq \varepsilon$. 
We next verify Condition (5) in Definition 3.2 Our choices give $1-e \precsim p_{1}$ in $C^{*}(G, A, \alpha)$. This is therefore also true in $C^{*}\left(\widehat{G}, C^{*}(G, A, \alpha), \widehat{\alpha}\right)$. Use the notation of Proposition 3.6 and the identification there of $C^{*}\left(\widehat{G}, C^{*}(G, A, \alpha), \widehat{\alpha}\right)$ with $L\left(l^{2}(G)\right) \otimes A$. By Proposition 3.6 in $L\left(l^{2}(G)\right) \otimes A$ we have

$$
\sum_{g \in G} e_{g, g} \otimes \alpha_{g}^{-1}(1-e) \precsim \sum_{g \in G} e_{g, g} \otimes \alpha_{g}^{-1}\left(p_{1}\right) .
$$

Thus, in $L\left(l^{2}(G)\right) \otimes A$ we have

$e_{1,1} \otimes(1-e) \leq \sum_{g \in G} e_{g, g} \otimes \alpha_{g}^{-1}(1-e) \precsim \sum_{g \in G} e_{g, g} \otimes \alpha_{g}^{-1}\left(p_{1}\right) \sim \sum_{g \in G} e_{1,1} \otimes p_{g} \leq e_{1,1} \otimes q$.

Therefore $1-e \precsim q$ in $A$, which verifies condition (5).

It remains to verify Condition (6). Since $\varepsilon_{0} \leq \frac{1}{7} \varepsilon$, we have $\left\|e p_{1} e\right\|>1-\frac{1}{7} \varepsilon$. Since $p_{1} \leq q$ and is nonzero, we have $\left\|p_{1}-p_{1} x p_{1}\right\|<\frac{1}{2} \varepsilon$, whence

$$
\begin{aligned}
\|e x e\| & \geq\left\|p_{1} e x e p_{1}\right\| \geq\left\|e p_{1} e\right\|-2\left\|\left[e, p_{1}\right]\right\|-\left\|p_{1}-p_{1} x p_{1}\right\| \\
& >1-\frac{1}{7} \varepsilon-\frac{2}{7} \varepsilon-\frac{1}{2} \varepsilon>1-\varepsilon .
\end{aligned}
$$

This completes the proof.

\section{Strongly tracially APPROXimately INNER AUTOMORPHiSMS}

In this section, we introduce the notion of a strongly tracially approximately inner automorphism. The main result of this section is that if an action of a finite cyclic group has the tracial Rokhlin property and is generated by a strongly tracially approximately inner automorphism, then the action is tracially approximately representable in the sense of Definition 3.2 It will follow that the dual action has the tracial Rokhlin property.

We begin by proving the "nontracial" version, essentially, that for finite cyclic groups, approximate innerness and the strict Rokhlin property imply approximate representability. This result is not particularly useful, because approximate representability seems usually to be easier to prove than the strict Rokhlin property. (See the discussion in at the beginning of Section 3.2 of [10, where approximate representability is seen primarily as a way to get actions with the strict Rokhlin property by duality.) However, the proof of the tracial version will partially follow this proof. The tracial version is much more useful, because the tracial Rokhlin property can often be proved directly.

Proposition 4.1. Let $A$ be a separable unital $\mathrm{C}^{*}$-algebra, let $\alpha \in \operatorname{Aut}(A)$ be approximately inner and satisfy $\alpha^{n}=\mathrm{id}_{A}$, and suppose that the action of $\mathbb{Z}_{n}$ generated by $\alpha$ has the strict Rokhlin property. Then the action of $\mathbb{Z}_{n}$ generated by $\alpha$ is approximately representable.

Proof. The proof has two steps. First, we show that one can choose the unitaries in the definition of approximate innerness to have order $n$, and then we show that one can in addition choose them to be $\alpha$-invariant.

Step 1: We claim that for every finite subset $F \subset A$ and every $\varepsilon>0$, there is a unitary $v \in A$ such that $\left\|v a v^{*}-\alpha(a)\right\|<\varepsilon$ for all $a \in F$, and such that $v^{n}=1$.

To prove this, without loss of generality $F$ is $\alpha$-invariant and $\|a\| \leq 1$ for all $a \in F$. Choose $\delta>0$ with

$$
\delta \leq \frac{\varepsilon}{3(2 n+7) n}
$$


and also (using semiprojectivity of $\mathbb{C}^{n}$; see Lemma 14.1.5, Theorem 14.2.1, Theorem 14.1.4, and Definition 14.1.1 of [18) so small that whenever $B$ is a unital $\mathrm{C}^{*}$-algebra and $c \in B$ satisfies

$$
\left\|c^{*} c-1\right\|<2(n-1) \delta, \quad\left\|c c^{*}-1\right\|<2(n-1) \delta, \quad \text { and } \quad\left\|c^{n}-1\right\|<2(n-1) \delta,
$$

then there is a unitary $v \in B$ such that $v^{n}=1$ and $\|v-c\|<\frac{1}{3} \varepsilon$.

Use the strict Rokhlin property to find mutually orthogonal projections $f_{0}, f_{1}, \ldots, f_{n-1} \in$ $A$ such that $\sum_{j=0}^{n-1} f_{j}=1$, such that $\left\|\alpha^{k}\left(f_{j}\right)-f_{j+k}\right\|<\delta$ for $0 \leq j, k \leq n-1$ (with the subscripts taken cyclically), and such that $\left\|\left[e_{j}, a\right]\right\|<\delta$ for $0 \leq j \leq n-1$ and $a \in F$. Then choose a unitary $u \in A$ such that whenever

$$
b \in\left\{f_{0}, f_{1}, \ldots, f_{n-1}\right\} \cup\left\{f_{j} a f_{j}: 0 \leq j \leq n-1 \text { and } a \in F\right\},
$$

we have $\left\|u b u^{*}-\alpha(b)\right\|<\delta$.

Set $w_{k}=f_{k+1} u f_{k}$ for $0 \leq k \leq n-2$, and set

$$
w_{n-1}=f_{0} u^{*} f_{1} u^{*} f_{2} \cdots f_{n-2} u^{*} f_{n-1} .
$$

Then set $w=\sum_{k=0}^{n-1} w_{k}$.

We estimate $\left\|w^{*} w-1\right\|$ and $\left\|w w^{*}-1\right\|$. First, observe that for $0 \leq k \leq n-2$ we have

$$
\begin{aligned}
\left\|w_{k}^{*} w_{k}-f_{k}\right\| & \leq\left\|u^{*} f_{k+1} u-f_{k}\right\|=\left\|f_{k+1}-u f_{k} u^{*}\right\| \\
& \leq\left\|u f_{k} u^{*}-\alpha\left(f_{k}\right)\right\|+\left\|\alpha\left(f_{k}\right)-f_{k+1}\right\|<2 \delta
\end{aligned}
$$

and similarly $\left\|w_{k}^{*} w_{k}-f_{k+1}\right\|<2 \delta$. By downwards induction on $k$, we also get

$$
\left\|\left(f_{k} u^{*} f_{k+1} \cdots f_{n-2} u^{*} f_{n-1}\right)\left(f_{k} u^{*} f_{k+1} \cdots f_{n-2} u^{*} f_{n-1}\right)^{*}-f_{k}\right\|<2(n-k-1) \delta,
$$

so that $\left\|w_{n-1} w_{n-1}^{*}-f_{0}\right\|<2(n-1) \delta$. Similarly, $\left\|w_{n-1}^{*} w_{n-1}-f_{n-1}\right\|<2(n-1) \delta$. Since the $f_{k}$ are orthogonal, we thus get

$$
\left\|w w^{*}-1\right\| \leq \max _{0 \leq k \leq n-1}\left\|w_{k}^{*} w_{k}-f_{k}\right\|<2(n-1) \delta .
$$

Similarly, $\left\|w^{*} w-1\right\|<2(n-1) \delta$.

Now we estimate $\left\|w^{n}-1\right\|$. Since the $e_{k}$ are orthogonal, we have

$$
\begin{aligned}
\| w^{n} & -1 \| \\
& =\max _{0 \leq k \leq n-1}\left\|w_{k-1} w_{k-2} \cdots w_{0} w_{n-1} \cdots w_{k}-f_{k}\right\| \\
& =\max _{0 \leq k \leq n-1}\left\|\left[f_{k} u f_{k-1} \cdots f_{1} u f_{0} u^{*} f_{1} \cdots f_{n-2} u^{*} f_{n-1} u f_{n-2} \cdots f_{k+1} u f_{k}\right]-f_{k}\right\| .
\end{aligned}
$$

Using the inequalities

$$
\left\|u f_{k} u^{*}-f_{k+1}\right\| \leq\left\|u f_{k} u^{*}-\alpha\left(f_{k}\right)\right\|+\left\|\alpha\left(f_{0}\right)-f_{k+1}\right\|<2 \delta
$$

for $0 \leq k \leq n-1$ (indices taken cyclically), and similarly $\left\|u^{*} f_{k} u-f_{k-1}\right\|<2 \delta$, one gets by induction

$$
\left\|f_{k} u f_{k-1} \cdots f_{1} u f_{0} u^{*} f_{1} \cdots f_{n-2} u^{*} f_{n-1} u f_{n-2} \cdots f_{k+1} u f_{k}-f_{k}\right\|<2(n-1) \delta
$$

for all $k$. So $\left\|w^{n}-1\right\|<2(n-1) \delta$. Thus, by the choice of $\delta$, there exists a unitary $v \in A$ such that $\|v-w\|<\frac{1}{3} \varepsilon$ and $v^{n}=1$. 
We next estimate $\left\|w a w^{*}-\alpha(a)\right\|$ for $a \in F$. Set $b=\sum_{k=0}^{n-1} f_{k} a f_{k}$ and $c=$ $\sum_{k=0}^{n-1} f_{k} \alpha(a) f_{k}$. Then

$$
\|a-b\| \leq \sum_{k=0}^{n-1} \sum_{j \neq k}\left\|f_{j} a f_{k}\right\| \leq \sum_{k=0}^{n-1} \sum_{j \neq k}\left\|\left[f_{k}, a\right]\right\|<n(n-1) \delta .
$$

Similarly, recalling that $F$ is $\alpha$-invariant, $\|\alpha(a)-c\|<n(n-1) \delta$. For $0 \leq k \leq n-2$, we have

$$
\begin{aligned}
& \left\|w_{k} f_{k} a f_{k} w_{k}^{*}-f_{k+1} \alpha(a) f_{k+1}\right\| \\
& \quad \leq\left\|f_{k+1} u f_{k} a f_{k} u^{*} f_{k+1}-f_{k+1} \alpha\left(f_{k} a f_{k}\right) f_{k+1}\right\|+2\left\|\alpha\left(f_{k}\right)-f_{k+1}\right\|<3 \delta .
\end{aligned}
$$

For $k=n-1$, we have to estimate

$$
\begin{aligned}
& \left\|w_{n-1} f_{n-1} a f_{n-1} w_{n-1}^{*}-f_{0} \alpha(a) f_{0}\right\| \\
& \quad \leq\left\|f_{n-1} a f_{n-1}-w_{n-1}^{*} f_{0} \alpha(a) f_{0} w_{n-1}\right\|+2\left\|w_{n-1} w_{n-1}^{*}-f_{0}\right\| .
\end{aligned}
$$

The last term was shown above to be less than $4(n-1) \delta$. For the first term, we claim that

$$
\left\|\left(f_{k} u f_{k-1} \cdots f_{1} u f_{0}\right) \alpha(a)\left(f_{k} u f_{k-1} \cdots f_{1} u f_{0}\right)^{*}-f_{k} \alpha^{k+1}(a) f_{k}\right\|<3 k \delta,
$$

and we prove this by induction on $k$. For $k=0$ is it trivial. If it is true for $k$, then the estimate

$$
\begin{aligned}
& \left\|f_{k+1} u f_{k} \alpha^{k+1}(a) f_{k} u^{*} f_{k+1}-f_{k+1} \alpha^{k+2}(a) f_{k+1}\right\| \\
& \quad \leq 2\left\|f_{k+1}-\alpha\left(f_{k}\right)\right\|+\left\|u f_{k} \alpha^{k+1}(a) f_{k} u^{*}-\alpha\left(f_{k} \alpha^{k+1}(a) f_{k}\right)\right\|<3 \delta .
\end{aligned}
$$

This implies that it is true for $k+1$, completing the induction. Since $\alpha^{n}=\operatorname{id}_{A}$, we get

$$
\left\|w_{n-1} f_{n-1} a f_{n-1} w_{n-1}^{*}-f_{0} \alpha(a) f_{0}\right\|<[3(n-1)+4(n-1)] \delta .
$$

By orthogonality of the summands, $\left\|w b w^{*}-c\right\|<7(n-1) \delta$, so $\left\|w a w^{*}-\alpha(a)\right\|<$ $(2 n+7) n \delta$. It follows that

$$
\left\|v a v^{*}-\alpha(a)\right\|<\frac{2}{3} \varepsilon+(2 n+7) n \delta \leq \varepsilon .
$$

This completes the proof of Step 1.

Step 2: We prove the conclusion. Let $F \subset A$ be finite, and let $\varepsilon>0$. For the same reasons as in Step 1, we may choose $\delta>0$ with

$$
\delta \leq \frac{\varepsilon}{3\left(2 n^{2}+2 n+3\right)},
$$

and also so small that whenever $B$ is a unital $\mathrm{C}^{*}$-algebra and $c \in B$ satisfies

$$
\left\|c^{*} c-1\right\|<\left(2 n^{2}+n\right) \delta, \quad\left\|c c^{*}-1\right\|<\left(2 n^{2}+n\right) \delta, \quad \text { and } \quad\left\|c^{n}-1\right\|<\left(2 n^{2}+n\right) \delta,
$$

then there is a unitary $v \in B$ such that $v^{n}=1$ and $\|v-c\|<\frac{1}{3} \varepsilon$.

Apply the result of Step 1 with $F$ as given and with $\delta$ in place of $\varepsilon$, obtaining a unitary $u$. Apply the strict Rokhlin property with $\bigcup_{k=0}^{n-1} \alpha^{k}(F \cup\{u\})$ in place of $F$ and with $\delta$ in place of $\varepsilon$, obtaining mutually orthogonal projections $f_{0}, f_{1}, \ldots, f_{n-1} \in A$.

Set $w_{k}=f_{k} \alpha^{k}(u) f_{k}$ for $0 \leq k \leq n-1$, and set $w=\sum_{k=0}^{n-1} w_{k}$. We have

$$
\left\|w_{k} w_{k}^{*}-f_{k}\right\| \leq\left\|\left[\alpha^{k}(u), f_{k}\right]\right\|<\delta,
$$


and similarly $\left\|w_{k}^{*} w_{k}-f_{k}\right\|<\delta$. Using orthogonality of the $f_{k}$, we then get

$$
\left\|w w^{*}-1\right\|=\max _{0 \leq k \leq n-1}\left\|w_{k} w_{k}^{*}-f_{k}\right\|<\delta,
$$

and similarly $\left\|w^{*} w-1\right\|<\delta$. Moreover,

$$
\left\|w_{k}^{n}-f_{k}\right\| \leq(n-1)\left\|\left[\alpha^{k}(u), f_{k}\right]\right\|<(n-1) \delta,
$$

so, using orthogonality again, $\left\|w^{n}-1\right\|<(n-1) \delta$.

Now define $y_{k}=\alpha^{k}\left(f_{0} u f_{0}\right)$ for $0 \leq k \leq n-1$, and set $y=\sum_{k=0}^{n-1} y_{k}$. Then $\left\|y_{k}-w_{k}\right\| \leq 2\left\|f_{k}-\alpha^{k}\left(f_{0}\right)\right\|<2 \delta$, so $\|y-w\|<2 n \delta$. Therefore

$$
\left\|y^{*} y-1\right\| \leq\left\|w^{*} w-1\right\|+2\|y-w\|<(2 n+1) \delta,
$$

and similarly $\left\|y y^{*}-1\right\|<(2 n+1) \delta$ and $\left\|y^{n}-1\right\|<[n \cdot 2 n+(n-1)] \delta$. We have $\alpha(y)=y$ because $\alpha^{n}=\operatorname{id}_{A}$. Apply the choice of $\delta$ with the fixed point algebra $A^{\alpha}$ in place of $B$, obtaining an $\alpha$-invariant unitary $v \in A$ such that $\|v-y\|<\frac{1}{3} \varepsilon$ and $v^{n}=1$. Then $\|v-w\|<\frac{1}{3} \varepsilon+2 n \delta$.

It remains to estimate $\left\|v a v^{*}-\alpha(a)\right\|$ for $a \in F$. Set $b=\sum_{k=0}^{n-1} f_{k} a f_{k}$ and $c=$ $\sum_{k=0}^{n-1} f_{k} \alpha(a) f_{k}$. As in the proof of Step 1, we have $\|a-b\|<n(n-1) \delta$ and $\|\alpha(a)-c\|<n(n-1) \delta$. Also,

$$
\begin{aligned}
\left\|w_{k} f_{k} a f_{k} w_{k}^{*}-f_{k} \alpha(a) f_{k}\right\| & =\left\|f_{k} \alpha^{k}(u) f_{k} a f_{k} \alpha^{k}(u)^{*} f_{k}-f_{k} \alpha(a) f_{k}\right\| \\
& =\left\|f_{k} \alpha^{k}\left(u \alpha^{-k}(a) u^{*}\right) f_{k}-f_{k} \alpha(a) f_{k}\right\|+2\left\|\left[\alpha^{k}(u), f_{k}\right]\right\| \\
& \leq\left\|u \alpha^{-k}(a) u^{*}-\alpha^{-k+1}(a)\right\|+2\left\|\left[\alpha^{k}(u), f_{k}\right]\right\|<3 \delta .
\end{aligned}
$$

Using orthogonality of the $f_{k}$, we then get $\left\|w b w^{*}-c\right\|<3 \delta$, whence $\left\|w a w^{*}-\alpha(a)\right\|<$ $[2 n(n-1)+3] \delta$. Therefore

$$
\left\|v a v^{*}-\alpha(a)\right\|<2\left(\frac{1}{3} \varepsilon+2 n \delta\right)+[2 n(n-1)+3] \delta \leq \varepsilon .
$$

as was to be proved.

We now define a strongly tracially approximately inner automorphism. We call the condition "strong tracial approximate innerness" because there is a different condition, Definition [5.1] which is more appropriately called tracial approximate innerness. In particular, it is unlikely to be true that the product of two automorphisms which are strongly tracially approximately inner as defined here is again strongly tracially approximately inner. We have not been able to prove the results of this section with tracial approximate innerness in place of strong tracial approximate innerness. However, the tracially approximately inner automorphisms form a group (Theorem [5.8), and, moreover, if $A$ is an infinite dimensional simple separable unital $\mathrm{C}^{*}$-algebra with tracial rank zero, then a tracially approximately inner automorphism of finite order is necessarily strongly tracially approximately inner (combine Proposition 6.2 and Theorem 6.6).

Definition 4.2. Let $A$ be an infinite dimensional simple separable unital $\mathrm{C}^{*}$ algebra and let $\alpha \in \operatorname{Aut}(A)$. We say that $\alpha$ is strongly tracially approximately inner if for every finite set $F \subset A$, every $\varepsilon>0$, and every positive element $x \in A$ with $\|x\|=1$, there exist a projection $e \in A$ and a unitary $v \in e A e$ such that:

(1) $\|\alpha(e)-e\|<\varepsilon$.

(2) $\|e a-a e\|<\varepsilon$ for all $a \in F$.

(3) $\|$ veaev $^{*}-\alpha($ eae $) \|<\varepsilon$ for all $a \in F$.

(4) $1-e$ is Murray-von Neumann equivalent to a projection in $\overline{x A x}$. 
(5) $\|$ exe $\|>1-\varepsilon$.

Our original definition, in [22], had a condition like that in Remark 1.3] in place of (15).

As in Definition 1.2 we allow $e=1$, in which case conditions (4) and (5) are vacuous.

Remark 4.3. Let $A$ be an infinite dimensional simple separable unital $\mathrm{C}^{*}$-algebra and let $\alpha \in \operatorname{Aut}(A)$. If $\alpha$ is approximately inner then $\alpha$ is strongly tracially approximately inner. If $\alpha$ is strongly tracially approximately inner and $A$ does not have Property (SP), then $\alpha$ is approximately inner.

Example 2.9 of [24] shows that a strongly tracially approximately inner automorphism need not be approximately inner, even on a simple AF algebra. In fact, in Theorem 6.6. we give a condition which implies that many automorphisms which are not inner are nevertheless strongly tracially approximately inner.

When $A$ is finite, we do not need Condition (5) of Definition 4.2.

Lemma 4.4. Let $A$ be an infinite dimensional finite simple separable unital $\mathrm{C}^{*}$ algebra and let $\alpha \in \operatorname{Aut}(A)$. Then $\alpha$ is strongly tracially approximately inner if and only if for every finite set $F \subset A$, every $\varepsilon>0$, and every positive element $x \in A$ with $\|x\|=1$, there exist a projection $e \in A$ and a unitary $v \in e A e$ such that:

(1) $\|\alpha(e)-e\|<\varepsilon$.

(2) $\|e a-a e\|<\varepsilon$ for all $a \in F$.

(3) $\|$ veaev $^{*}-\alpha(e a e) \|<\varepsilon$ for all $a \in F$.

(4) $1-e$ is Murray-von Neumann equivalent to a projection in $\overline{x A x}$.

Proof. The proof is the same as for Lemma 1.16

We now prove the tracial analog of Proposition 4.1. We separate the analog of Step 1 of its proof as a separate lemma.

Lemma 4.5. Let $A$ be an infinite dimensional simple separable unital $\mathrm{C}^{*}$-algebra with Property (SP), and let $\alpha \in \operatorname{Aut}(A)$ be strongly tracially approximately inner and satisfy $\alpha^{n}=\operatorname{id}_{A}$. Suppose $\alpha$ generates an action of $\mathbb{Z}_{n}$ with the tracial Rokhlin property. Then for every finite set $F \subset A$, every $\varepsilon>0$, and every positive element $x \in A$ with $\|x\|=1$, there exist a projection $e \in A$ and a unitary $v \in e A e$ such that:

(1) $\alpha(e)=e$ and $v^{n}=e$.

(2) $\|e a-a e\|<\varepsilon$ for all $a \in F$.

(3) $\|$ veaev $^{*}-\alpha(e a e) \|<\varepsilon$ for all $a \in F$.

(4) $1-e$ is Murray-von Neumann equivalent to a projection in $\overline{x A x}$.

(5) $\|$ exe $\|>1-\varepsilon$.

Proof. Let $F \subset A$ be finite, let $\varepsilon>0$, and let $x \in A$ be a positive element with $\|x\|=1$. Without loss of generality $\|a\| \leq 1$ for all $a \in F$.

Set

$$
\rho=\min \left(\frac{1}{n}, \frac{1}{5}\right) \quad \text { and } \quad \varepsilon_{0}=\frac{\varepsilon}{168 n^{2}} .
$$

Choose $\varepsilon_{1}>0$ with

$$
\varepsilon_{1} \leq \min \left(\frac{1}{36}, \varepsilon_{0}\right)
$$

and so small that the following are all true in any $\mathrm{C}^{*}$-algebra $B$ : 
- Whenever $r_{1}, r_{2} \in B$ are projections, and $c \in B$ satisfies $\left\|c^{*} c-r_{1}\right\|<27 \varepsilon_{1}$ and $\left\|c c^{*}-r_{2}\right\|<27 \varepsilon_{1}$, then there is $v \in B$ such that $\|v-c\|<\varepsilon_{0}, v^{*} v=r_{1}$, and $v v^{*}=r_{2}$.

- Whenever $e, q \in B$ are projections such that $\|e q-q e\|<\varepsilon_{1}$, then there exists a projection $r \leq q$ such that $\|r-e q\|<\min \left(\frac{1}{2}, \frac{1}{12} \varepsilon\right)$.

- Whenever $e_{0}, e_{1}, \ldots, e_{n-1}$ and $f_{0}, f_{1}, \ldots, f_{n-1}$ are two sets of mutually orthogonal projections in $B$ such that $\left\|e_{j} f_{j}-e_{j}\right\|<3 \varepsilon_{1}$ for $0 \leq j \leq n-1$, then there exist mutually orthogonal projections $h_{0}, h_{1}, \ldots, h_{n-1} \in B$ such that $h_{j} \geq e_{j}$ and $\left\|h_{j}-f_{j}\right\|<\rho$ for $0 \leq j \leq n-1$.

Choose $\varepsilon_{2}>0$ so small that if $r_{0}, \ldots, r_{n-1}$ are projections in a $\mathrm{C}^{*}$-algebra $B$ such that $\left\|r_{j} r_{k}\right\|<3 \varepsilon_{2}$ for $j \neq k$, then there is a projection $z$ in the $\mathrm{C}^{*}$-subalgebra of $B$ generated by $\sum_{j=0}^{n-1} r_{j}$, and there are mutually orthogonal projections $z_{j} \in B$, such that $\sum_{j=0}^{n-1} z_{j}=z$, and such that $\left\|z_{j}-r_{j}\right\|<\varepsilon_{1}$ for $0 \leq j \leq n-1$. We also require $\varepsilon_{2} \leq \min \left(\frac{1}{6}, \varepsilon_{1}\right)$.

Choose $\varepsilon_{3}>0$ so small that whenever $B$ is a $\mathrm{C}^{*}$-algebra and $e, q \in B$ are projections such that $\|e q-q e\|<\varepsilon_{3}$, then there exists a projection $r \leq q$ such that $\|r-e q\|<\varepsilon_{2}$. We also require $\varepsilon_{3} \leq \min \left(\varepsilon_{2}, 1 / n^{2}\right)$.

Apply Lemma 1.14 obtaining a nonzero projection $q \in \overline{x A x}$ such that:

(1) $\|r x r-r\|<\varepsilon_{1}$ for every nonzero projection $r \leq q$.

Using Lemma 1.10 find nonzero projections $q_{0}, q_{1}, \ldots, q_{n-1}, g \in A$ such that $g+$ $\sum_{j=0}^{n-1} q_{j}=q$. Lemma 3.10 provides a nonzero projection $g_{0} \leq g$ such that $\alpha^{j}\left(g_{0}\right) \precsim$ $q_{j}$ for $0 \leq j \leq n-1$.

Set $F_{1}=\bigcup_{j=0}^{n-1} \alpha^{j}\left(F \cup\left\{g_{0}\right\}\right)$. By the tracial Rokhlin property (Definition [1.2), there exist orthogonal projections $f_{0}, f_{1}, \ldots, f_{n-1} \in A$ such that, with $f=\sum_{j=0}^{n-1} f_{j}$, we have:

(2) With indices taken $\bmod n$, we have $\left\|\alpha^{m}\left(f_{j}\right)-f_{j+m}\right\|<\varepsilon_{3}$ for $0 \leq j, m \leq$ $n-1$.

(3) $\left\|f_{j} a-a f_{j}\right\|<\varepsilon_{3}$ for $0 \leq j \leq n-1$ and all $a \in F_{1}$.

(4) $1-f$ is Murray-von Neumann equivalent to a projection in $g_{0} A g_{0}$.

(5) $\left\|f g_{0} f\right\|>1-\varepsilon_{3}$.

For $j \neq k$ we have $\left\|f_{j} g_{0} f_{k}\right\|<\varepsilon_{3}$, so

$$
\begin{aligned}
\max _{0 \leq j \leq n-1}\left\|f_{j} g_{0} f_{j}\right\| & =\left\|\sum_{j=0}^{n-1} f_{j} g_{0} f_{j}\right\| \\
& >\left\|f g_{0} f\right\|-n(n-1) \varepsilon_{3}>1-\varepsilon_{3}-n(n-1) \varepsilon_{3}>1-n^{2} \varepsilon_{3} .
\end{aligned}
$$

Therefore there is $j$ such that $\left\|f_{j} g_{0} f_{j}\right\|>1-n^{2} \varepsilon_{3}$. Since all the other conditions on the $f_{j}$ are invariant under cyclic permutation of the indices, without loss of generality $\left\|f_{0} g_{0} f_{0}\right\|>1-n^{2} \varepsilon_{3}$.

By the choice of $\varepsilon_{3}$, there are projections $r_{1} \leq g_{0}$ and $r_{2} \leq f_{0}$ such that $\| r_{1}-$ $f_{0} g_{0} \|<\varepsilon_{2}$ and $\left\|r_{2}-f_{0} g_{0}\right\|<\varepsilon_{2}$. In particular:

(6) $\left\|r_{1}-r_{2}\right\|<2 \varepsilon_{2}$.

Further, using $\left\|f_{0} g_{0}-g_{0} f_{0}\right\|<\varepsilon_{3} \leq \varepsilon_{2}$, we get $\left\|r_{1}-f_{0} g_{0} f_{0}\right\|<2 \varepsilon_{2}$. Since $\left\|f_{0} g_{0} f_{0}\right\|>$ $1-n^{2} \varepsilon_{3}$ and $3 \varepsilon_{2}+n^{2} \varepsilon_{3}<1$, it follows that $r_{1} \neq 0$.

Set

$$
F_{2}=F_{1} \cup\left\{r_{1}\right\} \cup\left\{\alpha^{j}\left(f_{k}\right): 1 \leq j, k \leq n-1\right\}
$$


Apply strong tracial approximate innerness (Definition 4.2), obtaining a projection $p_{0} \in A$ and a partial isometry $w \in A$ such that:

(7) $w^{*} w=p$ and $w w^{*}=\alpha(p)$.

(8) $\|p a-a p\|<\varepsilon_{3}$ for all $a \in F_{2}$.

(9) $\|$ wpapw* $w^{*} \alpha($ pap $) \|<\varepsilon_{3}$ for all $a \in F_{2}$.

(10) $1-p \precsim r_{1}$.

(11) $\left\|p r_{1} p\right\|>1-\varepsilon_{3}$.

By the choice of $\varepsilon_{3}$, there are projections $s_{1} \leq f_{0}$ and $s_{2} \leq p$ such that:

(12) $\left\|s_{1}-f_{0} p\right\|<\varepsilon_{2}$ and $\left\|s_{2}-f_{0} p\right\|<\varepsilon_{2}$.

Then also:

(13) $\left\|s_{1}-f_{0} p f_{0}\right\|<\varepsilon_{2}$.

(14) $\left\|s_{2}-p f_{0} p\right\|<\varepsilon_{2}$.

(15) $\left\|s_{1}-s_{2}\right\|<2 \varepsilon_{2}$.

For $0 \leq j<k \leq n-1$, we have, using (13) at the third step, $f_{0} f_{k-j}=0$ at the fourth step, and (2) and $\varepsilon_{3} \leq \varepsilon_{2}$ at the last step,

$$
\begin{aligned}
\left\|\alpha^{j}\left(s_{1}\right) \alpha^{k}\left(s_{1}\right)\right\| & =\left\|s_{1} \alpha^{k-j}\left(s_{1}\right)\right\| \\
& \leq\left\|f_{0} p f_{0} \alpha^{k-j}\left(f_{0}\right) \alpha^{k-j}(p) \alpha^{k-j}\left(f_{0}\right)\right\|+2\left\|s_{1}-f_{0} p f_{0}\right\| \\
& <\left\|f_{0} \alpha^{k-j}\left(f_{0}\right)\right\|+2 \varepsilon_{2} \leq\left\|f_{0}-\alpha^{k-j}\left(f_{0}\right)\right\|+2 \varepsilon_{2}<3 \varepsilon_{2} .
\end{aligned}
$$

By the choice of $\varepsilon_{2}$, there are projections $e_{0}, e_{1}, \ldots, e_{n-1} \in A$ such that the sum $e=\sum_{j=0}^{n-1} e_{j}$ is in the $\mathrm{C}^{*}$-algebra generated by $\sum_{j=0}^{n-1} \alpha^{j}\left(s_{1}\right)$ (and, in particular, is $\alpha$-invariant), and such that:

(16) $\left\|e_{j}-\alpha^{j}\left(s_{1}\right)\right\|<\varepsilon_{1}$ for $0 \leq j \leq n-1$.

We then get:

(17) $\left\|\alpha^{m}\left(e_{j}\right)-e_{j+m}\right\|<2 \varepsilon_{1}$, with indices taken $\bmod n$, for $0 \leq j, k \leq n-1$.

Define $c_{j}=e_{j+1} \alpha^{j}(w) e_{j}$ for $0 \leq j \leq n-2$. (We can't use $e_{j+1} w e_{j}$, imitating the proof of Step 1 of Proposition 4.1] because we don't know $e_{j}$ and $e_{j+1}$ are even approximately dominated by $p$ and $\alpha(p)$.) We claim that

$$
\left\|c_{j}^{*} c_{j}-e_{j}\right\|<27 \varepsilon_{1} \quad \text { and } \quad\left\|c_{j} c_{j}^{*}-e_{j+1}\right\|<27 \varepsilon_{1}
$$

for $0 \leq j \leq n-2$. We start by observing that (16), (15), and (14) imply

$$
\left\|e_{0}-p f_{0} p\right\| \leq\left\|e_{0}-s_{1}\right\|+\left\|s_{1}-s_{2}\right\|+\left\|s_{2}-p f_{0} p\right\|<\varepsilon_{1}+2 \varepsilon_{2}+\varepsilon_{2} \leq 4 \varepsilon_{1} .
$$

So (9) implies

$$
\left\|w e_{0} w^{*}-\alpha\left(e_{0}\right)\right\|<8 \varepsilon_{1}+\left\|w p f_{0} p w^{*}-\alpha\left(p f_{0} p\right)\right\|<8 \varepsilon_{1}+\varepsilon_{3} \leq 9 \varepsilon_{1} .
$$

Combining this with $\left\|\alpha\left(e_{0}\right)-e_{1}\right\|<2 \varepsilon_{1}$ (from (17)), we get:

(18) $\left\|w e_{0} w^{*}-e_{1}\right\|<11 \varepsilon_{1}$.

Therefore also $\left\|e_{1} w^{*} e_{0} w e_{1}-e_{1}\right\|<11 \varepsilon_{1}$. Furthermore, using $w^{*} w=p$ at the first step, $s_{2} \leq p$ at the second step, and (15) and (16) at the third step,

$$
\begin{aligned}
\left\|w^{*} e_{1} w-e_{0}\right\| & \leq\left\|w^{*}\right\| \cdot\left\|e_{1}-w e_{0} w^{*}\right\| \cdot\|w\|+\left\|p e_{0} p-e_{0}\right\| \\
& \leq\left\|e_{1}-w e_{0} w^{*}\right\|+2\left(\left\|s_{2}-s_{1}\right\|+\left\|s_{1}-e_{0}\right\|\right) \\
& <11 \varepsilon_{1}+4 \varepsilon_{2}+2 \varepsilon_{1} \leq 17 \varepsilon_{1} .
\end{aligned}
$$

So $\left\|e_{0} w^{*} e_{1} w e_{0}-e_{0}\right\|<17 \varepsilon_{1}$. This does the case $j=0$ of the claim, with $17 \varepsilon_{1}$ in place of $27 \varepsilon_{1}$. For the general case, use (17) and the definition of $c_{j}$ to get: 
(19) $\left\|c_{j}-\alpha^{j}\left(c_{0}\right)\right\|<4 \varepsilon_{1}$.

Use this and (17) again to get

$$
\left\|c_{j}^{*} c_{j}-e_{j}\right\|<10 \varepsilon_{1}+\left\|c_{0}^{*} c_{0}-e_{0}\right\|<27 \varepsilon_{1}
$$

and, similarly, $\left\|c_{j} c_{j}^{*}-e_{j+1}\right\|<27 \varepsilon_{1}$. This proves the claim.

By the choice of $\varepsilon_{1}$, there are partial isometries $v_{j} \in B$ such that:

(20) $\left\|v_{j}-c_{j}\right\|<\varepsilon_{0}, v_{j}^{*} v_{j}=e_{j}$, and $v_{j} v_{j}^{*}=e_{j+1}$ for $0 \leq j \leq n-2$.

Define $v=v_{0}^{*} v_{1}^{*} \cdots v_{n-2}^{*}+\sum_{j=0}^{n-2} v_{j}$. Then it is easily checked that $v$ is a unitary in $e A e$ which satisfies $v^{n}=1$.

We now have $e$ and $v$, and Part (11) of the conclusion has been verified.

For Part (21), if $a \in F$ and $0 \leq j \leq n-1$, then $\alpha^{-j}(a) \in F_{1} \subset F_{2}$, so, using (17), (16), (12), (8), and (3),

$$
\begin{aligned}
\left\|e_{j} a-a e_{j}\right\| \leq & 2\left\|e_{j}-\alpha^{j}\left(e_{0}\right)\right\|+\left\|e_{0} \alpha^{-j}(a)-\alpha^{-j}(a) e_{0}\right\| \\
\leq & 2\left\|e_{j}-\alpha^{j}\left(e_{0}\right)\right\|+2\left\|e_{0}-s_{1}\right\| \\
& \quad+2\left\|s_{1}-f_{0} p\right\|+\|p a-a p\|+\left\|f_{0} a-a f_{0}\right\| \\
& <4 \varepsilon_{1}+2 \varepsilon_{1}+2 \varepsilon_{2}+\varepsilon_{3}+\varepsilon_{3} \leq 10 \varepsilon_{1} .
\end{aligned}
$$

Since $e=\sum_{j=0}^{n-1} e_{j}$, it follows that $\|e a-a e\|<10 n \varepsilon_{1} \leq \varepsilon$. This is Part (2) of the conclusion. The relation $\left\|e_{j} a-a e_{j}\right\|<10 \varepsilon_{1}$ furthermore implies that:

(21) $\left\|e a e-\sum_{j=0}^{n-1} e_{j} a e_{j}\right\|<10 n(n-1) \varepsilon_{1}$.

To start (3i), we estimate $\left\|v_{j} e_{j} a e_{j} v_{j}^{*}-\alpha\left(e_{j} a e_{j}\right)\right\|$ for $a \in F$ and $0 \leq j \leq n-2$. We begin with $j=0$ and $a \in \bigcup_{j=0}^{n-1} \alpha^{-j}(F) \subset F_{2}$. Use (16), (15), and $s_{2} \leq p$ to get

$$
\left\|e_{0} p-e_{0}\right\| \leq 2\left\|e_{0}-s_{1}\right\|+2\left\|s_{1}-s_{2}\right\|<2 \varepsilon_{1}+4 \varepsilon_{2} \leq 6 \varepsilon_{1},
$$

and then use $w^{*} w=p$ and (18) to get

$$
\left\|e_{1} w-w e_{0}\right\| \leq\left\|e_{1}-w e_{0} w^{*}\right\| \cdot\|w\|+\left\|e_{0} p-e_{0}\right\|<11 \varepsilon_{1}+6 \varepsilon_{1}=17 \varepsilon_{1} .
$$

Next, use the definition of $c_{0}$ in the first step and (17) and (9) in the last step to get

$$
\begin{aligned}
\left\|c_{0} e_{0} a e_{0} c_{0}^{*}-\alpha\left(e_{0} a e_{0}\right)\right\| \leq & 4\left\|e_{0}-e_{0} p\right\|+\| e_{1} \text { we }_{0} \text { pape }_{0} w^{*} e_{1}-\alpha\left(e_{0} \text { pape }_{0}\right) \| \\
\leq & 4\left\|e_{0}-e_{0} p\right\|+2 \| e_{1} \text { we }_{0}-\text { we }_{0} \| \\
& \quad+2\left\|\alpha\left(e_{0}\right)-e_{1}\right\|+\| e_{1}\left[\text { wpapw }^{*}-\alpha(\text { pap })\right] e_{1} \| \\
& <24 \varepsilon_{1}+34 \varepsilon_{1}+4 \varepsilon_{1}+\varepsilon_{3} \leq 63 \varepsilon_{1} .
\end{aligned}
$$

Using (20) and $\varepsilon_{1} \leq \varepsilon_{0}$, we get $\left\|v_{0} e_{0} a e_{0} v_{0}^{*}-\alpha\left(e_{0} a e_{0}\right)\right\|<65 \varepsilon_{0}$.

Now, for $a \in F$ and $0 \leq j \leq n-2$, combine

$$
\left\|c_{0} e_{0} \alpha^{-j}(a) e_{0} c_{0}^{*}-\alpha\left(e_{0} \alpha^{-j}(a) e_{0}\right)\right\|<63 \varepsilon_{1}
$$

with (17) and (19) to get

$$
\left\|c_{j} e_{j} a e_{j} c_{j}^{*}-\alpha\left(e_{j} a e_{j}\right)\right\|<63 \varepsilon_{1}+2\left\|c_{j}-\alpha^{j}\left(c_{0}\right)\right\|+4\left\|e_{j}-\alpha^{j}\left(e_{0}\right)\right\|<79 \varepsilon_{1} .
$$

Using (20) and $\varepsilon_{1} \leq \varepsilon_{0}$, we then get

(22) $\left\|v_{j} e_{j} a e_{j} v_{j}^{*}-\alpha\left(e_{j} a e_{j}\right)\right\|<81 \varepsilon_{0}$ for $0 \leq j \leq n-2$ and $a \in \bigcup_{j=0}^{n-1} \alpha^{j}(F)$. 
Set

$$
y_{k}=\alpha^{k-1}\left(v_{0}\right) \alpha^{k-2}\left(v_{0}\right) \cdots \alpha\left(v_{0}\right) v_{0} .
$$

An induction argument gives $\left\|\alpha^{k}\left(e_{0} a e_{0}\right)-y_{k} e_{0} a e_{0} y_{k}^{*}\right\|<81 k \varepsilon_{0}$ for $k \geq 0$ and $a \in \bigcup_{j=0}^{n-1} \alpha^{j}(F)$. The inequalities (19) and (20) give $\left\|\alpha^{j}\left(v_{0}\right)-v_{j}\right\|<4 \varepsilon_{1}+2 \varepsilon_{0} \leq 6 \varepsilon_{0}$. Therefore, with $z=v_{0}^{*} v_{1}^{*} \cdots v_{n-2}^{*}$, we get

$$
\left\|\alpha^{n-1}\left(e_{0} a e_{0}\right)-z^{*} e_{0} a e_{0} z\right\|<81(n-1) \varepsilon_{0}+6(n-1) \varepsilon_{0}=87(n-1) \varepsilon_{0} .
$$

Putting $\alpha(a)$ in place of $a$, using $\alpha^{n}=\operatorname{id}_{A}$, and rearranging, we get

$$
\left\|z \alpha^{n-1}\left(e_{0}\right) a \alpha^{n-1}\left(e_{0}\right) z^{*}-\alpha\left(\alpha^{n-1}\left(e_{0}\right) a \alpha^{n-1}\left(e_{0}\right)\right)\right\|<87(n-1) \varepsilon_{0} .
$$

From (17) and $\varepsilon_{1} \leq \varepsilon_{0}$, it follows that

$$
\left\|z e_{n-1} a e_{n-1} z^{*}-\alpha\left(e_{n-1} a e_{n-1}\right)\right\|<[87(n-1)+8] \varepsilon_{0} .
$$

Using the definition of $v$ and (22), we now get

$\left\|v\left(\sum_{j=0}^{n-1} e_{j} a e_{j}\right) v^{*}-\alpha\left(\sum_{j=0}^{n-1} e_{j} a e_{j}\right)\right\|<81(n-1) \varepsilon_{0}+[87(n-1)+8] \varepsilon_{0} \leq 168 n \varepsilon_{0}$.

From (21) it now follows that

$$
\| \text { veaev }^{*}-\alpha(\text { eae }) \|<168 n \varepsilon_{0}+20 n(n-1) \varepsilon_{1} \leq 168 n^{2} \varepsilon_{0} \leq \varepsilon .
$$

This completes the proof of Part (3) of the conclusion.

For Part (44), begin by using (16), (21), and $s_{1} f_{0}=s_{1}$ to estimate

$$
\left\|e_{j} f_{j}-e_{j}\right\| \leq 2\left\|e_{j}-\alpha^{j}\left(s_{1}\right)\right\|+\left\|f_{j}-\alpha^{j}\left(f_{0}\right)\right\|<2 \varepsilon_{1}+\varepsilon_{3} \leq 3 \varepsilon_{1} .
$$

The conditions on $\varepsilon_{1}$ provide mutually orthogonal projections $h_{j} \geq e_{j}$ such that $\left\|h_{j}-f_{j}\right\|<\rho$. Set $h=\sum_{j=0}^{n-1} h_{j}$. Then $1-e=1-h+\sum_{j=0}^{n-1}\left(h_{j}-e_{j}\right)$. Since $\|h-f\|<n \rho \leq 1$, it follows (using (4) at the second step) that

$$
1-h \sim 1-f \precsim g_{0} \leq g .
$$

The next step is to show that $h_{0}-e_{0} \precsim 1-p$. We have, using (12), (15), (16), and $s_{2} \leq p$ at the third step,

$$
\begin{aligned}
\left\|(1-p)\left(h_{0}-e_{0}\right)-\left(h_{0}-e_{0}\right)\right\| & =\left\|p h_{0}-p e_{0}\right\| \\
& \leq\left\|h_{0}-f_{0}\right\|+\left\|p f_{0}-p s_{2}\right\|+\left\|s_{2}-s_{1}\right\|+\left\|s_{1}-e_{0}\right\| \\
& <\rho+\varepsilon_{2}+2 \varepsilon_{2}+\varepsilon_{1}<1 .
\end{aligned}
$$

So $h_{0}-e_{0} \precsim 1-p$ by Lemma 2.5.2 of [16].

Next, using (2), for $0 \leq j \leq n-1$ we have

$$
\left\|h_{j}-\alpha^{j}\left(h_{0}\right)\right\| \leq\left\|h_{j}-f_{j}\right\|+\left\|h_{0}-f_{0}\right\|+\left\|f_{j}-\alpha^{j}\left(f_{0}\right)\right\|<\rho+\rho+\varepsilon_{3},
$$

and so, using (17) and $\varepsilon_{3} \leq \varepsilon_{1}$,

$$
\left\|\left(h_{j}-e_{j}\right)-\alpha^{j}\left(h_{0}-e_{0}\right)\right\|<2 \rho+3 \varepsilon_{1} \leq 1 .
$$

Thus, using (10) at the third step,

$$
h_{j}-e_{j} \sim \alpha^{j}\left(h_{0}-e_{0}\right) \precsim \alpha^{j}(1-p) \precsim \alpha^{j}\left(r_{1}\right) \leq \alpha^{j}\left(g_{0}\right) \precsim q_{j} .
$$

We conclude that

$$
1-e=1-h+\sum_{j=0}^{n-1}\left(h_{j}-e_{j}\right) \precsim g+\sum_{j=0}^{n-1} q_{j} \leq q \in \overline{x A x} .
$$

This proves Part (4). 
It remains to prove Part (51) of the conclusion. Combine (12) and (16) to get $\left\|e_{0}-f_{0} p\right\|<\varepsilon_{2}+\varepsilon_{1} \leq 2 \varepsilon_{1}$. Use (6) at the second and third steps, $r_{2} \leq f_{0}$ at the second step, and (11) at the fourth step, to get

$$
\begin{aligned}
\left\|e_{0} r_{1} e_{0}\right\| & >\left\|p f_{0} r_{1} f_{0} p\right\|-4 \varepsilon_{1}>\left\|p r_{2} p\right\|-2 \varepsilon_{2}-4 \varepsilon_{1}>\left\|p r_{1} p\right\|-4 \varepsilon_{2}-4 \varepsilon_{1} \\
& >1-\varepsilon_{3}-4 \varepsilon_{2}-4 \varepsilon_{1} \geq 1-9 \varepsilon_{1} .
\end{aligned}
$$

Since $1-9 \varepsilon_{1} \geq \frac{1}{4}$, we get in particular $\left\|e_{0} r_{1}\right\|>\frac{1}{2}$.

Next, we estimate $\left\|e_{0} r_{1}-r_{1} e_{0}\right\|$. We have $\left\|p r_{1}-r_{1} p\right\|<\varepsilon_{3}$ by (8), and $\| f_{0} r_{1}-$ $r_{1} f_{0}\|\leq 2\| r_{1}-r_{2} \|<4 \varepsilon_{2}$ by (6) and because $r_{2} \leq f_{0}$. So

$\left\|e_{0} r_{1}-r_{1} e_{0}\right\| \leq 2\left\|e_{0}-f_{0} p\right\|+\left\|f_{0} r_{1}-r_{1} f_{0}\right\|+\left\|p r_{1}-r_{1} p\right\|<4 \varepsilon_{1}+4 \varepsilon_{2}+\varepsilon_{3} \leq 9 \varepsilon_{1}$.

It follows from the choice of $\varepsilon_{1}$ that there is a projection $r \leq e_{0}$ such that $\| r-$ $e_{0} r_{1} \|<\min \left(\frac{1}{2}, \frac{1}{12} \varepsilon\right)$. Since $\left\|e_{0} r_{1}\right\|>\frac{1}{2}$, we have $r \neq 0$.

Now use $r \leq e_{0} \leq e$ at the first step, and $r_{1} \leq g_{0} \leq q$ and (11) at the third step, to get

$\|$ exe $\|\geq\| r x r\|>\| e_{0} r_{1} x r_{1} e_{0}\left\|-\frac{1}{6} \varepsilon>\right\| e_{0} r_{1} e_{0} \|-\varepsilon_{1}-\frac{1}{6} \varepsilon>1-9 \varepsilon_{1}-\varepsilon_{1}-\frac{1}{6} \varepsilon \geq 1-\varepsilon$.

This completes the proof.

Theorem 4.6. Let $A$ be an infinite dimensional simple separable unital $\mathrm{C}^{*}$-algebra and let $\alpha \in \operatorname{Aut}(A)$ be strongly tracially approximately inner and satisfy $\alpha^{n}=\operatorname{id}_{A}$. Suppose $\alpha$ generates an action of $\mathbb{Z}_{n}$ with the tracial Rokhlin property. Then this action is tracially approximately representable in the sense of Definition 3.2

Proof. If $A$ does not have Property (SP), then $\alpha$ is approximately inner (by Remark 4.3) and the action of $\mathbb{Z}_{n}$ it generates has the strict Rokhlin property (by Lemma 1.13), so the result is Proposition 4.1. Accordingly, we assume that $A$ has Property (SP). Let $F \subset A$ be finite, let $\varepsilon>0$, and let $x \in A$ be a positive element with $\|x\|=1$.

Choose $\varepsilon_{1}>0$ with

$$
\varepsilon_{1} \leq \min \left(\frac{1}{n}, \frac{\varepsilon}{3 n\left(2 n^{2}+2 n+3\right)}, \frac{\varepsilon}{2(n+5)}\right),
$$

and also (using semiprojectivity of $\mathbb{C}^{n}$, as at the beginning of the proof of Proposition (4.1) so small that whenever $B$ is a unital $\mathrm{C}^{*}$-algebra and $c \in B$ satisfies

$$
\left\|c^{*} c-1\right\|<2(n-1) \varepsilon_{1}, \quad\left\|c c^{*}-1\right\|<2(n-1) \varepsilon_{1}, \quad \text { and } \quad\left\|c^{n}-1\right\|<2(n-1) \varepsilon_{1},
$$

then there is a unitary $v \in B$ such that $v^{n}=1$ and $\|v-c\|<\varepsilon /(3 n)$. Choose $\varepsilon_{2}>0$ with $\varepsilon_{2} \leq \frac{1}{8} \varepsilon_{1}$, and also so small that whenever $B$ is a unital $\mathrm{C}^{*}$-algebra, $C \subset B$ is a subalgebra, $p \in B$ is a projection, and $b \in C$ satisfies $\|b-p\|<3 n^{2} \varepsilon_{2}$, then there is a projection $q \in C$ and a unitary $z \in B$ such that $z p z^{*}=q$ and $\|z-1\|<\frac{1}{8} \varepsilon_{1}$. Choose $\varepsilon_{3}>0$ with $\varepsilon_{3} \leq \min \left(\frac{1}{2}, \varepsilon_{2}\right)$, and also so small that whenever $B$ is a unital $\mathrm{C}^{*}$-algebra, $f_{0}, f_{1}, \ldots, f_{n-1} \in B$ are mutually orthogonal projections, and $e \in B$ is a projection such that $\left\|\left[f_{j}, e\right]\right\|<\varepsilon_{3}$ for $0 \leq j \leq n-1$, then there exist mutually orthogonal projections $g_{0}, g_{1}, \ldots, g_{n-1} \leq e$ such that $\left\|g_{j}-f_{j} e\right\|<\varepsilon_{2}$ for $0 \leq j \leq n-1$.

By considering polynomial approximations to the continuous functional calculus, choose $\delta>0$ with $\delta \leq \frac{1}{6} \varepsilon$, and also so small that whenever $B$ is a unital $\mathrm{C}^{*}$-algebra, $x \in B$ satisfies $0 \leq x \leq 1$, and $q \in B$ is a projection such that $\|[x, q]\|<\delta$, then $\left\|\left[x^{1 / 2}, q\right]\right\|<\frac{1}{6} \varepsilon$. Apply Lemma 1.14 to find a nonzero projection $q_{0} \in \overline{x A x}$ such 
that whenever $q \leq q_{0}$ is a nonzero projection, then $\|q x q-q\|<\delta$ and $\|[x, q]\|<\delta$. Use Lemma 1.10 to choose orthogonal nonzero projections $q_{1}, q_{2} \leq q$.

Apply Lemma 4.5 with $\bigcup_{k=0}^{n-1} \alpha^{k}(F)$ in place of $F$, with $\varepsilon_{3}$ in place of $\varepsilon$, and with $q_{1}$ in place of $x$. Call the resulting projection $e$, and let $u \in e A e$ be the resulting unitary.

Set $\lambda=\left\|e q_{1} e\right\|>1-\varepsilon_{3}$, and set $b=\lambda^{-1} e q_{1} e$. Thus $\left\|b-e q_{1} e\right\|=\lambda^{-1}-1<2 \varepsilon_{3}$. Apply Lemma 1.14 to find a nonzero projection $r \in \overline{e q_{1} e A e q_{1} e}$ such that whenever $p \leq r$ is a nonzero projection, then $\|p b p\|>1-\varepsilon_{3},\|p b p-p\|<\varepsilon_{3}$, and $\|p b-b p\|<$ $\varepsilon_{3}$. By Lemma 1.9] we may require $r \precsim q_{2}$. It follows that, for such $p$, we have $\left\|p e q_{1} e p\right\|>1-3 \varepsilon_{3}$ and $\left\|p e q_{1} e p-p\right\|<\varepsilon_{3}$.

Apply the tracial Rokhlin property with $\{e\} \cup \bigcup_{k=0}^{n-1} \alpha^{k}(F \cup\{u\})$ in place of $F$, with $\varepsilon_{3}$ in place of $\varepsilon$, and with $r$ in place of $x$. Let $f_{0}, f_{1}, \ldots, f_{n-1}$ be the resulting projections, and set $f=\sum_{k=0}^{n-1} f_{k}$.

Since $\left\|\left[f_{k}, e\right]\right\|<\varepsilon_{3}$ for $0 \leq k \leq n-1$, there exist mutually orthogonal projections $g_{0}, g_{1}, \ldots, g_{n-1} \leq e$ such that $\left\|g_{k}-f_{k} e\right\|<\varepsilon_{2}$ for $0 \leq k \leq n-1$. Set $g=\sum_{k=0}^{n-1} g_{k}$. We have

$$
\left\|\alpha\left(g_{k}\right)-g_{k+1}\right\|<2 \varepsilon_{2}+\left\|\alpha\left(f_{k}\right)-f_{k+1}\right\|<3 \varepsilon_{2},
$$

so that $\|\alpha(g)-g\|<3 n \varepsilon_{2}$ and $\left\|\alpha^{k}(g)-g\right\|<3 k n \varepsilon_{2} \leq 3 n^{2} \varepsilon_{2}$. With $b=\frac{1}{n} \sum_{k=0}^{n-1} \alpha^{k}(g)$, we thus get $\|b-g\|<3 n^{2} \varepsilon_{2}$, so the choice of $\varepsilon_{2}$ provides an $\alpha$-invariant projection $h \in e A e$ and a unitary $z \in A$ such that $z g z^{*}=h$ and $\|z-1\|<\frac{1}{8} \varepsilon_{1}$. Set $h_{k}=z g_{k} z^{*}$ for $0 \leq k \leq n-1$. Then $\left\|h_{k}-g_{k}\right\|<\frac{2}{8} \varepsilon_{1}$, so

$$
\left\|h_{k}-f_{k} e\right\|<\frac{2}{8} \varepsilon_{1}+\varepsilon_{2} \leq \frac{3}{8} \varepsilon_{1} .
$$

Since $\alpha(e)=e$, we get

$$
\left\|\alpha\left(h_{k}\right)-h_{k+1}\right\| \leq\left\|h_{k}-f_{k} e\right\|+\left\|h_{k+1}-f_{k+1} e\right\|+\left\|\alpha\left(f_{k}\right)-f_{k+1}\right\|<2\left(\frac{3}{8} \varepsilon_{1}\right)+\varepsilon_{3}<\varepsilon_{1}
$$

for $0 \leq k \leq n-1$,

$$
\left\|\left[h_{j}, \alpha^{k}(u)\right]\right\| \leq 2\left\|h_{k}-f_{k} e\right\|+\left\|\left[f_{j}, \alpha^{k}(u)\right]\right\|<2\left(\frac{3}{8} \varepsilon_{1}\right)+\varepsilon_{3}<\varepsilon_{1}
$$

for $0 \leq k \leq n-1$, and

$$
\left\|\left[h_{j}, \alpha^{k}(a)\right]\right\| \leq 2\left\|h_{k}-f_{k} e\right\|+\left\|\left[f_{j}, \alpha^{k}(a)\right]\right\|+\left\|\left[e, \alpha^{k}(a)\right]\right\|<2\left(\frac{3}{8} \varepsilon_{1}\right)+2 \varepsilon_{3}<\varepsilon_{1}
$$

for $a \in F$ and $0 \leq k \leq n-1$.

We now follow Step 2 of the proof of Proposition 4.1 Set $w_{k}=h_{k} \alpha^{k}(u) h_{k}$ for $0 \leq k \leq n-1$, and set $w=\sum_{k=0}^{n-1} w_{k}$. The same estimates as there give

$$
\left\|w w^{*}-h\right\|<\varepsilon_{1}, \quad\left\|w^{*} w-h\right\|<\varepsilon_{1}, \quad \text { and } \quad\left\|w^{n}-h\right\|<(n-1) \varepsilon_{1} .
$$

As there, $y=\sum_{k=0}^{n-1} \alpha^{k}\left(h_{0} u h_{0}\right) \in h A^{\alpha} h$ satisfies $\|y-w\|<2 n \varepsilon_{1}$, so there is a unitary $v \in h A^{\alpha} h$ such that $v^{n}=h$ and $\|v-w\|<2 n \delta+\varepsilon /(3 n)$. Moreover, also using the same reasoning as there, we have $\left\|v h a h v^{*}-\alpha(h a h)\right\|<\varepsilon / n$ for $a \in F$. Setting $v_{k}=v^{k}$ for $0 \leq k \leq n-1$, and iterating the last estimate as required, we now have Conditions (2), (3), and (4) of Definition 3.2

For Condition (11), we estimate

$$
\left.\|[h, a]\| \leq \sum_{k=0}^{n-1} \| h_{k}, a\right] \|<n \varepsilon_{1} \leq \varepsilon .
$$


For Condition (5), we have $1-e \precsim q_{1}$ by the choice of $e$. Also,

$$
\|(1-f)(e-h)-(e-h)\|=\|f(e-h)\| \leq\|f e-h\| \leq \sum_{k=1}^{n-1}\left\|h_{k}-f_{k} e\right\|<\frac{3}{8} n \varepsilon_{1}<1 .
$$

It follows from Lemma 2.5.2 of [16] and the choice of $f$ that $e-h \precsim 1-f \precsim r \precsim q_{2}$. So $1-h \precsim q_{1}+q_{2} \in \overline{x A x}$, as desired.

It remains only to prove Condition (6). We observed in the previous paragraph that $\|f e-h\|<\frac{3}{8} n \varepsilon_{1}<\frac{1}{2} n \varepsilon_{1}$. The choice of $r$ implies $\left\|r e q_{1} e-r\right\|<2 \varepsilon_{3}$ and $\left\|e q_{1} e r-r\right\|<2 \varepsilon_{3}$. Therefore

$$
\begin{aligned}
\left\|h q_{1} h\right\| & >\left\|f e q_{1} e f\right\|-n \varepsilon_{1}=\left\|q_{1} e f e q_{1}\right\|-n \varepsilon_{1} \geq\left\|r q_{1} e f e q_{1} r\right\|-n \varepsilon_{1} \\
& >\|r f r\|-4 \varepsilon_{3}-n \varepsilon_{1}=\|f r f\|-4 \varepsilon_{3}-n \varepsilon_{1}>1-5 \varepsilon_{3}-n \varepsilon_{1} \geq 1-\frac{1}{2} \varepsilon .
\end{aligned}
$$

So

$$
\begin{aligned}
\|h x h\| & =\left\|x^{1 / 2} h x^{1 / 2}\right\| \geq\left\|q_{1} x^{1 / 2} h x^{1 / 2} q_{1}\right\|=\left\|h x^{1 / 2} q_{1} x^{1 / 2} h\right\| \\
& \geq\left\|h q_{1} x q_{1} h\right\|-2\left\|\left[x^{1 / 2}, q_{1}\right]\right\| \geq\left\|h q_{1} h\right\|-\left\|q_{1} x q_{1}-q_{1}\right\|-2\left\|\left[x^{1 / 2}, q_{1}\right]\right\| \\
& >\left(1-\frac{1}{2} \varepsilon\right)-\delta-2\left(\frac{1}{6} \varepsilon\right) \geq 1-\varepsilon,
\end{aligned}
$$

as desired.

We finish this section by giving one way in which the three main results so far can be combined. This theorem is what is really needed for the classification of higher dimensional noncommutative toruses [23]. We first collect some information from [26].

Proposition 4.7. Let $A$ be a $\mathrm{C}^{*}$-algebra, let $G$ be a compact group, and let $\alpha: G \rightarrow$ Aut $(A)$ be a continuous action of $G$ on $A$. Suppose $C^{*}(G, A, \alpha)$ is simple. Then the fixed point algebra $A^{\alpha}$ is simple, is isomorphic to a full hereditary subalgebra of $C^{*}(G, A, \alpha)$, and is strongly Morita equivalent to $C^{*}(G, A, \alpha)$.

Proof. See the Proposition, Corollary, and proof of the Corollary in [26].

Theorem 4.8. Let $A$ be an infinite dimensional simple separable unital $\mathrm{C}^{*}$-algebra, and let $\alpha \in \operatorname{Aut}(A)$ be a strongly tracially approximately inner automorphism which satisfies $\alpha^{n}=\mathrm{id}_{A}$. Suppose $\alpha$ generates an action of $\mathbb{Z}_{n}$ with the tracial Rokhlin property. Then $A$ has tracial rank zero if and only if the fixed point algebra $A^{\alpha}$ has tracial rank zero.

Proof. Assume that $A$ has tracial rank zero. Theorem 2.6 implies that $C^{*}\left(\mathbb{Z}_{n}, A, \alpha\right)$ has tracial rank zero. Corollary 1.6 implies that $C^{*}\left(\mathbb{Z}_{n}, A, \alpha\right)$ is simple. It therefore follows from Proposition 4.7 that $A^{\alpha}$ is isomorphic to a hereditary subalgebra in $C^{*}\left(\mathbb{Z}_{n}, A, \alpha\right)$. So Theorem 3.12(1) of [14] implies that $A^{\alpha}$ has tracial rank zero.

Now assume that $A^{\alpha}$ has tracial rank zero. By the reverse of the reasoning in the first part of the proof, $C^{*}\left(\mathbb{Z}_{n}, A, \alpha\right)$ has tracial rank zero. It follows from Theorem 4.6 that the action of $\mathbb{Z}_{n}$ on $A$ is tracially approximately representable, and then from Theorem 3.11 that its dual action, which by abuse of notation we call $\widehat{\alpha}$, has the tracial Rokhlin property. So Theorem 2.6 implies that $C^{*}\left(\widehat{\mathbb{Z}_{n}}, C^{*}\left(\mathbb{Z}_{n}, A, \alpha\right), \widehat{\alpha}\right) \cong M_{n} \otimes A$ has tracial rank zero. Then Theorem 3.12(1) of 14 implies that $A$ has tracial rank zero. 


\section{TRACIALLY APPROXIMATELY INNER AUTOMORPHISMS}

In this section, we introduce tracially approximately inner automorphisms, and we prove that they form a group (Theorem [5.8). (We do not know whether the composition of two strongly tracially approximately inner automorphisms is again strongly tracially approximately inner; in fact, we suspect that this is false.) The following definition is an improvement over, and uses a weaker condition than, the definition of tracial approximate innerness given in 22. Again, we do not know, and suspect that it is not true, that the tracially approximately inner automorphisms as defined in 22] form a group.

Definition 5.1. Let $A$ be an infinite dimensional simple separable unital $\mathrm{C}^{*}$ algebra and let $\alpha \in \operatorname{Aut}(A)$. We say that $\alpha$ is tracially approximately inner if for every finite set $F \subset A$, every $\varepsilon>0$, and every positive element $x \in A$ with $\|x\|=1$, there exist $p_{1}, p_{2}, v \in A$ such that:

(1) $p_{1}$ and $p_{2}$ are projections, $v^{*} v=p_{1}$, and $v v^{*}=\alpha\left(p_{2}\right)$.

(2) $\left\|p_{1} a-a p_{1}\right\|<\varepsilon$ and $\left\|p_{2} a-a p_{2}\right\|<\varepsilon$ for all $a \in F$.

(3) $\left\|v p_{1} a p_{1} v^{*}-\alpha\left(p_{2} a p_{2}\right)\right\|<\varepsilon$ for all $a \in F$.

(4) $\frac{1-p_{1}}{x A}$ and $1-p_{2}$ are Murray-von Neumann equivalent to projections in $\overline{x A x}$.

(5) $\left\|p_{1} x p_{1}\right\|>1-\varepsilon$ and $\left\|p_{2} x p_{2}\right\|>1-\varepsilon$.

Lemma 5.2. Let $A$ be an infinite dimensional simple separable unital $\mathrm{C}^{*}$-algebra, and let $\alpha \in \operatorname{Aut}(A)$. If $\alpha$ is approximately inner, then $\alpha$ is tracially approximately inner. If $\alpha$ is tracially approximately inner and $A$ does not have Property (SP), then $\alpha$ is approximately inner.

Proof. The first statement is obvious. For the second, applying the definition with an element $x$ such that $\overline{x A x}$ has no nontrivial projections, one forces $p_{1}=p_{2}=1$, so that $v$ is unitary.

Lemma 5.3. Let $A$ be an infinite dimensional simple separable unital $\mathrm{C}^{*}$-algebra, and let $\alpha \in \operatorname{Aut}(A)$. If $\alpha$ is strongly tracially approximately inner (Definition 4.2), then $\alpha$ is tracially approximately inner.

Proof. This is obvious.

Lemma 5.4. Let $A$ be an infinite dimensional simple separable unital $\mathrm{C}^{*}$-algebra, and let $\alpha \in \operatorname{Aut}(A)$. Suppose that $A$ is finite. Then $\alpha$ is tracially approximately inner if and only if $\alpha$ satisfies the conditions of Definition 5.1 without Part (5).

Proof. Let $\varepsilon>0$, let $F \subset A$ be finite, and let $x \in A$ be positive with $\|x\|=1$. Apply Lemma 1.15] obtaining a projection $q$. Apply the hypotheses, with $\varepsilon$ and $F$ as given, and with $q$ in place of $x$. Then the relations $1-p_{1} \precsim q$ and $1-p_{2} \precsim q$ imply $\left\|p_{1} x p_{1}\right\|>1-\varepsilon$ and $\left\|p_{2} x p_{2}\right\|>1-\varepsilon$.

Lemma 5.5. Let $A$ be an infinite dimensional simple separable unital $\mathrm{C}^{*}$-algebra, and let $\alpha \in \operatorname{Aut}(A)$. Then $\alpha$ is tracially approximately inner if and only if for every finite $F \subset A$, every $\varepsilon>0$, and any three nonzero positive elements $x_{0}, x_{1}, x_{2} \in A$ with $\left\|x_{1}\right\|=\left\|x_{2}\right\|=1$, there are $p_{1}, p_{2}, v \in A$ such that Conditions (1), (2), and (3) of Definition 5.1 hold, and, in addition,

$\left(4^{\prime}\right) \frac{1-p_{1}}{x_{0} A x_{0}}$ and $1-p_{2}$ are Murray-von Neumann equivalent to projections in $x_{0} A x_{0}$. 
$\left(5^{\prime}\right)\left\|p_{1} x_{1} p_{1}\right\|>1-\varepsilon$ and $\left\|p_{2} x_{2} p_{2}\right\|>1-\varepsilon$.

Proof. It is trivial that the condition of the lemma implies tracial approximate innerness. For the reverse direction, if $A$ does not have Property (SP), then $\alpha$ is approximately inner by Lemma 5.2 and the condition of the lemma is immediate. Accordingly, assume that $A$ has Property (SP), let $F \subset A$ be finite, let $\varepsilon>0$, and let $x_{0}, x_{1}, x_{2} \in A$ be nonzero positive elements with $\left\|x_{1}\right\|=\left\|x_{2}\right\|=1$.

Set $\delta=\frac{1}{4} \varepsilon$. Use Lemma 1.14 to choose nonzero projections $q_{1} \in \overline{x_{1} A x_{1}}$ and $q_{2} \in \overline{x_{2} A x_{2}}$ such that, whenever $e \leq q_{j}$ is a nonzero projection, then $\left\|e x_{j} e-e\right\|<\delta$. Use Lemma $\left[1.9\right.$ to find a nonzero projection $e \in \overline{x_{0} A x_{0}}$ and partial isometries $w_{1}, w_{2} \in A$ such that

$$
w_{1}^{*} w_{1}=w_{2}^{*} w_{2}=e, \quad w_{1} w_{1}^{*} \leq q_{1}, \quad \text { and } \quad w_{2} w_{2}^{*} \leq q_{2} .
$$

Apply Definition 5.1 with $F \cup\left\{w_{1}, w_{1}^{*}, w_{2}, w_{2}^{*}\right\}$ in place of $F$, with $\delta$ in place of $\varepsilon$, and with $e$ in place of $x$, obtaining $p_{1}, p_{2}$, and $v$. It is immediate that Conditions (11), (21), and (3) of Definition 5.1] and Condition $\left(4^{\prime}\right)$ of the present lemma hold. It remains to prove Condition $\left(5^{\prime}\right)$.

Using

$$
\left\|\left(w_{1} w_{1}^{*}\right) x_{1}\left(w_{1} w_{1}^{*}\right)-w_{1} w_{1}^{*}\right\|<\delta, \quad w_{1}^{*} w_{1} w_{1}^{*}=w_{1}^{*}, \quad \text { and } \quad\left(w_{1}^{*} w_{1}\right)^{2}=e,
$$

we get $\left\|w_{1}^{*} x_{1} w_{1}-e\right\|<\delta$. Therefore

$$
\begin{aligned}
\left\|p_{1} x_{1} p_{1}\right\| & \geq\left\|w_{1}^{*} p_{1} x_{1} p_{1} w_{1}\right\| \geq\left\|p_{1} w_{1}^{*} x_{1} w_{1} p_{1}\right\|-\left\|\left[p_{1}, w_{1}\right]\right\|-\left\|\left[p_{1}, w_{1}^{*}\right]\right\| \\
& >\left\|p_{1} e p_{1}\right\|-\delta-\left\|\left[p_{1}, w_{1}\right]\right\|-\left\|\left[p_{1}, w_{1}^{*}\right]\right\|>1-4 \delta=1-\varepsilon .
\end{aligned}
$$

The same reasoning gives $\left\|p_{2} x_{2} p_{2}\right\|>1-\varepsilon$.

For the proof of the next result, it is convenient to observe that the relations in the definition of tracial approximate innerness need only hold approximately.

Lemma 5.6. Let $A$ be an infinite dimensional simple separable unital $\mathrm{C}^{*}$-algebra, and let $\alpha \in \operatorname{Aut}(A)$. Suppose that for every $\varepsilon>0$, every finite $F \subset A$, and any three nonzero positive elements $x_{0}, x_{1}, x_{2} \in A$ with $\left\|x_{1}\right\|=\left\|x_{2}\right\|=1$, there are $e_{1}, e_{2}, y \in A$ such that:

(1) $\left\|e_{1}\right\|,\left\|e_{2}\right\|,\|y\| \leq 1$.

(2) $\left\|e_{1}^{2}-e_{1}\right\|,\left\|e_{1}^{*}-e_{1}\right\|,\left\|e_{2}^{2}-e_{2}\right\|,\left\|e_{2}^{*}-e_{2}\right\|,\left\|y^{*} y-e_{1}\right\|$, and $\left\|y y^{*}-\alpha\left(e_{2}\right)\right\|$ are all less than $\varepsilon$.

(3) $\left\|e_{1} a-a e_{1}\right\|<\varepsilon$ and $\left\|e_{2} a-a e_{2}\right\|<\varepsilon$ for all $a \in F$.

(4) $\left\|y e_{1} a e_{1} y^{*}-\alpha\left(e_{2} a e_{2}\right)\right\|<\varepsilon$ for all $a \in F$.

(5) $\frac{1}{2} \notin \operatorname{sp}\left(e_{1}^{*} e_{1}\right) \cup \operatorname{sp}\left(e_{2}^{*} e_{2}\right)$, and $1-\chi_{[1 / 2, \infty)}\left(e_{1}^{*} e_{1}\right)$ and $1-\chi_{[1 / 2, \infty)}\left(e_{2}^{*} e_{2}\right)$ are Murray-von Neumann equivalent to projections in $\overline{x_{0} A x_{0}}$.

(6) $\left\|e_{1} x_{1} e_{1}\right\|>1-\varepsilon$ and $\left\|e_{2} x_{2} e_{2}\right\|>1-\varepsilon$.

Then $\alpha$ is tracially approximately inner.

Proof. Let $F, \varepsilon, x_{0}, x_{1}, x_{2}$ be as in the hypotheses of Lemma 5.5. Without loss of generality $\|a\| \leq 1$ for all $a \in F$. Choose $\varepsilon_{0}>0$ with $\varepsilon_{0} \leq \frac{1}{7} \varepsilon$ and so small that whenever $p_{1}, p_{2} \in A$ are projections, and $y \in A$ satisfies $\left\|y^{*} y-p_{1}\right\|<2 \varepsilon_{0}$ and $\left\|y y^{*}-p_{2}\right\|<2 \varepsilon_{0}$, then there is a partial isometry $v \in A$ such that

$$
\|v-y\|<\frac{1}{7} \varepsilon, \quad v^{*} v=p_{1}, \quad \text { and } \quad v v^{*}=p_{2} .
$$

Choose $\delta>0$ with $\delta \leq \varepsilon_{0}$ and also so small that whenever $e \in A$ satisfies $\left\|e^{2}-e\right\|<\delta$ and $\left\|e^{*}-e\right\|<\delta$, then $p=\chi_{[1 / 2, \infty)}\left(e^{*} e\right)$ is defined and satisfies $\|e-p\|<\varepsilon_{0}$. 
Apply the conditions of the lemma with $\delta$ in place of $\varepsilon$, obtaining $e_{1}, e_{2}$, and $y$. Set $p_{1}=\chi_{[1 / 2, \infty)}\left(e_{1}^{*} e_{1}\right)$ and $p_{2}=\chi_{[1 / 2, \infty)}\left(e_{2}^{*} e_{2}\right)$, giving $\left\|p_{1}-e_{1}\right\|<\varepsilon_{0}$ and $\left\|p_{2}-e_{2}\right\|<$ $\varepsilon_{0}$. Then $\left\|y^{*} y-p_{1}\right\|<\delta+\varepsilon_{0} \leq 2 \varepsilon_{0}$, and similarly $\left\|y y^{*}-\alpha\left(p_{2}\right)\right\|<2 \varepsilon_{0}$. Therefore there is a partial isometry $v \in A$ such that

$$
\|v-y\|<\frac{1}{7} \varepsilon, \quad v^{*} v=p_{1}, \quad \text { and } \quad v v^{*}=\alpha\left(p_{2}\right) .
$$

Since also

$$
\left\|p_{1}-e_{1}\right\|<\frac{1}{7} \varepsilon, \quad\left\|p_{2}-e_{2}\right\|<\frac{1}{7} \varepsilon, \quad\left\|e_{1} x e_{1}\right\|>1-\frac{1}{7} \varepsilon, \quad \text { and } \quad\left\|e_{2} x e_{2}\right\|>1-\frac{1}{7} \varepsilon,
$$

we easily obtain the conditions of Lemma [5.5

Proposition 5.7. Let $A$ be an infinite dimensional simple separable unital $\mathrm{C}^{*}$ algebra, and let $\alpha, \beta \in \operatorname{Aut}(A)$ be tracially approximately inner. Then $\alpha \circ \beta$ is tracially approximately inner.

Proof. If $A$ does not have Property (SP), then $\alpha$ and $\beta$ are approximately inner by Lemma [5.2 so $\alpha \circ \beta$ is approximately inner. Thus, we assume that $A$ has Property (SP).

We verify the hypotheses of Lemma [5.6 Let $F \subset A$ be finite, let $\varepsilon>0$, and let $x_{0}, x_{1}, x_{2} \in A$ be nonzero positive elements with $\left\|x_{1}\right\|=\left\|x_{2}\right\|=1$. Without loss of generality $\|a\| \leq 1$ for all $a \in F$.

Choose a nonzero projection $r \in \overline{x_{0} A x_{0}}$, and use Lemma 1.10 to choose orthogonal nonzero projections $r_{1}^{(0)}, r_{2} \leq r$. Then use Lemma 1.9 to choose a nonzero projection $r_{1} \leq r_{1}^{(0)}$ such that $r_{1} \precsim \beta\left(r_{1}^{(0)}\right)$.

Choose $\delta>0$ with $\delta \leq \min \left(\frac{\widetilde{1}}{6} \varepsilon, \frac{1}{40}\right)$, and also so small that whenever $B$ is a $\mathrm{C}^{*}$-algebra and $p, q \in B$ are projections such that $\|[p, q]\|<\delta$, then there exists a projection $g \in B$ which commutes with $p$ and satisfies $\|g-q\|<\frac{1}{4}$. Apply the condition of Lemma 5.5 to $\beta$, with $F$ as given, with $\delta$ in place of $\varepsilon$, with $r_{1}$ in place of $x_{0}$, and with $x_{1}$ and $x_{2}$ as given. Let $q_{1}, q_{2}, w \in A$ be the resulting projections and partial isometry.

Set

$$
E=F \cup \beta(F) \cup\left\{\beta\left(q_{2} a q_{2}\right): a \in F\right\} \cup\left\{w, w^{*}, q_{1}, q_{2}, \beta\left(q_{2}\right)\right\} .
$$

Set $\lambda_{1}=\left\|q_{1} x_{1} q_{1}\right\|$ and $\lambda_{2}=\left\|q_{2} x_{2} q_{2}\right\|$. Apply the condition of Lemma 5.5 to $\alpha$, with $E$ in place of $F$, with $\delta$ in place of $\varepsilon$, with $r_{2}$ in place of $x_{0}$, with $\lambda_{1}^{-1} q_{1} x_{1} q_{1}$ in place of $x_{1}$, and with $\lambda_{2}^{-1} \beta\left(q_{2} x_{2} q_{2}\right)$ in place of $x_{2}$. Let $p_{1}, p_{2}, v \in A$ be the resulting projections and partial isometry.

Set

$$
e_{1}=p_{1} q_{1}, \quad e_{2}=\beta^{-1}\left(p_{2}\right) q_{2}, \quad \text { and } \quad y=v w .
$$

Condition (11) of Lemma [5.6 is obvious. We have $\left\|e_{1}^{2}-e_{1}\right\| \leq\left\|\left[p_{1}, q_{1}\right]\right\|<\delta \leq \varepsilon$, and the same estimate applies to $\left\|e_{1}^{*}-e_{1}\right\|$. Also,

$$
\left\|e_{2}^{2}-e_{2}\right\| \leq\left\|\left[\beta^{-1}\left(p_{2}\right), q_{2}\right]\right\|=\left\|\left[p_{2}, \beta\left(q_{2}\right)\right]\right\|<\delta \leq \varepsilon,
$$

and again the same estimate applies to $\left\|e_{2}^{*}-e_{2}\right\|$. Next, since $v^{*} v=p_{1}$ and $w^{*} w=$ $q_{1}$, we get

$$
\left\|y^{*} y-e_{1}\right\|=\left\|w^{*} p_{1} w-p_{1} q_{1}\right\| \leq\left\|\left[w^{*}, p_{1}\right]\right\|<\delta \leq \varepsilon .
$$

Furthermore, by the choice of $p_{1}, p_{2}$, and $v$, and by the definition of $e_{2}$, we have

$$
\left\|y y^{*}-(\alpha \circ \beta)\left(e_{2} e_{2}^{*}\right)\right\|=\left\|v p_{1} \beta\left(q_{2}\right) p_{1} v^{*}-\alpha\left(p_{2} \beta\left(q_{2}\right) p_{2}\right)\right\|<\delta .
$$

Since $\left\|e_{2} e_{2}^{*}-e_{2}\right\| \leq\left\|e_{2}^{*}-e_{2}\right\|+\left\|e_{2}^{2}-e_{2}\right\|<2 \delta$, we get $\left\|y y^{*}-(\alpha \circ \beta)\left(e_{2}\right)\right\|<3 \delta \leq \varepsilon$. This verifies (2) of Lemma [5.6 
For (31), for $a \in F$ we estimate

$$
\left\|\left[p_{1} q_{1}, a\right]\right\| \leq\left\|p_{1}\right\| \cdot\left\|\left[q_{1}, a\right]\right\|+\left\|\left[p_{1}, a\right]\right\| \cdot\left\|q_{1}\right\|<2 \delta \leq \varepsilon
$$

and

$$
\left\|\left[\beta^{-1}\left(p_{2}\right) q_{2}, a\right]\right\| \leq\left\|\beta^{-1}\left(p_{2}\right)\right\| \cdot\left\|\left[q_{2}, a\right]\right\|+\left\|\left[p_{2}, \beta(a)\right]\right\| \cdot\left\|q_{2}\right\|<2 \delta \leq \varepsilon .
$$

We verify (4). We have

$$
\begin{aligned}
& \left\|y e_{1} a e_{1} y^{*}-(\alpha \circ \beta)\left(e_{2} a e_{2}\right)\right\| \\
& \qquad\left\|v w p_{1} q_{1} a p_{1} q_{1} w^{*} v^{*}-(\alpha \circ \beta)\left(\beta^{-1}\left(p_{2}\right) q_{2} a \beta^{-1}\left(p_{2}\right) q_{2}\right)\right\| \\
& \leq 2\left\|\left[w, p_{1}\right]\right\|+\left\|\left[p_{1}, q_{1}\right]\right\|+\left\|\left[\beta^{-1}\left(p_{2}\right), q_{2}\right]\right\| \\
& \quad+\left\|v p_{1} w q_{1} a q_{1} w^{*} p_{1} v^{*}-\alpha\left(p_{2} \beta\left(q_{2} a q_{2}\right) p_{2}\right)\right\| \\
& \leq 2\left\|\left[w, p_{1}\right]\right\|+\left\|\left[p_{1}, q_{1}\right]\right\|+\left\|\left[p_{2}, \beta\left(q_{2}\right)\right]\right\|+\left\|w q_{1} a q_{1} w^{*}-\beta\left(q_{2} a q_{2}\right)\right\| \\
& \quad+\left\|v p_{1} \beta\left(q_{2} a q_{2}\right) p_{1} v^{*}-\alpha\left(p_{2} \beta\left(q_{2} a q_{2}\right) p_{2}\right)\right\| \\
& <6 \delta \leq \varepsilon,
\end{aligned}
$$

as desired.

Next, we prove (5]) of Lemma 5.6. We have

$$
\left\|\left(e_{1}^{*} e_{1}\right)^{2}-e_{1}^{*} e_{1}\right\| \leq 2\left\|e_{1}^{*}-e_{1}\right\|+2\left\|e_{1}^{2}-e_{1}\right\|<4 \delta \leq \frac{1}{10},
$$

so that $\frac{1}{2} \notin \operatorname{sp}\left(e_{1}^{*} e_{1}\right)$, the projection $f_{1}=\chi_{[1 / 2, \infty)}\left(e_{1}^{*} e_{1}\right)$ is defined, and it satisfies $\left\|e_{1}^{*} e_{1}-f_{1}\right\|<\frac{1}{5}$. So

$$
\left\|f_{1}-e_{1}\right\| \leq\left\|f_{1}-e_{1}^{*} e_{1}\right\|+\left\|e_{1}^{*}-e_{1}\right\|+\left\|e_{1}^{2}-e_{1}\right\|<\frac{1}{5}+\delta+\delta \leq \frac{1}{4} .
$$

Moreover, by the choice of $\delta$ and because $\left\|\left[p_{1}, q_{1}\right]\right\|<\delta$, there is a projection $g_{1} \in A$ which commutes with $p_{1}$ and such that $\left\|g_{1}-q_{1}\right\|<\frac{1}{4}$. It follows that

$$
\left\|(1-f)-\left[\left(1-p_{1}\right)+p_{1}\left(1-g_{1}\right)\right]\right\|=\left\|p_{1} g_{1}-f_{1}\right\| \leq\left\|g_{1}-q_{1}\right\|+\left\|e_{1}-f_{1}\right\|<\frac{1}{2} .
$$

Therefore

$$
\begin{aligned}
1-f_{1} & \sim\left(1-p_{1}\right)+p_{1}\left(1-g_{1}\right) \\
& \precsim\left(1-p_{1}\right) \oplus\left(1-g_{1}\right) \sim\left(1-p_{1}\right) \oplus\left(1-q_{1}\right) \precsim r_{2}+r_{1} \leq r \in \overline{x_{0} A x_{0}} .
\end{aligned}
$$

Applying the same argument with $\beta^{-1}\left(p_{2}\right)$ in place of $p_{1}$ and with $q_{2}$ in place of $q_{1}$, it follows that the projection $f_{2}=\chi_{[1 / 2, \infty)}\left(e_{2}^{*} e_{2}\right)$ is defined, it satisfies $\left\|e_{2}^{*} e_{2}-f_{2}\right\|<\frac{1}{5}$, and

$$
1-f_{2} \precsim r_{2} \oplus \beta^{-1}\left(r_{1}\right) \precsim r_{2}+r_{1}^{(0)} \leq r \in \overline{x_{0} A x_{0}} .
$$

It remains only to prove (6). Since $\frac{1}{2}<\lambda_{1} \leq 1$, we have

$$
1 \leq \lambda_{1}^{-1}<1+2\left(1-\lambda_{1}\right)<1+2 \delta \text {. }
$$

Therefore

$$
\left\|\lambda_{1}^{-1} p_{1} q_{1} x_{1} q_{1} p_{1}-p_{1} q_{1} x_{1} q_{1} p_{1}\right\|<2 \delta
$$

so that, using $\left\|\left[p_{1}, q_{1}\right]\right\|<\delta$ at the first step,

$$
\left\|e_{1} x_{1} e_{1}\right\|>\left\|p_{1} q_{1} x_{1} q_{1} p_{1}\right\|-\delta>\left\|\lambda_{1}^{-1} p_{1} q_{1} x_{1} q_{1} p_{1}\right\|-3 \delta>1-4 \delta \geq 1-\varepsilon .
$$

Similar reasoning gives

$$
\left\|e_{2} x_{2} e_{2}\right\|>\left\|\beta^{-1}\left(p_{2}\right) q_{2} x_{2} q_{2} \beta^{-1}\left(p_{2}\right)\right\|-\delta>\left\|\lambda_{2}^{-1} p_{2} \beta\left(q_{2} x_{2} q_{2}\right) p_{2}\right\|-3 \delta>1-\varepsilon .
$$

This completes the proof. 
Theorem 5.8. Let $A$ be an infinite dimensional simple separable unital $\mathrm{C}^{*}$-algebra. Then the set of tracially approximately inner automorphisms of $A$ is a group.

Proof. Obviously $\mathrm{id}_{A}$ is tracially approximately inner. In view of Proposition [5.7 we need only prove that if $\alpha$ is tracially approximately inner, then so is $\alpha^{-1}$. We use the condition given in Lemma 5.5

Let $F \subset A$ be finite, let $\varepsilon>0$, and let $x_{0}, x_{1}, x_{2} \in A$ be nonzero positive elements with $\left\|x_{1}\right\|=\left\|x_{2}\right\|=1$. Apply the condition of Lemma [5.5 with $F, \varepsilon$, and $x_{0}$ as given, with $x_{2}$ in place of $x_{1}$, and with $x_{1}$ in place of $x_{2}$. Let $q_{1}, q_{2}, w \in A$ be the resulting projections and partial isometry. Then set $p_{1}=q_{2}, p_{2}=q_{1}$, and $v=\alpha^{-1}\left(w^{*}\right)$. These satisfy the conditions in Lemma 5.5

\section{Properties of tRACIALly APPROXIMATELY INNER AUTOMORPHiSMS}

In this section, we prove that a tracially approximately inner automorphism is necessarily trivial on the tracial state space and on $K_{0} \bmod$ infinitesimals. On an infinite dimensional simple separable unital $\mathrm{C}^{*}$-algebra with tracial rank zero, an automorphism which is trivial on $K_{0}$ mod infinitesimals is necessarily tracially approximately inner; this is the analog of the fact that an automorphism of an AF algebra which is trivial on $K_{0}$ is necessarily approximately inner. We also prove that if a tracially approximately inner automorphism of an infinite dimensional simple separable unital $\mathrm{C}^{*}$-algebra with tracial rank zero has finite order, then it is strongly tracially approximately inner in the sense of Definition 4.2 Thus, the results of Section 4 apply to such automorphisms. (This does not help with our main application of those results [23], because we use them as part of the proof that the algebra involved has tracial rank zero.)

Proposition 6.1. Let $A$ be an infinite dimensional simple separable unital $\mathrm{C}^{*}$ algebra, and let $\alpha \in \operatorname{Aut}(A)$ be tracially approximately inner. Then $\tau \circ \alpha=\tau$ for every tracial state $\tau$ on $A$.

Proof. If $\alpha$ is approximately inner, the result is immediate. We may therefore assume that $A$ has Property (SP). Let $\tau$ be a tracial state on $A$, let $\varepsilon>0$, and let $a \in A$. We prove that $|\tau(\alpha(a))-\tau(a)|<\varepsilon$. Without loss of generality $\|a\|=1$.

Choose $n$ such that $\frac{1}{n}<\frac{1}{3} \varepsilon$. By page 61 of [1], there exists a selfadjoint $b \in A$ such that $\operatorname{sp}(a)=[0,1]$. Choose continuous functions

$$
f_{1}, f_{2}, \ldots, f_{n}, g_{1}, g_{2}, \ldots, g_{n}:[0,1] \rightarrow[0,1]
$$

such that $\left\|g_{k}\right\|=1$ and $f_{k} g_{k}=g_{k}$ for $1 \leq k \leq n$, and such that the supports of $f_{1}, f_{2}, \ldots, f_{n}$ are disjoint. Then $\sum_{k=1}^{n} \tau\left(f_{k}(b)\right) \leq 1$, so there exists $k$ such that $\tau\left(f_{k}(b)\right) \leq \frac{1}{n}$. Choose a nonzero projection $q_{0} \in \overline{g_{k}(b) A g_{k}(b)}$, and use Lemma 1.9 to find a nonzero projection $q \leq q_{0}$ such that $q \precsim \alpha^{-1}\left(q_{0}\right)$. Since $f_{k}(b) q_{0}=q_{0}$, we have $\tau\left(q_{0}\right) \leq \tau\left(f_{k}(b)\right) \leq \frac{1}{n}$, so that $\tau(q) \leq \frac{1}{n}$ and $\tau(\alpha(q)) \leq \frac{1}{n}$.

Apply Definition [5.1 with $F=\{a\}$, with $\frac{1}{3} \varepsilon$ in place of $\varepsilon$, and with $x=q$, obtaining $p_{1}, p_{2}$, and $v$. Since $\|a\| \leq 1$, we have

$\left|\tau(a)-\tau\left(p_{1} a p_{1}\right)\right| \leq \tau\left(1-p_{1}\right) \leq \frac{1}{n} \quad$ and $\quad\left|\tau(\alpha(a))-\tau\left(\alpha\left(p_{2} a p_{2}\right)\right)\right| \leq \tau\left(\alpha\left(1-p_{2}\right)\right) \leq \frac{1}{n}$.

Also,

$$
\tau\left(v p_{1} a p_{1} v^{*}\right)=\tau\left(v^{*} v p_{1} a p_{1}\right)=\tau\left(p_{1} a p_{1}\right)
$$


$\mathrm{SO}$

$$
\begin{aligned}
|\tau(\alpha(a))-\tau(a)| \leq & \left|\tau(\alpha(a))-\tau\left(\alpha\left(p_{2} a p_{2}\right)\right)\right| \\
& \quad+\left\|\alpha\left(p_{2} a p_{2}\right)-v p_{1} a p_{1} v^{*}\right\|+\left|\tau\left(p_{1} a p_{1}\right)-\tau(a)\right| \\
< & \frac{1}{n}+\frac{1}{n}+\frac{1}{3} \varepsilon<\varepsilon .
\end{aligned}
$$

This completes the proof.

Recall that an element $\eta$ of a partially ordered group $G$ with order unit $u \in$ $G_{+} \backslash\{0\}$ is infinitesimal if $-m u \leq n \eta \leq m u$ for all $m, n \in \mathbb{N}$ with $m>0$. See Definition 1.10 of $[8$, where this definition is given for simple dimension groups. Clearly we need only consider $m=1$. By Proposition 4.7 of [9], an equivalent condition is that all states on $(G, u)$ vanish on $\eta$.

Proposition 6.2. Let $A$ be an infinite dimensional simple separable unital $\mathrm{C}^{*}$ algebra, and let $\alpha \in \operatorname{Aut}(A)$ be tracially approximately inner. Then $\alpha_{*}(\eta)-\eta$ is infinitesimal for every $\eta \in K_{0}(A)$.

Proof. If $A$ does not have Property (SP), then $\alpha$ is approximately inner by Lemma 5.2 so $\alpha_{*}(\eta)-\eta=0$ for every $\eta \in K_{0}(A)$. So assume that $A$ has Property (SP).

We prove that for every $\eta \in K_{0}(A)$ we have $-\left[1_{A}\right] \leq \alpha_{*}(\eta)-\eta \leq\left[1_{A}\right]$. This implies the result, because replacing $\eta$ by $n \eta$ gives $-\left[1_{A}\right] \leq n\left[\alpha_{*}(\eta)-\eta\right] \leq\left[1_{A}\right]$.

Accordingly, let $\eta \in K_{0}(A)$, and choose $n \in \mathbb{N}$ and projections $p, r \in M_{n}(A)$ such that $\eta=[q]-[r]$. Let $\chi: \mathbb{R} \backslash\left\{\frac{1}{2}\right\} \rightarrow \mathbb{R}$ be the characteristic function of $\left(\frac{1}{2}, \infty\right)$. Choose $\varepsilon>0$ so small that $n^{2} \varepsilon<\frac{1}{6}$, and also so small that whenever $C$ is a $C^{*}$-algebra and $f, p \in C$ are projections such that $\|f p-p f\|<n^{2} \varepsilon$, then $\frac{1}{2}$ is not in the spectrum of either $f p f$ or $(1-f) p(1-f)$, and moreover the projections $p_{0}=\chi(f p f)$ and $p_{1}=\chi((1-f) p(1-f))$ satisfy $\left\|p_{0}+p_{1}-p\right\|<\frac{1}{6}$. Use Lemma 1.10 to choose $2 n$ nonzero mutually orthogonal projections $g_{1}, g_{2}, \ldots, g_{2 n} \in A$, and use Lemma 1.9 to choose a nonzero projection $h \leq g_{1}$ such that $\alpha(h) \leq g_{1}$.

Apply Definition 5.1 with $F=\left\{q_{j, k}, r_{j, k}: 1 \leq j, k \leq n\right\}$, the set of all matrix entries of $q$ and $r$, with $\varepsilon$ as just chosen, and with $x=h$. Let $p_{1}, p_{2}, v \in e A e$ be the resulting projections and partial isometry.

We have

$$
\left\|\left(1 \otimes p_{1}\right) q-q\left(1 \otimes p_{1}\right)\right\| \leq \sum_{j, k=1}^{n}\left\|p_{1} q_{j, k}-q_{j, k} p_{1}\right\|<n^{2} \varepsilon .
$$

By the choice of $\varepsilon$ the projections

$$
q_{1}=\chi\left(\left(1 \otimes p_{1}\right) q\left(1 \otimes p_{1}\right)\right) \in M_{n}\left(p_{1} A p_{1}\right)
$$

and

$$
e_{1}=\chi\left(\left(1-1 \otimes p_{1}\right) q\left(1-1 \otimes p_{1}\right)\right) \in M_{n}\left(\left(1-p_{1}\right) A\left(1-p_{1}\right)\right)
$$

are defined and satisfy $\left\|q_{1}+e_{1}-q\right\|<\frac{1}{6}$. Similarly,

$$
q_{2}=\chi\left(\left(1 \otimes p_{2}\right) q\left(1 \otimes p_{2}\right)\right) \in M_{n}\left(p_{2} A p_{2}\right)
$$

and

$$
e_{2}=\chi\left(\left(1-1 \otimes p_{2}\right) q\left(1-1 \otimes p_{2}\right)\right) \in M_{n}\left(\left(1-p_{2}\right) A\left(1-p_{2}\right)\right)
$$

are defined and satisfy $\left\|q_{2}+e_{2}-q\right\|<\frac{1}{6}$. Note in particular that

$$
[q]=\left[q_{1}\right]+\left[e_{1}\right]=\left[q_{2}\right]+\left[e_{2}\right]
$$

in $K_{0}(A)$. 
We now claim that $\left[q_{2}\right]=\left[\alpha\left(q_{1}\right)\right]$ in $K_{0}(A)$. First,

$$
\left\|\left(1 \otimes p_{1}\right) q\left(1 \otimes p_{1}\right)-q_{1}\right\|=\left\|\left(1 \otimes p_{1}\right)\left[q-\left(q_{1}+e_{1}\right)\right]\left(1 \otimes p_{1}\right)\right\|<\frac{1}{6} .
$$

Similarly,

$$
\left\|\left(1 \otimes p_{2}\right) q\left(1 \otimes p_{2}\right)-q_{2}\right\|<\frac{1}{6}
$$

Finally,

$$
\begin{array}{r}
\left\|(1 \otimes v)\left(1 \otimes p_{1}\right) q\left(1 \otimes p_{1}\right)(1 \otimes v)^{*}-(\mathrm{id} \otimes \alpha)\left(\left(1 \otimes p_{2}\right) q\left(1 \otimes p_{2}\right)\right)\right\| \\
\leq \sum_{j, k=1}^{n}\left\|v p_{1} q_{j, k} p_{1} v^{*}-\alpha\left(p_{2} q_{j, k} p_{2}\right)\right\|<n^{2} \varepsilon .
\end{array}
$$

Putting these estimates together gives

$$
\left\|(1 \otimes v) q_{2}(1 \otimes v)^{*}-\alpha\left(q_{1}\right)\right\|<\frac{1}{6}+n^{2} \varepsilon+\frac{1}{6}<\frac{1}{2} .
$$

The claim follows.

Repeating the argument of the last two paragraphs with $r$ in place of $q$, we find projections

$$
r_{1} \in M_{n}\left(p_{1} A p_{1}\right), \quad f_{1} \in M_{n}\left(\left(1-p_{1}\right) A\left(1-p_{1}\right)\right)
$$

and

$$
r_{2} \in M_{n}\left(p_{2} A p_{2}\right), \quad f_{2} \in M_{n}\left(\left(1-p_{2}\right) A\left(1-p_{2}\right)\right)
$$

such that

$$
[r]=\left[r_{1}\right]+\left[f_{1}\right]=\left[r_{2}\right]+\left[f_{2}\right] \text { and }\left[r_{2}\right]=\left[\alpha\left(r_{1}\right)\right]
$$

in $K_{0}(A)$.

We have

$$
\alpha_{*}(\eta)-\eta=\alpha_{*}([q])-[q]-\alpha_{*}([r])+[r]=\alpha_{*}\left(\left[e_{2}\right]\right)-\left[e_{1}\right]-\alpha_{*}\left(\left[f_{2}\right]\right)+\left[f_{1}\right] .
$$

Since

$$
e_{1} \in M_{n}\left(\left(1-p_{1}\right) A\left(1-p_{1}\right)\right) \text { and } 1-p_{1} \precsim h \leq g_{1} \text {, }
$$

and since

$$
f_{2} \in M_{n}\left(\left(1-p_{2}\right) A\left(1-p_{2}\right)\right) \quad \text { and } \quad 1-\alpha\left(p_{2}\right) \precsim \alpha(h) \precsim g_{1},
$$

in $K_{0}(A)$ we have

$$
\left[e_{1}\right]+\alpha_{*}\left(\left[f_{2}\right]\right) \leq n\left[1-p_{1}\right]+n\left[1-\alpha\left(p_{2}\right)\right] \leq n[h]+n[\alpha(h)] \leq 2 n\left[g_{1}\right] \leq\left[1_{A}\right] .
$$

Similarly, $\alpha_{*}\left(\left[e_{2}\right]\right)+\left[f_{1}\right] \leq\left[1_{A}\right]$. Therefore

$$
-\left[1_{A}\right] \leq-\left[e_{1}\right]-\alpha_{*}\left(\left[f_{2}\right]\right) \leq \alpha_{*}(\eta)-\eta \leq \alpha_{*}\left(\left[e_{2}\right]\right)+\left[f_{1}\right] \leq\left[1_{A}\right] .
$$

This completes the proof.

We now prove that if $A$ has tracial rank zero, then there is a converse to Proposition 6.2 We give a preliminary lemma.

Lemma 6.3. Let $A$ be a simple unital $\mathrm{C}^{*}$-algebra with Property (SP), with stable rank one, and such that the order on projections over $A$ is determined by traces (Definition 2.4). Let $p, q \in A$ be projections such that $[p]-[q]$ is infinitesimal in $K_{0}(A)$. Then for every nonzero positive element $x \in A$ there exist projections $p_{0} \leq p$ and $q_{0} \leq q$ such that $p_{0} \sim q_{0}$ and such that $p-p_{0}$ and $q-q_{0}$ are Murray-von Neumann equivalent to projections in $\overline{x A x}$. 
Proof. For every tracial state $\tau$ on $A$, we have $-\tau_{*}\left(\left[1_{A}\right]\right) \leq n \tau_{*}([p]-[q]) \leq \tau_{*}\left(\left[1_{A}\right]\right)$ for all $n \in \mathbb{N}$. Therefore $\tau(p)=\tau(q)$. Since $A$ is simple, if $p \in\{0,1\}$ then $q=p$, and there is nothing to prove. Accordingly, we may assume $p \notin\{0,1\}$.

Lemma 1.10 gives orthogonal nonzero projections $r, s \in \overline{x A x}$. Use Lemma 1.9 to choose nonzero projections $e \leq p$ and $e_{0}$ such that $e \sim e_{0} \leq r$, and $f \leq 1-p$ and $f_{0}$ such that $f \sim f_{0} \leq s$. Set $p_{0}=p-e$. For every tracial state $\tau$ on $A$, we have $\tau(q)-\tau\left(p_{0}\right)=\tau(e)>0$. Since the order on projections over $A$ is determined by traces, there exists a projection $q_{0} \leq q$ such that $q_{0} \sim p_{0}$. Similarly (also using stable rank one), there exists a projection $q_{1} \geq q$ such that $p+f \sim q_{1}$.

Clearly

$$
p-p_{0}=e \precsim r \leq r+s \in \overline{x A x} .
$$

Also,

$$
q-q_{0} \leq q_{1}-q_{0} \sim e+f \precsim r+s \in \overline{x A x} .
$$

This completes the proof.

Theorem 6.4. Let $A$ be an infinite dimensional simple separable unital $\mathrm{C}^{*}$-algebra with tracial rank zero. Let $\alpha \in \operatorname{Aut}(A)$ be an automorphism such that $\alpha_{*}(\eta)-\eta$ is infinitesimal for every $\eta \in K_{0}(A)$. Then $\alpha$ is tracially approximately inner.

Proof. Let $F \subset A$ be finite, let $\varepsilon>0$, and let $x \in A$ be a positive element with $\|x\|=1$. Set $\varepsilon_{0}=\frac{1}{6} \varepsilon$. Choose a nonzero projection $r \in \overline{x A x}$. By Lemma 1.9] there exists a nonzero projection $r \leq r_{0}$ such that $r-r_{0} \neq 0$. Use Proposition 2.3 to choose a projection $p \in A$ and a finite dimensional unital subalgebra $E \subset p A p$ such that:

(1) $\|p a-a p\|<\varepsilon_{0}$ for all $a \in F$.

(2) For every $a \in F$ there exists $b \in E$ such that $\|p a p-b\|<\varepsilon_{0}$.

(3) $1-p \precsim r_{0}$.

Write $E=\bigoplus_{l=1}^{m} E_{l}$ with $E_{l} \cong M_{n(l)}$ for $1 \leq l \leq m$. Let $\left(e_{j, k}^{(l)}\right)_{1 \leq j, k \leq n(l)}$ be a system of matrix units for $E_{l}$.

Choose (Lemma 1.10) orthogonal nonzero equivalent projections $r_{l, j} \leq r-r_{0}$ for $1 \leq l \leq m$ and $1 \leq j \leq n(l)$. By Lemma 1.9 there exist equivalent nonzero projections $s_{l, j} \leq r_{l, j}$ such that $\alpha^{-1}\left(s_{l, j}\right) \precsim r_{l, j}$. For $1 \leq l \leq m$ use Lemma 6.3 and Theorem 2.5 to find projections $f_{l} \leq e_{1,1}^{(l)}$ and $g_{l} \leq \alpha\left(e_{1,1}^{(l)}\right)$, and $v_{l} \in A$, such that

$$
v_{l}^{*} v_{l}=f_{l}, \quad v_{l} v_{l}^{*}=g_{l}, \quad e_{1,1}^{(l)}-f_{l} \precsim s_{l, 1}, \quad \text { and } \quad \alpha\left(e_{1,1}^{(l)}\right)-g_{l} \precsim s_{l, 1} .
$$

Define

$f=\sum_{l=1}^{m} \sum_{j=1}^{n(l)} e_{j, 1}^{(l)} f_{l} e_{1, j}^{(l)}, \quad g=\sum_{l=1}^{m} \sum_{j=1}^{n(l)} \alpha\left(e_{j, 1}^{(l)}\right) g_{l} \alpha\left(e_{1, j}^{(l)}\right), \quad$ and $\quad v=\sum_{l=1}^{m} \sum_{j=1}^{n(l)} \alpha\left(e_{j, 1}^{(l)}\right) v_{l} e_{1, j}^{(l)}$.

Then it is easily checked that $f$ and $g$ are projections, and that $v^{*} v=f$ and $v v^{*}=g$. So Condition (11) of Definition [5.1] holds with $f$ in place of $p_{1}$ and with $\alpha^{-1}(g)$ in place of $p_{2}$. We verify conditions (2) through (4). This will verify the hypotheses of Lemma [5.4] completing the proof.

For (2), let $a \in A$ and choose $b \in E$ such that $\|p a p-b\|<\varepsilon_{0}$. Then

$$
\|a-[b+(1-p) a(1-p)]\| \leq\|p a p-b\|+2\|p a-a p\|<3 \varepsilon_{0} .
$$


It is easily checked that $f$ commutes with every element of $E$, and $f$ commutes with $(1-p) a(1-p)$ because $f \leq p$, so $\|f a-a f\|<6 \varepsilon_{0} \leq \varepsilon$. Similarly, we get $\left\|\alpha^{-1}(g) a-a \alpha^{-1}(g)\right\|<\varepsilon$.

For (3), let $1 \leq l \leq m$ and $1 \leq j, k \leq n(l)$. Then

$$
\begin{aligned}
v f e_{j, k}^{(l)} f v^{*} & =\left[\alpha\left(e_{j, 1}^{(l)}\right) v_{l} e_{1, j}^{(l)}\right]\left[e_{j, 1}^{(l)} f_{l} e_{1, j}^{(l)}\right] e_{j, k}^{(l)}\left[e_{k, 1}^{(l)} f_{l} e_{1, k}^{(l)}\right]\left[e_{k, 1}^{(l)} v_{l}^{*} \alpha\left(e_{1, k}^{(l)}\right)\right] \\
& =\alpha\left(e_{j, 1}^{(l)}\right) v_{l} f_{l} v_{l}^{*} \alpha\left(e_{1, k}^{(l)}\right)=g \alpha\left(e_{j, k}^{(l)}\right) g=\alpha\left(\alpha^{-1}(g) e_{j, k}^{(l)} \alpha^{-1}(g)\right) .
\end{aligned}
$$

Since this is true for all $l, j$, and $k$, it follows that $v f b f v^{*}=\alpha\left(\alpha^{-1}(g) b \alpha^{-1}(g)\right)$ for every $b \in E$. Now let $a \in A$ and choose $b \in E$ such that $\|p a p-b\|<\varepsilon_{0}$. Then, using $f \leq p$ and $g \leq \alpha(p)$, we get

$$
\left\|v f a f v^{*}-\alpha\left(\alpha^{-1}(g) a \alpha^{-1}(g)\right)\right\| \leq\|f a f-f b f\|+\|g \alpha(a) g-g \alpha(b) g\|<2 \varepsilon_{0} \leq \varepsilon,
$$

as desired.

Finally, we verify (4). We have

$$
1-f=1-p+\sum_{l=1}^{m} \sum_{j=1}^{n(l)}\left(e_{j, j}^{(l)}-e_{j, 1}^{(l)} f_{l} e_{1, j}^{(l)}\right) \precsim r_{0}+\sum_{l=1}^{m} \sum_{j=1}^{n(l)} s_{l, j} \leq r,
$$

and similarly

$$
1-\alpha^{-1}(g)=1-p+\sum_{l=1}^{m} \sum_{j=1}^{n(l)}\left(e_{j, j}^{(l)}-e_{j, 1}^{(l)} \alpha^{-1}\left(g_{l}\right) e_{1, j}^{(l)}\right) \precsim r_{0}+\sum_{l=1}^{m} \sum_{j=1}^{n(l)} \alpha^{-1}\left(s_{l, j}\right) \precsim r .
$$

This completes the proof of (4), and of the theorem.

When the automorphism has finite order, we get strong tracial approximate innerness. Again, we need a lemma.

Lemma 6.5. Let $A$ be a simple unital $\mathrm{C}^{*}$-algebra with real rank zero, with stable rank one, and such that the order on projections over $A$ is determined by traces (Definition 2.4). Let $\alpha \in \operatorname{Aut}(A)$ be an automorphism such that $\alpha_{*}: K_{0}(A) \rightarrow$ $K_{0}(A)$ has finite order and such that $\alpha_{*}(\eta)-\eta$ is infinitesimal for every $\eta \in K_{0}(A)$. Let $p \in A$ be a projection. Then for every nonzero positive element $x \in A$ there exists a projection $p_{0} \leq p$ such that $\alpha\left(p_{0}\right) \sim p_{0}$ and such that $p-p_{0}$ is Murray-von Neumann equivalent to a projection in $\overline{x A x}$.

Proof. Without loss of generality $p \neq 0$. By Lemma 1.9 there is a nonzero projection $r \in \overline{x A x}$ such that $r \precsim p$. Set $\varepsilon=\inf _{\tau \in T(A)} \tau(r)>0$. Choose $N \in \mathbb{N}$ with $\frac{1}{N}<\frac{1}{2} \varepsilon$. Let $n$ be the order of $\alpha_{*}$. Use Lemma 2.3 of [19] to find projections $f, q_{0}, q_{1}, \ldots, q_{n N} \in A$ such that

$$
f+\sum_{k=0}^{n N} q_{k}=p \quad \text { and } \quad f \precsim q_{0} \sim q_{1} \sim \cdots \sim q_{n N} .
$$

For every $\tau \in T(A)$ and $\eta \in K_{0}(A)$, we have $-\tau_{*}\left(\left[1_{A}\right]\right) \leq n \tau_{*}\left(\alpha_{*}(\eta)-\eta\right) \leq$ $\tau_{*}\left(\left[1_{A}\right]\right)$ for all $n \in \mathbb{N}$. Therefore $\tau_{*} \circ \alpha_{*}=\tau_{*}$. Set

$$
\eta=\sum_{k=1}^{n N}\left[\alpha^{k}\left(q_{k}\right)\right]
$$


Then $\alpha_{*}(\eta)=\eta$ and $\tau_{*}(\eta)=n N \tau\left(q_{1}\right)<\tau(p)$. (We get strict inequality because $f+q_{0} \neq 0$.) Since the order on projections is determined by traces, there exists a projection $p_{0} \leq p$ such that $\left[p_{0}\right]=\eta$. Moreover, since $A$ has stable rank one, $\alpha\left(p_{0}\right) \sim p_{0}$.

For every $\tau \in T(A)$, we have

$$
(n N+1) \tau\left(q_{1}\right) \leq \tau(p) \leq 1 \quad \text { and } \quad \tau(f)+\tau\left(q_{0}\right) \leq 2 \tau\left(q_{1}\right)
$$

So

$$
\tau\left(p-p_{0}\right)=\tau(f)+\tau\left(q_{0}\right) \leq \frac{2}{n N+1}<\varepsilon \leq \tau(r) .
$$

It follows that $p-p_{0} \precsim r$.

Theorem 6.6. Let $A$ be an infinite dimensional simple separable unital $\mathrm{C}^{*}$-algebra with tracial rank zero. Let $\alpha \in \operatorname{Aut}(A)$ be an automorphism of finite order such that $\alpha_{*}(\eta)-\eta$ is infinitesimal for every $\eta \in K_{0}(A)$. Then $\alpha$ is strongly tracially approximately inner.

Proof. The proof is similar to that of Theorem 6.4 Let $F \subset A$ be finite, let $\varepsilon>0$, and let $x \in A$ be a positive element with $\|x\|=1$. Let $\varepsilon_{0}, r, r_{0}, p$, and $E=\bigoplus_{l=1}^{m} E_{l}$ with $E_{l} \cong M_{n(l)}$ and matrix units $\left(e_{j, k}^{(l)}\right)_{1 \leq j, k \leq n(l)}$ be as there, and further let $r_{l, j}$ and $s_{l, j}$ be as there. For $1 \leq l \leq m$ use Lemma 6.5 and Theorem 2.5 to find projections $f_{l} \leq e_{1,1}^{(l)}$ and partial isometries $v_{l} \in A$, such that

$$
v_{l}^{*} v_{l}=f_{l}, \quad v_{l} v_{l}^{*}=\alpha\left(f_{l}\right), \quad \text { and } \quad e_{1,1}^{(l)}-f_{l} \precsim s_{l, 1} .
$$

Define

$$
f=\sum_{l=1}^{m} \sum_{j=1}^{n(l)} e_{j, 1}^{(l)} f_{l} e_{1, j}^{(l)} \quad \text { and } \quad v=\sum_{l=1}^{m} \sum_{j=1}^{n(l)} \alpha\left(e_{j, 1}^{(l)}\right) v_{l} e_{1, j}^{(l)} .
$$

Then it is easily checked that $f$ is a projection, and that $v^{*} v=f$ and $v v^{*}=\alpha(f)$. So Condition (11) of Definition 4.2 holds with $f$ in place of $e$. The rest of the proof is essentially the same as the rest of the proof of Theorem 6.4 using Lemma 4.4 in place of Lemma 5.4

In Theorem 6.6. the hypothesis that $\alpha$ have finite order may be replaced by the assumption that $\alpha_{*}: K_{0}(A) \rightarrow K_{0}(A)$ have finite order, or by the assumption that the fixed points of $\alpha_{*}$ on $K_{0}(A)$ have dense image in the real affine functions on $T(A)$. One might hope to weaken it to the requirement that for every nonzero positive element $x \in A$, there be a nonzero projection $p \in \overline{x A x}$ such that $\alpha_{*}([p])=$ $[p]$. We do not know if this is possible.

\section{REFERENCES}

[1] C. A. Akemann and F. Shultz, Perfect $C^{*}$-algebras, Memoirs Amer. Math. Soc., vol. 55 no. 326(1985).

[2] B. Blackadar, A simple unital projectionless $C^{*}$-algebra, J. Operator Theory 5(1981), 63-71.

[3] B. Blackadar, Comparison theory for simple $C^{*}$-algebras, pages 21-54 in: Operator Algebras and Applications, D. E. Evans and M. Takesaki (eds.) (London Math. Soc. Lecture Notes Series no. 135), Cambridge University Press, Cambridge, New York, 1988.

[4] O. Bratteli, Inductive limits of finite dimensional $C^{*}$-algebras, Trans. Amer. Math. Soc. 171(1972), 195-234.

[5] J. Cuntz, The structure of multiplication and addition in simple $C^{*}$-algebras, Math. Scand. 40(1977), 215-233.

[6] J. Cuntz, K-theory for certain $C^{*}$-algebras, Ann. Math. 113(1981), 181-197. 
[7] S. Echterhoff, W. Lück, and N. C. Phillips, and S. Walters, The structure of crossed products of irrational rotation algebras by finite subgroups of $\mathrm{SL}_{2}(\mathbb{Z})$, preprint.

[8] T. Giordano, I. F. Putnam, and C. F. Skau, Topological orbit equivalence and $C^{*}$-crossed products, J. reine angew. Math. 469(1995), 51-111.

[9] K. R. Goodearl, Partially Ordered Abelian Groups with Interpolation, Math. Surveys and Monographs no. 20, Amer. Math. Soc., Providence RI, 1986.

[10] M. Izumi, Finite group actions on $C^{*}$-algebras with the Rohlin property. I, Duke Math. J. 122(2004), 233-280.

[11] M. Izumi, Finite group actions on $C^{*}$-algebras with the Rohlin property. II, Adv. Math. 184(2004), 119-160.

[12] J. A. Jeong and H. Osaka, Extremally rich $C^{*}$-crossed products and the cancellation property, J. Austral. Math. Soc. (Series A) 64(1998), 285-301.

[13] A. Kishimoto, Outer automorphisms and reduced crossed products of simple $C^{*}$-algebras, Commun. Math. Phys. 81(1981), 429-435.

[14] H. Lin, Tracially AF $C^{*}$-algebras, Trans. Amer. Math. Soc. 353(2001), 693-722.

[15] H. Lin, The tracial topological rank of $C^{*}$-algebras, Proc. London Math. Soc. 83(2001), 199234.

[16] H. Lin, An Introduction to the Classification of Amenable $C^{*}$-algebras, World Scientific, River Edge NJ, 2001.

[17] H. Lin, Classification of simple $C^{*}$-algebras with tracial topological rank zero, Duke Math. J. 125(2005), 91-119.

[18] T. A. Loring, Lifting Solutions to Perturbing Problems in $C^{*}$-Algebras, Fields Institute Monographs no. 8, American Mathematical Society, Providence RI, 1997.

[19] H. Osaka and N. C. Phillips, Stable and real rank for crossed products by automorphisms with the tracial Rokhlin property, Ergod. Th. Dynam. Sys., to appear (arXiv: math.OA/0409168).

[20] H. Osaka and N. C. Phillips, Furstenberg transformations on irrational rotation algebras, Ergod. Th. Dynam. Sys., to appear (arXiv: math.OA/0409169).

[21] H. Osaka and N. C. Phillips, Crossed products of simple $C^{*}$-algebras with tracial rank one by actions with the tracial Rokhlin property, in preparation.

[22] N. C. Phillips, Crossed products by finite cyclic group actions with the tracial Rokhlin property, unpublished preprint (arXiv: math.OA/0306410).

[23] N. C. Phillips, Every simple higher dimensional noncommutative torus is an AT algebra, preprint.

[24] N. C. Phillips, Finite cyclic group actions with the tracial Rokhlin property, preprint.

[25] M. A. Rieffel, Actions of finite groups on $C^{*}$-algebras, Math. Scand. 47(1980), 157-176.

[26] J. Rosenberg, Appendix to O. Bratteli's paper on "Crossed products of UHF algebras", Duke Math. J. 46(1979), 25-26.

[27] H. Takai, On a duality for crossed products of $C^{*}$-algebras, J. Funct. Anal. 19(1975), 25-39.

Department of Mathematics, University of Oregon, Eugene OR 97403-1222, USA.

E-mail address: ncp@darkwing.uoregon.edu 